
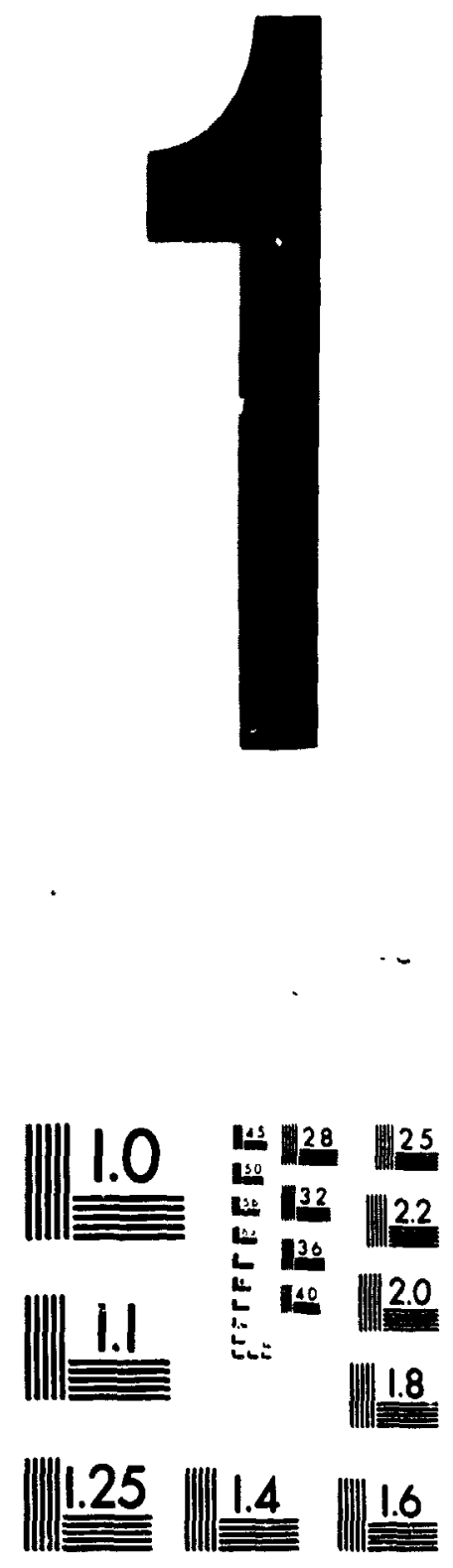

MICROCOPY RESOLUTION TFST CHART

NATIONAL BUAEAU OF STANDARDS

SIANDARO REFERENCE MATERIAL 1010

(ANSI and ISO TEST CHART NO ?) 
The quality of this microform is heavily dependent upon the quality of the original thesis submitted for microtilming. Every effort has been made to ensure the highest quality of reproduction possible.

If pages are missing. contact the university which granted the degree.

Some pages may have indistinct print especially if the original pages were typed with a poor typewriter ribbon or if the university sent us an interior photocopy.

Reproduction in full or in part of this microform is governed by the Canadian Copyright ACl, R.S.C. 1970. C. C-30, and subsequent amendments.
La reproduction, méme partielle, de celte microtorme est soumise à la Loi canadienne sur le droit dauteur. SRC 1970. c. C-30, et ses amendements subséquents. 


\title{
Background Acoustic Noise Suppression In Mobile Telephony
}

\author{
by \\ Robert Hebert, B. Eng. (Electrical)
}

A thesis submitted to the

Faculty of Graduate Studies and Research

in partial fulfillment of the requirements

for the degree of

Master of Engineering

Ottawa-Carleton Institute for Electrical Engineering

Faculty of Engineering

Department of Systems and Computer Engineering

Carleton University

Ottawa, Ontario

Canada

(C) Robert Hebert, 1990 
Canadian Theses Service Service des thèses canadiennes

The author has granted an irrevocable nonexclusive licence allowing the National Library of Canada to reproduce, loan, distribute or sell copies of his/her thesis by any means and in any form or format, making this thesis available to interested persons.

The author retains ownership of the copyright in his/her thesis. Neither the thesis nor substantial ex.c.cts from it may be printed or otherwise reproduced without his/her per. mission.
L'auteur a accordé une licence irrévocable et non exclusive permettant à la Bibliothéque nationale du Canada de reproduire, prêter. distribuer ou vendre des copies de sa these de quelque manière et sous quelque forme que ce soit pour mettre des exemplaires de cette thèse à la disposition des personnes intéressées.

L.'auteur conserve la propirèté du droit d'auteur qui protège sa thèse. Ni la thèse ni des extraits substantiels de celle-ci ne doivent être imprimés ou autrement reproduits sans son autorisation.

\section{ISBN $0-315-68883-1$}


The undersigned recommend to the Faculty of Graduate Studies and Research acceptance of the thesis

\author{
"Background Acoustic Noise \\ Suppression In Mobile Telephony"
}

\author{
submitted by \\ Robert Hebert, \\ Bachelor of Engineering (Electrical) \\ in partial fulfillment of the requirements \\ for the degree of Master of Engineering
}

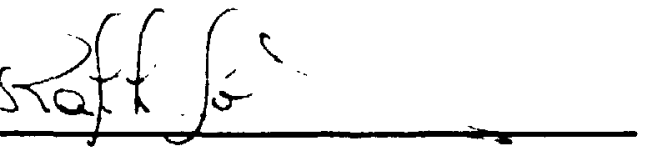

Thesis Supervisor, Department of Systems and Computer Engineering

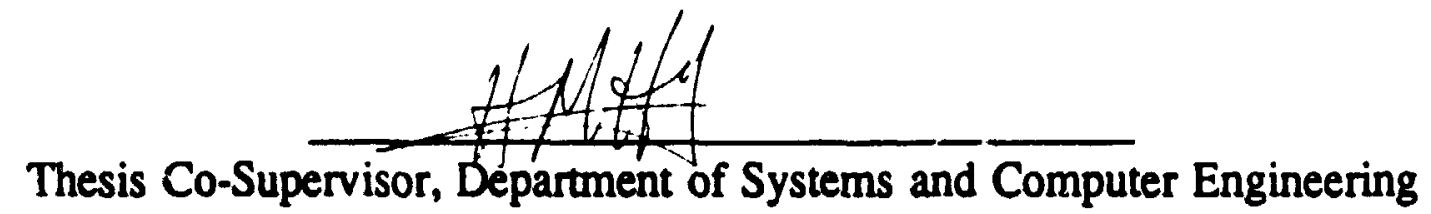

Chairman, Department of Systerns and Computer Engineering 


\section{Abstract}

This thesis investigates the background acoustic noise suppression in mobite telephony. Experimental background acoustic noise is obtained using a medium size car. Various driving conditions representing the normal use of a medium size car provides a good variety of noise samples for an in depth analysis. A wide variety of samples are obtained by strategically locating each microphone in the car. The problem's solution aims at analyzing a hands free telephony system where the speaker is away from the microphone.

A qualitative noise model is developed for ease of system's optimization. Based on the model, an in depth noise analysis is carried through in both the time and frequency domain. It shows under specific driving conditions how the various locations interact with the speaker's microphone (primary).

As a result of the background acoustic noise analysis, a 2 microphone adaptive noise canceller (ANC) is inuoduced using sub-band adaptation. Offering at least $11 \mathrm{~dB}$ signal to noise ratio (SNR) impiovement for all driving conditions. This one solves the "part band" noise enhancement problem associated with existing ANC. In light of these results, the investigation towards a more efficient ANC structure goes further. Two 4 microphone ANC structures are proposed using a beamformer (BF) like approach. Each respective structure offers $12 \mathrm{dF}$ and $15 \mathrm{~dB}$ SNR improvement without yielding any part band noise enhancement. Concluding results are obtained from experimental car background acoustic noise throughout the thesis. These emphasize the validity and the efficiency of the proposed structures. 


\section{Acknowledgments}

I would like to express some special thanks to professor R.A. Goubran, my thesis supervisor, for all his kindness and constant effort in providing me with the proper guidance throughout the thesis. By introducing me to the world of publication, he gave the research a constant flavour of challenge.

I would like to thank professor H.M. Hafez, my thesis co-supervisor, for his help and support throughout my research. His guidance toward ways of expressing research work were of great use in the thesis.

I would also like to thank Rob Mazoline and Bruce Wallace for the help they gave $\mathrm{m}:$ in reviewing my texts. 


\section{Table of Contents}

Abstract $\ldots \ldots \ldots \ldots \ldots \ldots \ldots \ldots \ldots \ldots \ldots \ldots \ldots \ldots$

Acknowledgments .......................... iv

List of Tables . . . . . . . . . . . . . . . . . . . . . vii

List of Figures $\ldots \ldots \ldots \ldots \ldots \ldots \ldots$. . . . . . . . . . . . . . . . . .

List of Acronyms ........................ xii

Chapter I Introduction . . . . . . . . . . . . . . . . . . . I

1.1 Background. . . . . . . . . . . . . . . . . . 1

1.2 Proposed Solutions. . . . . . . . . . . . . . . . . . . .

1.2.1 Types of Microphone Used. . . . . . . . . . . . . .

1.2.2 Speech Estimation. . . . . . . . . . . . . . . 2

1.2.3 Noist Estimation. . . . . . . . . . . . . . . . 3

1.2.3.1 Single Microphone. . . . . . . . . . . . . . . . . 3

1.2.3.2 Use of Multiple Microphones For Uncorrelated Noise Removal. . . 4

1.2.3.3 Use of Multiple Microphone For Correlated Noise Removal. . . . . 4

1.3 Thesis' Aim . . . . . . . . . . . . . . . . . . 6

1.4 Thesis Organization $\ldots \ldots \ldots \ldots \ldots \ldots \ldots$

Chapter 2 Noise Model . . . . . . . . . . . . . . . . . . . . 13

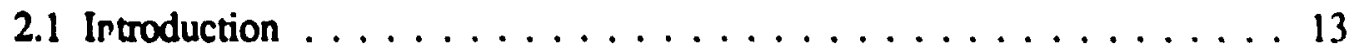

2.2 Type of Noise Sources and Their Properties. . . . . . . . . . . . . . 14

2.3 Generalized Noise Model . . . . . . . . . . . . . . . . . 15

2.4 Model Constraints for Successful Adaptive Noise Cancellation . . . . . 26

Chapter 3 Recording set up and Testing . . . . . . . . . . . . . 28

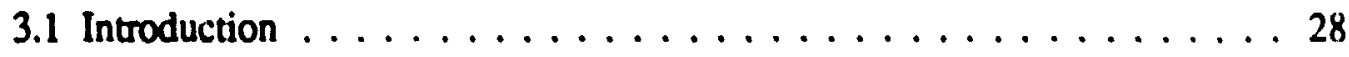

3.2 Microphone Locations . . . . . . . . . . . . . . . 28

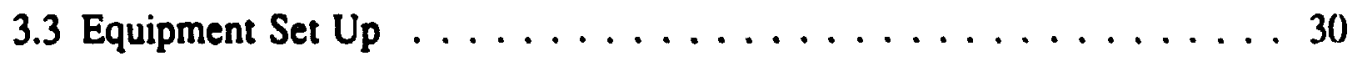

3.4 Measurement Set Up Validation $\ldots \ldots \ldots \ldots$.................. 31

3.5 Driving Conditions . . . . . . . . . . . . . . . . 32 
Chapter 4 Data Analysis . . . . . . . . . . . . . . . . . 35

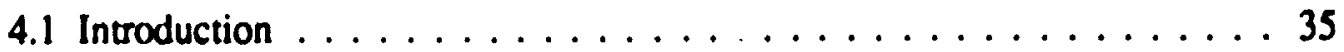

4.2 Power Spectral Density . . . . . . . . . . . . . . . . . . . 35

4.3 Correlation . . . . . . . . . . . . . . . . . . . . . . . 39

4.3.1 Autocorrelation . . . . . . . . . . . . . . . 41

4.3.2 Cross-Correlation . . . . . . . . . . . . . . 41

Chapter 5 Two Microphone Technique for Noise Cancellation . . . . . . 47

5.1 Introduction . . . . . . . . . . . . . . . . 47

5.2 Transfer Function Estimation . . . . . . . . . . . . . . 48

5.3 Algorithm ......................... 56

5.4 Results Of a Two Microphone ANC . . . . . . . . . . . . 58

5.5 Sub-Band Adaptation . . . . . . . . . . . . . . . . . 67

5.6 Results of Sub-Band Adaptation . . . . . . . . . . . . 68

5.6.1 Lower Band Adaptation . . . . . . . . . . . . . . . . . 68

5.6 .2 Upper Band Adaptation . . . . . . . . . . . . . . . . 69

5.6.3 Sub-Band Adaptation Over Both Band . . . . . . . . . . . . 72

Chapter 6 Parallel Adaptation ................... 83

6.1 Introduction . . . . . . . . . . . . . . . . 83

6.2 General Beamformer Approach . . . . . . . . . . . . . . 83

6.2.1 Temporal Filtering . . . . . . . . . . . . . . . 84

6.2.2 Spatial Filtering . . . . . . . . . . . . . . 85

6.3 Use Of Constraint For Algorithm Optimization . . . . . . . . . . . . 89

6.44 Microphone Adaptive Noise Cancellation and Results . . . . . . . . . . 90

6.4.1 2 Level Adaptation System and Results . . . . . . . . . . . . 91

6.4 .2 Sequential Approach. . . . . . . . . . . . . . . . . 94

Chapter 7 Conciusiun ...................... 100

7.1 Discussion on the Results . . . . . . . . . . . . . . 100

7.2 Areas of Further Research . . . . . . . . . . . . . . . . 101

References ............................... 103 


\section{List of Tables}

Table 3.1 Driving Conditions During Field Tests . . . . . . . . . . . . 33

Table 3.2 Recording Level During Field Tests . . . . . . . . . . . . . . 34 


\section{List of Figures}

Figure 1.1 Major Sources of Background Acoustic Noise in a Car . . . . . . . . 1

Figure 1.2 Noise Canceller Using an Array of Microphones . . . . . . . . . . 5

Figure 1.3 Adaptive Noise Cá,celler Using Correlated Noise . . . . . . . . . . . 6

Figure 1.4 Adaptation Using a Two Microphone ANC; $\ldots \ldots \ldots \ldots \ldots$

Figure 1.5 Lower Band Cross-Correlation Between Location 2 and the Primary L.xation (at the Steering Wheel) $\ldots \ldots \ldots \ldots$

Figure 1.6 Upper Band Cross-Correlation Between Location \# 2 and the Primary

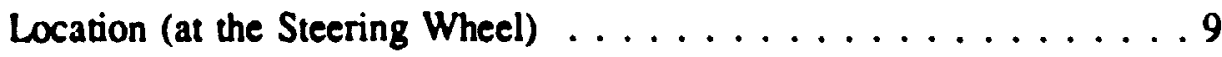

Figure 1.7 Lower Band Adaptation Using a 2 Microphone ANC; . . . . . . . 10

Figure 1.8 Uprer Band Adaptation Using a 2 Microphone ANC; . . . . . . . 11

Figure 1.9 Lower and Upper Band Adaptation Using a 2 Microphone ANC; . . 12

Figure 2.1 Transfer Functions Existing Between 2 Locations . . . . . . . 14

Figure 2.2 Localized Noise Configuration . . . . . . . . . . . . . 15

Figure 2.3 Distributed Noise Configuration . . . . . . . . . . . . 16

Figure 2.4 Multiple Input Multiple Output Noise System Configuration . . . . . 17

Figure 3.1 Microphone Disposition During Field Tests . . . . . . . . . . . . . 30

Figure 3.2 Recording Set-Up Configuration . . . . . . . . . . . . 31

Figure 4.1 Power Spectrum Density, Car Idling, Fan Off . . . . . . . . . . . . 37

Figure 4.2 Power Spectrum Density, Engine Off, Fan On . . . . . . . . 38

Figure 4.3 Power Spectrum Density, Car Speed $60 \mathrm{Km} / \mathrm{hr}$, Fan On . . . . . . . . 39

Figure 4.4 Power Spectrum Density, Car Speed $100 \mathrm{Km} / \mathrm{hr}$, Fan On . . . . . . 40 
Figure 4.5 Autocorrelation of the Lower Band (below 1.5 KHz), Engine Off. Fian On ........................ tz

Figure 4.6 Autocorrelation of the Upper Band (above 1.5 KHz), Engine Off, lian On ..........................

Figure 4.7 Cross-Correlation Of the Lower Band (below $1.5 \mathrm{KHz}$ ) With Respect w the Primary Microphone (at the Steering Wheel); Car Speed $60 \mathrm{Km} / \mathrm{hr}$.

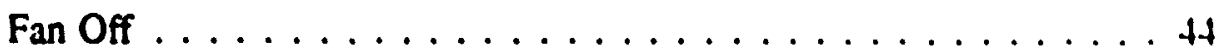

Figure 4.8 Cross-Correlation Of the Upper Band (above $1.5 \mathrm{KHz}$ ) With Respect it the Primary Microphone (at the Steering Wheel); Car Speed $60 \mathrm{Km} / \mathrm{hr}$. Fan Off $\ldots \ldots \ldots \ldots \ldots \ldots \ldots \ldots \ldots \ldots \ldots$

Figure 5.1 Transversal Filter With Variable Coefficient $\ldots \ldots \ldots \ldots \ldots \ldots$. . .

Figure 5.2 Mean Square Error Distribution With Respect to $W(t) \ldots \ldots \ldots$. . . 51

Figure 5.3 Two Microphone ANC Configuration $\ldots \ldots \ldots \ldots \ldots \ldots$. . . 59

Figure 5.4 Result Of a Two MicrophoneANC, Engine Off, Fan On; . . . . 6 60

Figure 5.5 Result Of a Two MicrophoneANC, Car Idling, Fan Off; $\ldots \ldots \ldots 61$

Figure 5.6 Result Of a Two MicrophoneANC, Car Idling, Fan On; . . . . 62

Figure 5.7 Result Of a Two MicrophoneANC, Car Speed $60 \mathrm{Km} / \mathrm{hr}$, 5an Off; . 63

Figure 5.8 Result Of a Two MicrophoneANC, Car Speed $60 \mathrm{Km} / \mathrm{hr}$, Fan On; . . 64

Figure 5.9 Result Of a Two MicrophoneANC, Car Speed $100 \mathrm{Km} / \mathrm{hr}$, Fan Off; . 65

Figure 5.10 Result Of a Two MicrophoneANC, Car Speed $100 \mathrm{Km} / \mathrm{hr}$, Fan On; . 66

Figure 5.11 Low-Pass and High-Pass Filtering $\ldots \ldots \ldots \ldots \ldots 67$

Figure 5.12 Sub-Band Two MicrophoneANC Configuration $\ldots \ldots \ldots \ldots \ldots 8$ 
Figure 5.13 Result Of a Two MicrophoneSub-Band ANC (lower band filtering), Car Idling, Fan Off; . . . . . . . . . . . . . . . . . . . 69

Figure 5.14 Result Of a Two MicrophoneSub-Band ANC (lower band filtering), Car Idling, Fan On; ..................... . . . .

Figure 5.15 Result Of a Two MicrophoneSub-Band ANC (lower band filtering), Car Speed $100 \mathrm{Km} / \mathrm{hr}$, Fan On; ................. 71

Figure 5.16 Result Of a Two MicrophoneSub-Band ANC (upper band filtering), Engine Off, Fan On; ................... . . . .

Figure 5.17 Result Of a Two MicrophoneSub-Band ANC (upper band filtering), Car Idling, Fan Off; . . . . . . . . . . . . . . . . . 74

Figure 5.18 Result Of a Two MicrophoneSub-Band ANC (upper band filtering), Car Speed $60 \mathrm{Km} / \mathrm{hr}$, Fan On; . . . . . . . . . . . . . . 75

Figure 5.19 Result Of a Two MicrophoneSub-Band ANC (upper and lower band independently filtered), Engine Off, Fan On; .......... 76

Figure 5.20 Result Of a Two MicrophoneSub-Band ANC (upper and lower band independently filtered), Car Idling, Fan Off; . . . . . . . . . 77

Figure 5.21 Result Of a Two MicrophoneSub-Band ANC (upper and lower band independently filtered), Car Idling, Fan On; . . . . . . . . . 78

Figure 5.22 Result Of a Two MicrophoneSub-Band ANC (upper and lower band independently filtered), Car Speed $60 \mathrm{Km} / \mathrm{hr}$, Fan Off; . . . . . . . 79

Figure 5.23 Result Of a Two MicrophoneSub-Band ANC (upper and lower band independently filtered), Car Speed $60 \mathrm{Km} / \mathrm{hr}$, Fan On; . . . . . . 80

Figure 5.24 Result Of a Two MicrophoneSub-Band ANC (upper and lower band independently filtered), Car Speed $100 \mathrm{Km} / \mathrm{hr}$, Fan Off; . . . . . 81 
Figure 5.25 Result Of a Two MicrophoneSub-Band ANC (upper and lower band independently filtered), Car Speed $100 \mathrm{Km} / \mathrm{hr}$, Fan On; Output: -+. . 82

Figure 6.1 Multiple Reference ANC Configuration $\ldots \ldots \ldots \ldots \ldots \ldots$. $\ldots \ldots$

Figure 6.2 Temporal Filtering in the Multiple Reference ANC . . . . . . 86

Figure 6.3 Spatial Filtering Configuration . . . . . . . . . . 87

Figure 6.4 Parallel Structure, 2 Level ANC Configuration . . . . . . . . . 91

Figure 6.5 Result Of a 4 Microphone Sub-Band ANC (lower band filtering), Car Idling, Fan Off; $\ldots \ldots \ldots \ldots \ldots \ldots \ldots \ldots$. $4 . \ldots \ldots$

Figure 6.6 Result Of a 4 Microphone Sub-Band ANC (lower band filtering), Car Speed $100 \mathrm{Km} / \mathrm{hr}$, Fan Off; $\ldots \ldots \ldots \ldots \ldots \ldots$

Figure 6.7 Parallel Adaptation, Sequential Structure . . . . . . . . . 96

Figure 6.8 Result Of a 4 Microphone Sub-Band ANC (lower band filtering), Car Idling, Fan Off; $\ldots \ldots \ldots \ldots \ldots \ldots \ldots \ldots \ldots$

Figure 6.9 Result Of a 4 Microphone Sub-Band ANC (lower band filtering), Car Speed $100 \mathrm{Km} / \mathrm{hr}$, Fan Off; . . . . . . . . . . . . . 99 


\title{
List of Acronyms
}

\author{
ANC : adaptive noise canceller \\ AD : analog to digital converter \\ BLS : block least square \\ BF : beamformer \\ $\mathrm{cm}$ : centimeter \\ $\mathrm{dB}$ : decibel \\ DC/AC : direct current to alternative current \\ ef : efficiency tactor \\ EM : estimate maximize \\ FIR : finite impulse response \\ $\mathrm{Hz}$ : hertz \\ K : kilo \\ $\mathrm{KHz}$ : kilohertz \\ KLT : Karhunen-Loeve Transform \\ $\mathrm{Km} / \mathrm{hr}$ : kilometer per hour \\ LMS : least mean square \\ LPC : linear predictive coder \\ LS : least square \\ mic : microphone \\ MIMO : multiple input multiple output \\ inmse : minimum mean square error \\ mse : mean square error \\ msec : millisecond \\ PSD : power spectrum density \\ RLS : recursive least square
}


SNR : signal to noise ratio 


\section{Chapter 1 Introduction}

\subsection{Background.}

In mobile telephony, background acoustic noise degrades the performance of voice communications. The major sources contributing to background acoustic noise are shown in figure 1.1. They consist of street noise, road noise, engine, fan motor, air flow, vibration and man made noise $[1,2]$.

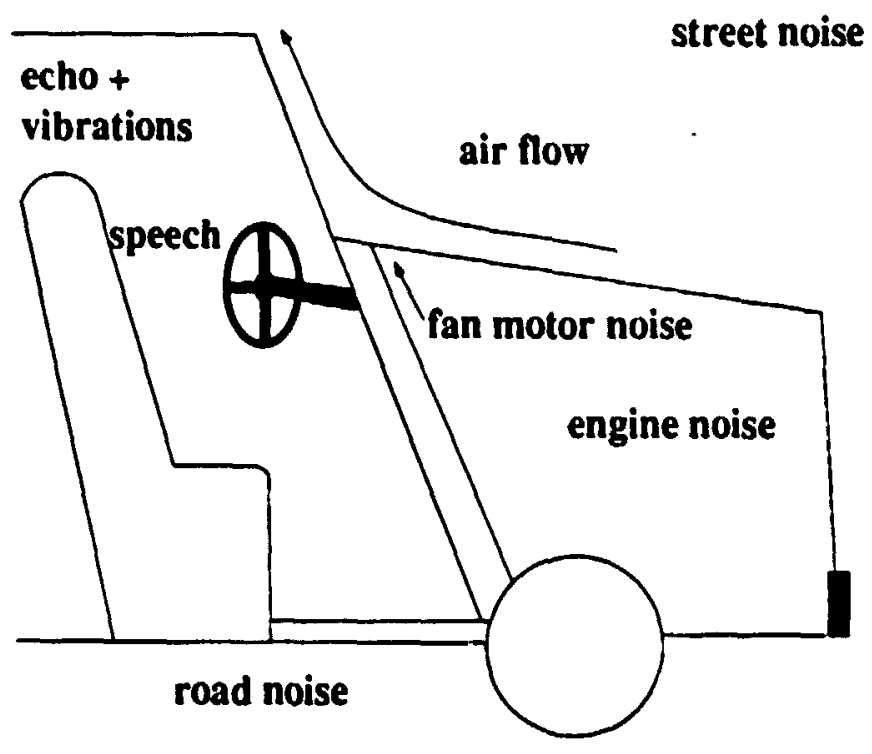

Figure 1.1 Major Sources of Background Acoustic Noise in a Car 
The background acoustic noise is also a major problem in voice/data integrated systems $[1,2,29,31]$. The problem is more serious in a hands-tree telephony system where the distance between the speaker and the microphone is relatively large $[2,20,26]$.

\subsection{Proposed Solutions.}

Many approaches have been proposed for reducing the background acoustic noise. A noise suppression system may include one or several of these methods. The most common solutions are described in the following subsections.

\subsubsection{Types of Microphone Used.}

In mobile telephony, background acoustic noise comes from all directions while the noise, the speech comes from a specific area. An omnidirectional microphone picks up the speech as well as most of the backgrnilnd acoustic noise. The use of unidirectional microphones limits the capture of background acoustic noise to the area to which the microphone is pointing. It has been shown that, in some cases the use of these microphones can provide up to $4.8 \mathrm{~dB}$ signal to noise ratio (SNR) improvement over the omnidirectional family [26].

\subsubsection{Speech Estimation.}

Given a noisy speech signal, one can discriminate between the speech and the noise by making use of a speech model. The model is based on the quasi stationarity property of speech. The aim is to extract the speech parameters from the corrupted signal using only one microphone. The system then attempts to reconstruct the speech signal free of noise. An example of this approach is the well known Linear Predictive Coder (LPC). In this case, the system first estimates the pitch period. Then it estimates the speech 
vocal tract characteristics $[3,8,13,14,18,64]$. This technique has shown a signal to noise ratio (SNR) improvement of up to $7 \mathrm{~dB}$ in a $0 \mathrm{~dB}$ environment [45].

This approach has proved suitable in removing the cocktail party effect [27]. In this case, the aim is to isolate one speaker out of many. This is done by evaluating the pitch of the desired person. Then, the speech parameters pertaining to the pitch of interest are extracted.

\subsubsection{Noise Estimation.}

Another approach is to make use of a noise model. The technique is composed of two steps. First, it estimates the noise, then subtract the estimated noise from the noisy signal. Many techniques stem from this approach. They can be divided in two categories:

(i) a single microphone system; and

(ii) a multiple microphones systems.

The following methods make use of the noise model:

1.2.3.1 Single Microphone. This method works only if the noise is stationary i.e. its characteristics do not vary with time. During speechless periods, the system evaluates the background acoustic noise. When the speech becomes active, the estimated noise is subtracted from the noisy speech. In most cases, the noise is composed of specific frequency components. The aim of the system is to find these frequency components. The system then places some nulls at their frequency locations.

As mentioned in [31], the problein associated with this technique is the result of an annoying musical tone effect. Obviously, this technique is not suitable for rapid time variant noise. Hence, it does not seem to be adequate for a mobile telephony system. 
1.2.3.2 Use of Multiple Microphones For Uncorrelated Noise Removal. It is a well known fact that there exist some uncorrelated background acoustic noise in a mobile telephony system. In [26], a solution for removing the uncorrelated nois: is proposed. A system consisting of an array of microphones disposed around the speech source is shown in figure 1.2. The concept is simple. By knowing the exact lociation of the speaker with respect tu each microphone, delays are insened at each microphone so that the various speech samples are added coherently. Since every noise sample is uncorrelated with one another, adding $\mathbf{N}$ samples ar.d dividing the results by $\mathbf{N}$ should average out the noise. By inspection, it is clear that this method tends to limit the noise level to its mean without affecting the speech. For a zero mean background acoustic noise process, the technique can be very effective. As indicated in [26], it provides some background noise attenuation and it seems efficient for reducing the uncorrelated noise. However, the problem lies in accurately estimating the speaker position with respect to each microphone.

\subsubsection{Use of Multiple Microphone For Correlated Noise Removal. Another ap-} proach makes use of the noise correlation. This approach is the back bone of this thesis research. It is discussed well in the literature $[2,4,26,69]$. Figure 1.3 shows the configuration process. This technique compares background acoustic noise $\left(\boldsymbol{n}_{\mathbf{r}}(t)\right)$ observed at the reference locations with that observed at the primary location $\left(n_{p}(t)\right)$. Using $n_{r}(t)$ it tries to estimate a transfer function $\underline{W}(t)$ which would best reproduce the noise at the primary $n_{p}(t)$. The transfer function $\underline{W}(t)$ is then optimized using the error $e(t)$ i.e. the difference between the actual noise at the primary $n_{p}(t)$ and its estimate $\tilde{n}_{p}(t$, .

Experimental results were obtained from tests performed on a medium size car $[2,69]$. In general, the ANC provides good noise attenuation in the lower end of the acoustic band to the detriment of the upper acoustic band $[2,26,69]$. Figure 1.4 shows 


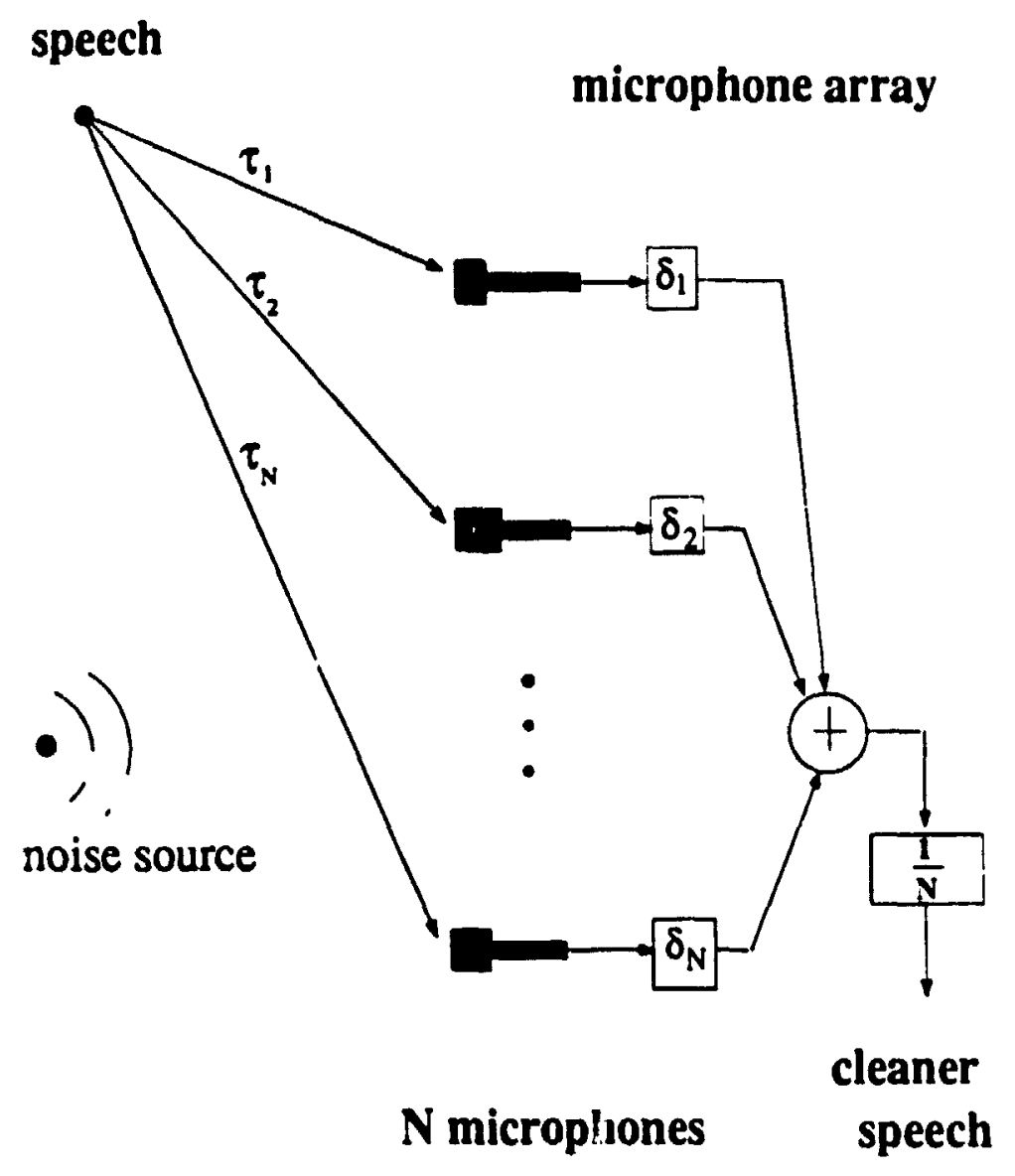

Figure 1.2 Noise Canceller Using an Array of Microphones

the result of a 2 microphone ANC. Note that the lower band noise (below $1.5 \mathrm{KHz}$ ) is suppressed considerably. However the upper band noise is enhanced. As mentioned earlier, this is true for every ANC used in mobile telephony $[2,26,69]$.

Sub-band adaptation partially overcome this problem $[2,28,69]$. The acoustic band is split in two bands: the lower (below $1.5 \mathrm{KHz}$ ) and the upper (above $1.5 \mathrm{KHz}$ ). Figures 1.5 and 1.6 show the existence of a certain degree of correlation between the two points 


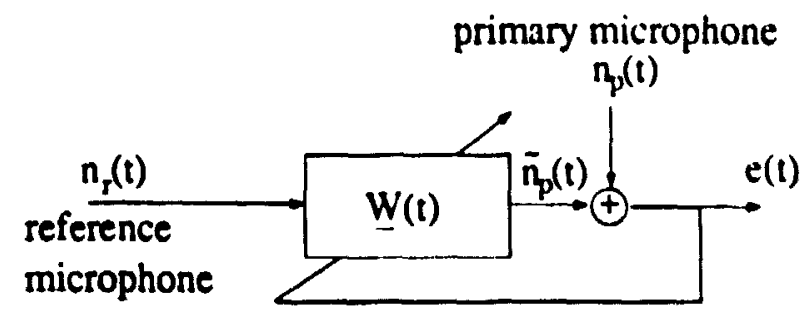

Figure 1.3 Adaptive Noise Canceller Using Correlated Noise

of observation. This indicates that adaptation can be performed independently over each band. Hence, two adaptive noise cancellers are needed, one for each band.

Figure 1.7 shows the result of lower band adaptation. Obviously good noise cancellation is achieved in the lower band without affecting the upper band. Figure 1.8 shows the result of upper band adaptation. Here, noise suppression takes place in the upper band without affecting the lower band. Figure 1.9 shows the combined effect of upper and lower band adaptation.

\subsection{Thesis' Aim}

The aim of the thesis is to investigate the use of two or more microphones for cancelling the background acoustic noise in a medium size car. This must be achieved without enhancing the background acoustic noise of any part of the acoustic band. The basic concept makes use of the correlated noise which exists between various locations in a car environment. 
Car Speed $60 \mathrm{Km} / \mathrm{hr}$, Fan Off, Microphone \# 2 (Under the Steering Wheel)

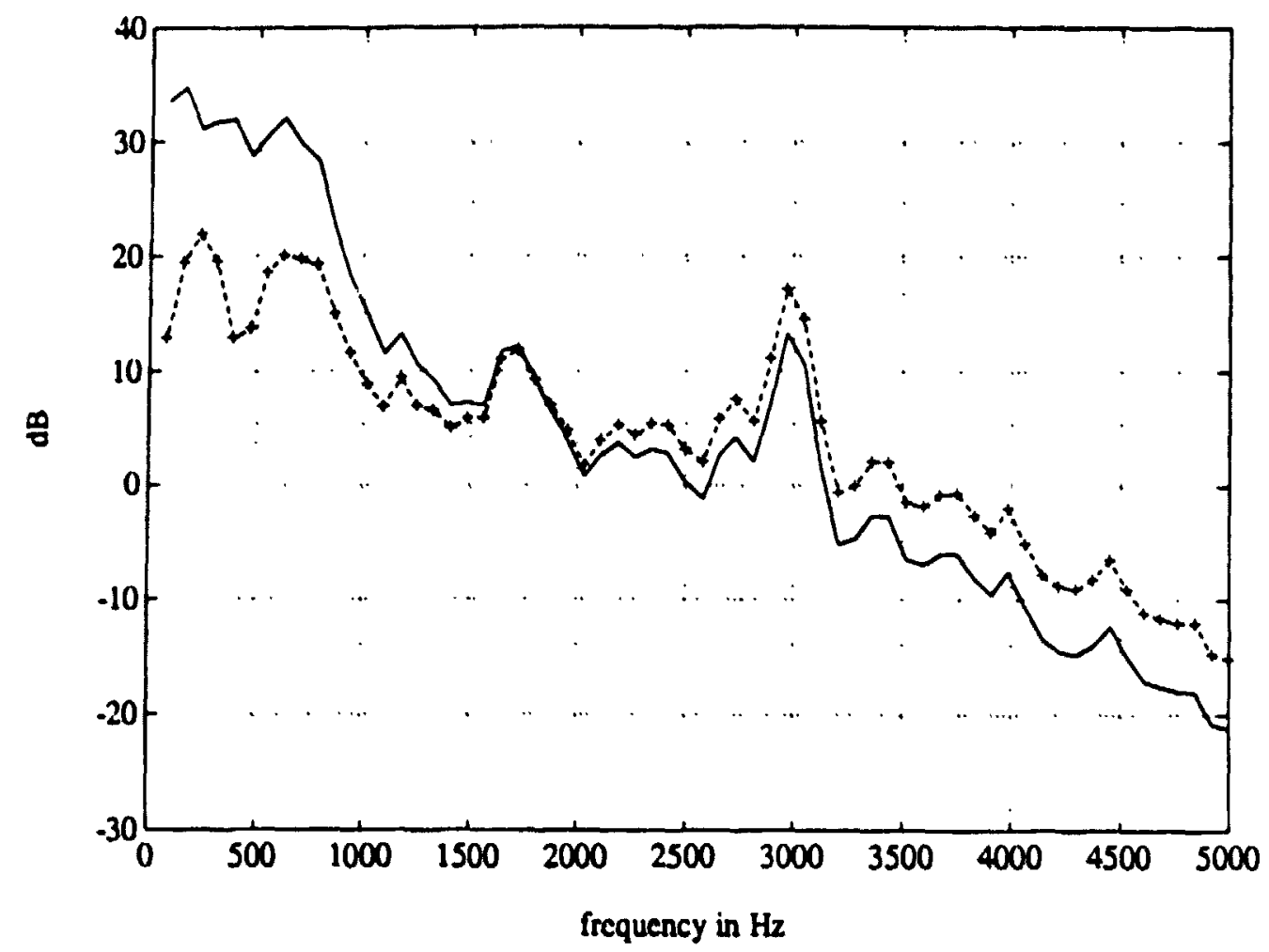

Figure 1.4 Adaptation Using a Two Microphone ANC; Filtered Output :---

\subsection{Thesis Organization}

The thesis is organized in 7 chapters. Chapter 2 proposes a qualitative noise model which theoretically supports any adaptive noise canceller (ANC) system. Chapter 3 covers the recording setup and testing conditions under which the data was acquired. Chapter 4 deals with the data analysis. Chapter 5 covers the use of a two microphone noise cancellation system. Chapter 6 proposes a parallel approach for the use of more than 2 microphone ANC. Some structures as well as their results are presented. Chapter 7 concludes with a discussion of the results, and further research. 
Car Speed $60 \mathrm{Km} / \mathrm{hr}$, Fan Off, Microphone \# 2 (Under the Steering Wheel)

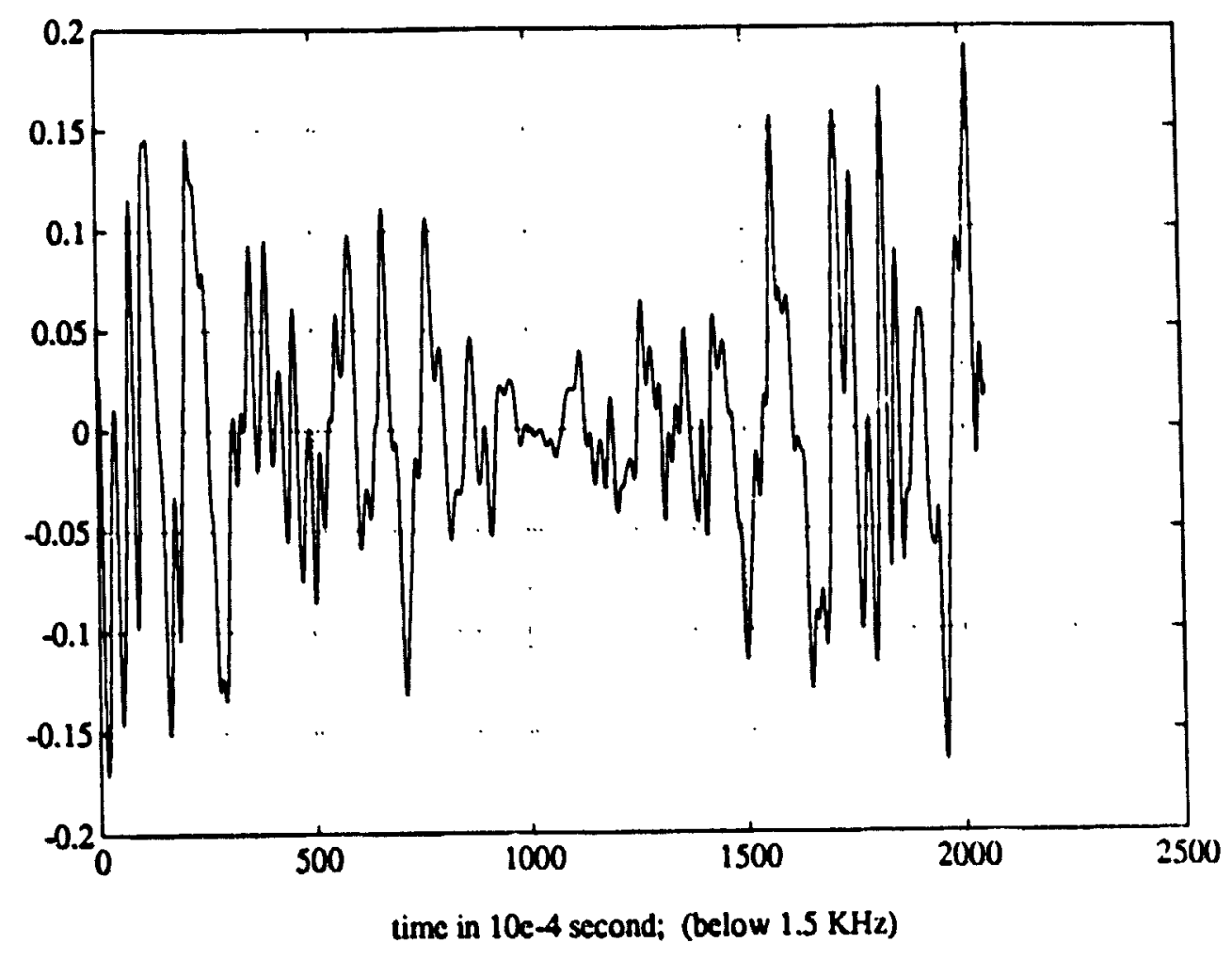

Figure 1.j Lower Band Cross-Correlation Between Location

\# 2 and the Primary Location (at the Steering Wheel) 
Car Speed $60 \mathrm{Km} / \mathrm{hr}$, Fan Off, Microphone \# 2 (Under the Steering Wheel)

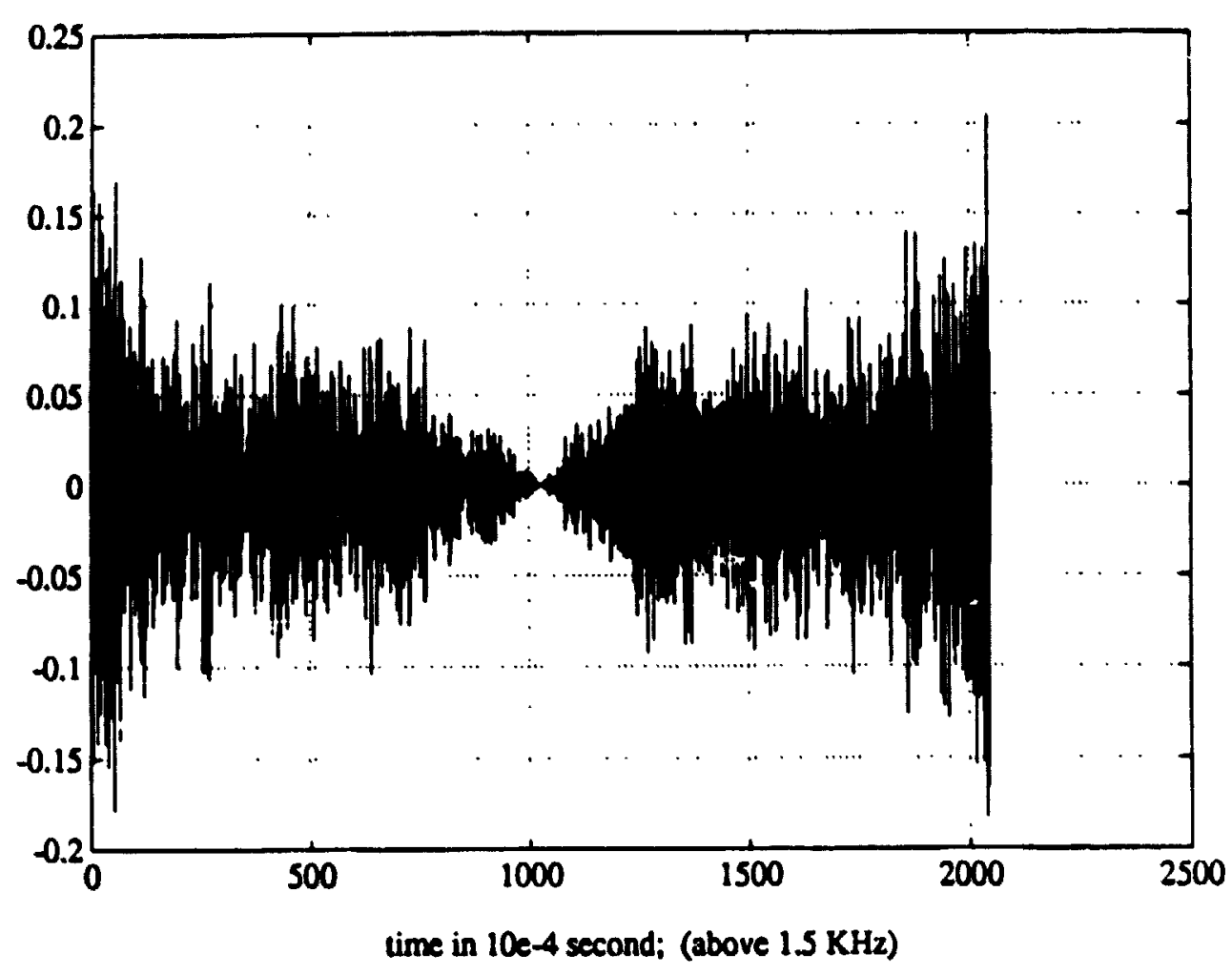

Figure 1.6 Upper Band Cross-Correlation Between Location

\# 2 and the Primary Location (at the Steering Wheel) 
Car Speed $60 \mathrm{Km} / \mathrm{hr}$, Fan Off, Microphone 2 (Under the Steering Wheel)

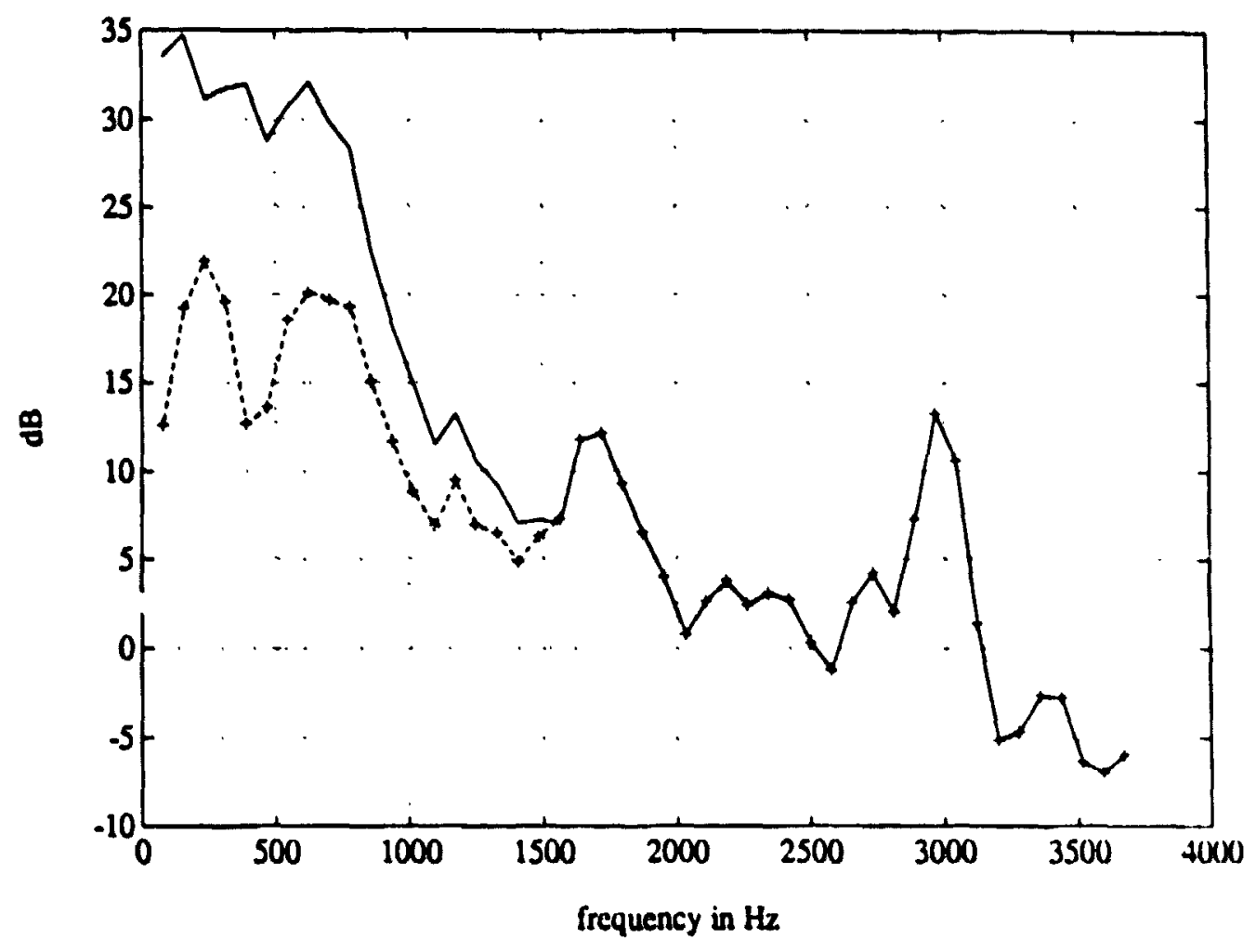

Figure 1.7 Lower Band Adaptation Using a 2 Microphone ANC; Filtered Output :--- 
Car Speed $60 \mathrm{Km} / \mathrm{hr}$, Fan Off, Microphone \# 2 (Under the Steering Wheel)

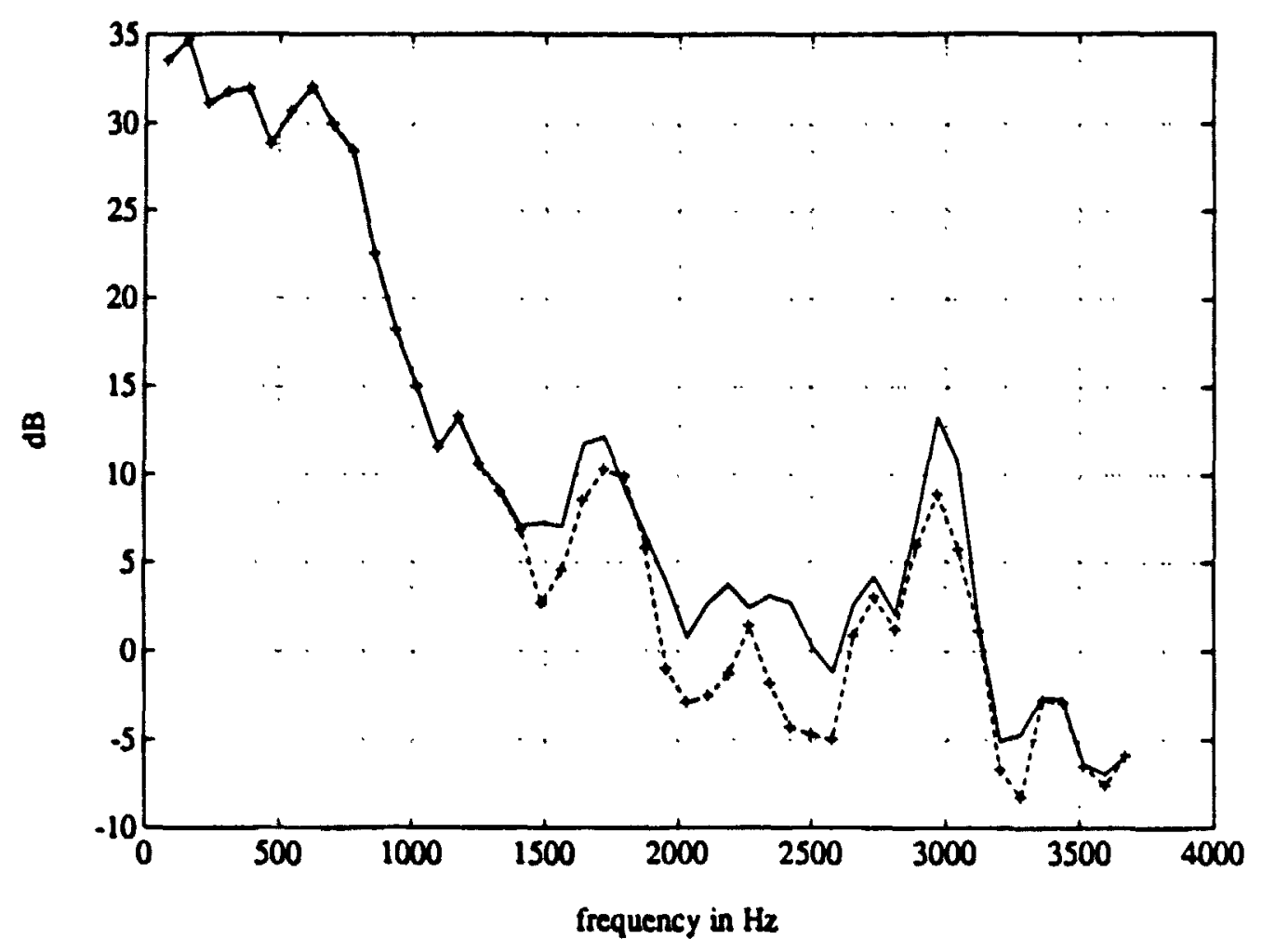

Figure 1.8 Upper Band Adaptation Using a 2 Microphone ANC; Filtered Output :-+- 
Car Speed $60 \mathrm{Km} / \mathrm{hr}$, Fan Off, Microphone \#2 (Under the Steering Wheel)

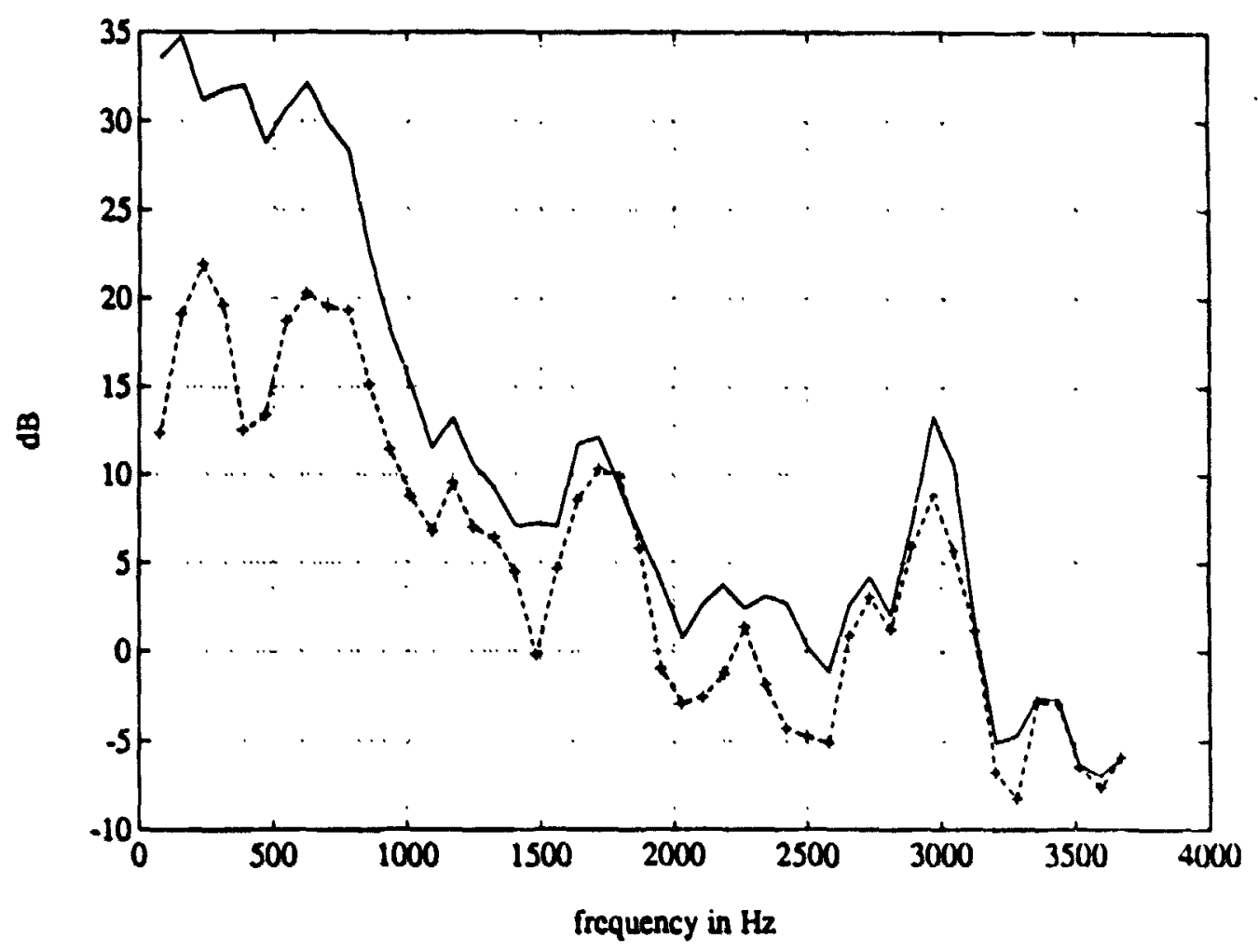

Figure 1.9 Lower and Upper Band Adaptation

Using a 2 Microphone ANC; Filtered Output :-t- 


\section{Chapter 2 Noise Model}

\subsection{Introduction}

Background acoustic noise in cars is composed of many noise source:s. The sources originate from outside and within the car cabin $[1,2,69]$. They can be classified as localized or distributed processes. The major outside noise processes comie from the air flow, the street, the engine, the road and, the fan motor. The interior noise come from the fan air blowing, the echo, the vibration and, the passenger.

Unfortunately, each noise process has particular characteristics (or properties) $[1,2,-0,21]$. Let's consider a noise source and two arbitrary ubservation points in the car. There are three transfer functions governing the propagation of noise from the source to the two observation point as shown in figure 2.1. They are as followed:

$A(z)$ : from the source to the primary observation point;

$H(x)$ : from the source to the secondary observation point; and

HA(z): from the secondary to the primary.

$\delta_{1}$ and $\delta_{2}$ are the propagation delays from the source to the primary and secondary point, respectively.

The model shown in figure ' 2.1 can be generalized to a multi-noise sources model; but, because of the noise characteristics complexity, the noise model can only be a 


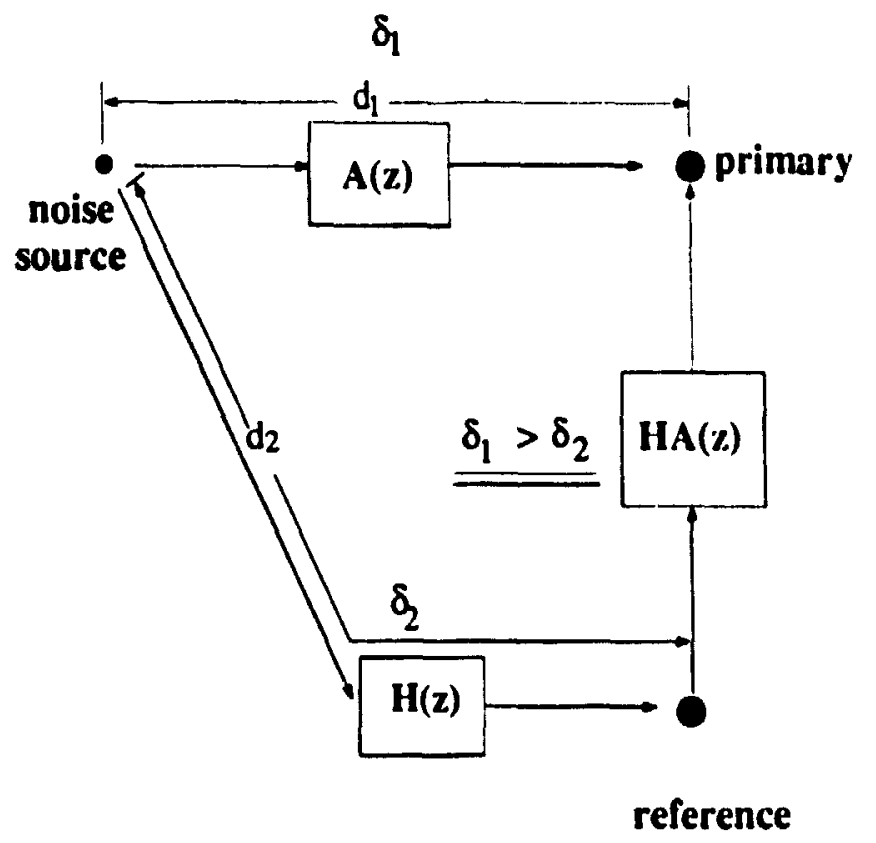

Figure 2.1 Transfer Functions Existing Between 2 Locations

qualitative representation of the actual system. However, it does provide some insights with regard to system's optimization.

This chapter is divided in 3 parts. The first part discusses the different types of noises and some of their properties. The second part establishes the concept of multi-noise processes interacting within the car system, and, the third part shows what minimum constraints on the model must be met in order for the noise cancellation to be feasible.

\subsection{Type of Noise Sources and Their Properties.}

The noise processes can be classified as being localized or distributed $[1,2,69]$. $A$ localized noise source is generated from a specific area. It can be seen as a point source. As shown in figure 2.2 a simple transfer function separates the noise source from an 


\section{localized noise}

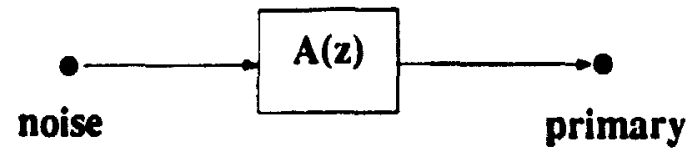

source

Figure 2.2 Localized Noise Configuration

observation point. This function depends on the distance and the media located between the two points.

A distributed noise source comes from a wide area. An example of this is the air flow on and around the car. As shown in figure 2.3, it can be represented by many transfer functions linking all the point sources to the observation point. This explains the complexity associated with this type of noise.

In general, the complexity of the transfer function linking two poinis increases with the distance and the type of material separating them. Therefore in a multi-noise source system (or in the presence of distributed noise), the microphones should be located close to each other for the model to be valid

\subsection{Generalized Noise Model}

In this section, the aim is to establish a general mathematical noise model which qualitatively represents tie car background acoustic noise. This model will then help in finding ways for optimizing any ANC. The proposed model consists of $k$ noise sources, $\mathrm{m}$ reference microphones, a speech source and a primary microphone as shown in figure $2.4[20,21]$. 


\section{distributed noise}

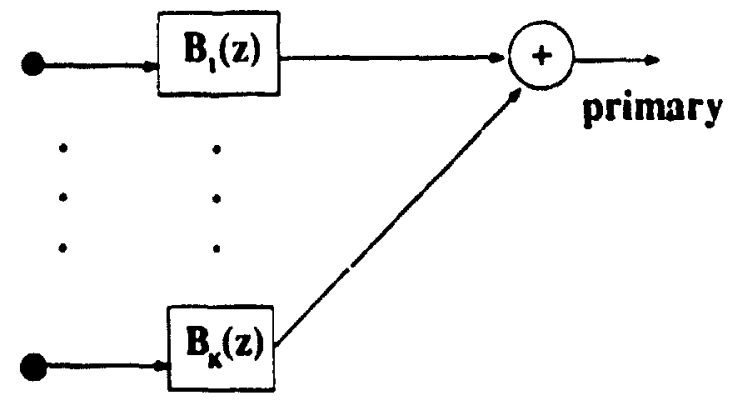

Figure 2.3 Distributed Noise Configuration

It is assumed that every transfer function can be approximated by a finite impulse responses (FIRs) of length $\mathbf{N}$. This allows the use of linear regression for estimating the transfer functions [58-62]. In figure 2.4., the speech goes through a transfer function $\underline{A}(t)$ before reaching the primary. Simultaneously, the speech goes through $m$ transfer functions $\left(\underline{B}_{1}(t) ; i=1\right.$ to $\left.m\right)$ prior to reaching the $m$ references. The $k$ noise sources go through $k$ transfer functions $C_{j}(t)$ prior to reaching the primary. These same $k$ noise sources go through $k$ different transfer function $\left.\underline{D}_{y}(t)\right)$ prior to reaching each of the $m$ references. At the primary $e(t)$ and $e_{j}(t)$ at the $j^{\text {th }}$ reference, are errors added which compensate for any unaccounted process and for the non-linearity part of the noise processes.

One must remember that the ANC aim is to estimate the noise at the primary using the observed noise at the reference microphones. As a logical step, the observed processes at the primary and at the references should be defined in term of the noise sources. Hence, as shown in figure 2.4. the primary output is composed of transformed speech and noise processes.

$$
x(t)=\underline{s}^{T}(t) \underline{A}(t)+\underline{n}^{T}(t) \underline{C}^{T}(t)+e_{x}(t)
$$




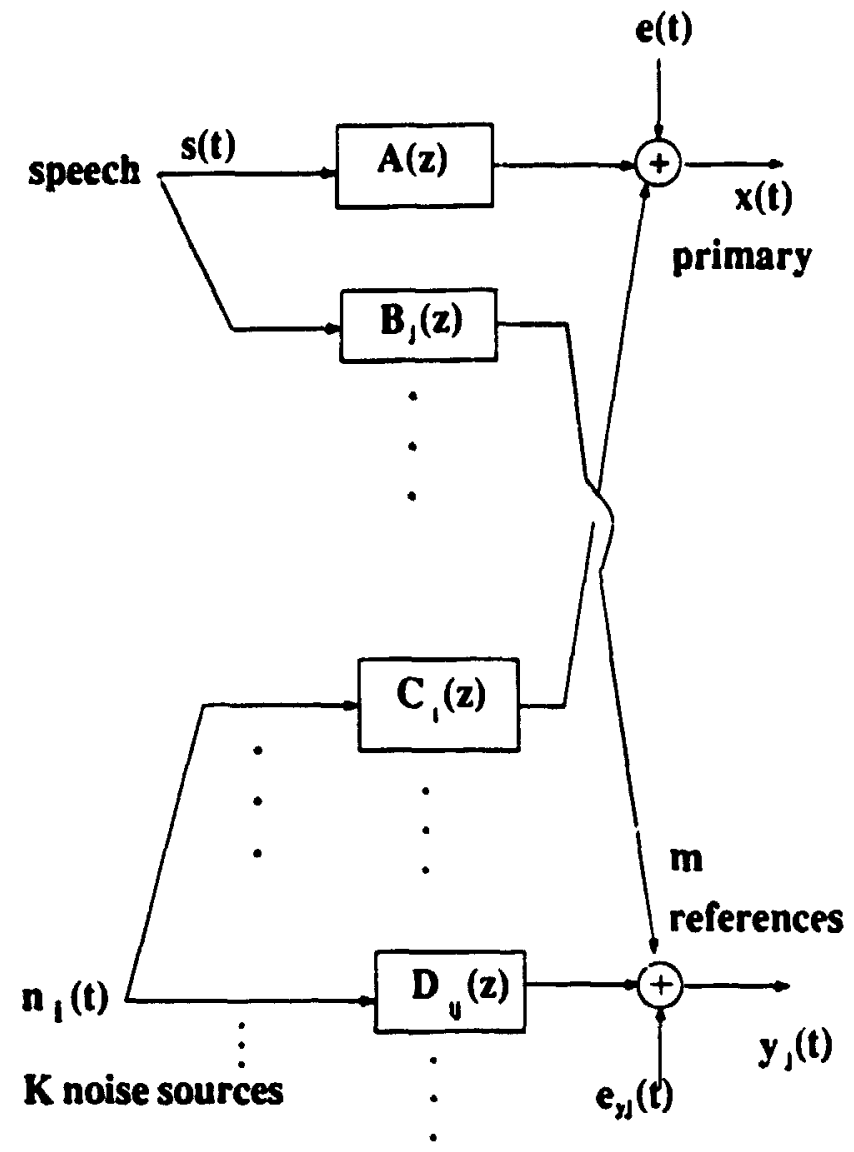

Figure 2.4 Multiple Input Multiple Output Noise System Configuration

where $\underline{s}(t)$ is a $N \times 1$ vector composed of the $N$ last speech samples

$$
s(t)=[s(t) s(t-1) \ldots s(t-N+1)]^{T}
$$

A(t) is a transfer function of length $N$. It transforms the speech prior to its capture by the primary.

$$
\underline{A}(t)=[a(t) a(t-1) \ldots a(t-N+1)]^{T}
$$


$\underline{n}(t)$ represents the $k$ noise vectors of size $(N \times 1)$ consecutively disposed in a $k N$ $x 1$ vector. Each $\mathrm{N} \times 1$ noise vector is composed of the last $\mathrm{N}$ observations of each noise source.

$$
\underline{\mathbf{n}}(\mathbf{t})=\left[\begin{array}{c}
\underline{\underline{n}}_{1}(\mathbf{t}) \\
\underline{\underline{n}}_{2}(\mathbf{t}) \\
\cdot \\
\cdot \\
\cdot \\
\underline{\underline{n}}_{k}(\mathbf{t})
\end{array}\right]
$$

where

$$
\underline{n}_{i}(t)=\left[\left.n_{i}(t) n_{i}(t-1) \ldots n_{j}(t-N+1)\right|^{T}\right.
$$

$\underline{\mathrm{C}}(\mathrm{t})$ is $\mathrm{kN} \times 1$ vector. It is composed of $\mathrm{k}$ vectors (FIR) of dimension $\mathrm{N} \times \mathrm{l}$ each. The $i^{t^{\text {th }}} N \times 1$ vector $\underline{G}_{j}(t)$, transforms the $i^{\text {th }}$ noise source vector $\underline{n}_{1}(t)$

$$
\underline{\mathbf{C}}(\mathbf{t})=\left[\begin{array}{c}
\underline{\mathbf{C}}_{1}(t) \\
\underline{\mathbf{C}}_{2}(t) \\
\cdot \\
\cdot \\
\cdot \\
\underline{\mathbf{C}}_{k}(t)
\end{array}\right]
$$

where

$$
\underline{C}_{\mathbf{i}}(t)=\left[C_{i}(t) C_{i}(t-1) \ldots C_{i}(t-N+1)\right]^{T}
$$

The observed signal at the $j^{\text {th }}$ reference is given by 


$$
y_{j}(t)=\underline{s}^{T}(t) \underline{B}_{j}(t)+\underline{n}^{T}(t) \underline{D}_{j}^{T}(t)+e_{y j}(t)
$$

where $\underline{s}(t)$ has been previously defined, $\underline{B}_{j}(t)$ is $N \times 1$ FIR vector. It transforms the speech sequence prior to its capture by the $\mathrm{j}^{\text {th }}$ reference microphone.

$$
\underline{B}_{j}(t)=\left[b_{j}(t) b_{j}(t-1) \ldots b_{j}(t-N+1)\right]^{T}
$$

$\mathbf{D}_{\mathbf{J}}(t)$ is a $\mathbf{k N} \times 1$ vector composed of $k$ FIRs of length $\mathrm{N}$ sequentially disposed. This vector transforms the $\mathbf{k}$ noise source sequences disposed in $\underline{n}(\mathbf{t})$ prior to their capture by the $\mathrm{j}^{\text {th }}$ reference microphone.

$$
\underline{D}_{j}(t)=\left[\begin{array}{c}
\underline{d}_{1 j}(t) \\
\underline{d}_{2 j}(t) \\
\cdot \\
\cdot \\
\cdot \\
\underline{d}_{k j}(t)
\end{array}\right]
$$

where $\underline{d}_{v}(t)$ is a $N \times 1$ FIR vector. It transforms the $i^{\text {th }}$ noise source sequence $\underline{n}_{i}(t)$ prior to its capture by the $\mathrm{j}^{\text {th }}$ reference microphone.

$$
\underline{d}_{i j}(t)=\left[d_{i j}(t) d_{i j}(t-1) \ldots d_{i j}(t-N+1)\right]^{T}
$$

The ANC uses a sequence of $\mathrm{N}$ observations from each reference microphone. It then compares it with the primary signal. Therefore, a $N \times 1$ vector representing a sequence of $\mathrm{N}$ observations is defined for each reference. Hence for the $\mathrm{j}^{\text {th }}$ reference microphone

$$
\underline{y}_{j}(t)=\left[y_{j}(t) y_{j}(t-1) \ldots y_{j}(t-N+1)\right]^{T}
$$


Further, it is more convenient to define an observation matrix of dimension $\mathrm{N} \times \mathrm{m}$ which is represented by

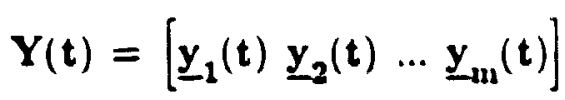

In order to clearly jefine the system's limitations and to ease analytical derivations, only one reference microphone is considered. Also, it is assumed that the ANC adapts during speechless period only. This was suggested in [5]. Therefore, the system's equations are simplified. At the primary, the observed noise process is obtained using equation $(2.1)$ by setting $\underline{s}(t)=0$.

$$
x(t)=\underline{n}^{T}(t) \underline{C}^{T}(t)+e_{x}(t)
$$

at the reference, the $\mathrm{N}$ sample sequence of the noise process is obtained using (2.8) in (2.12)

$$
\underline{\underline{y}}(t)=\left[\begin{array}{c}
\underline{n}^{T}(t) \underline{D} \\
\underline{n}^{T}(t-1) \underline{D} \\
\cdot \\
\cdot \\
\cdot \\
\underline{n}^{T}(t-N+1) \underline{D}
\end{array}\right]+\underline{e}_{y}(t)
$$

where 


$$
\underline{N}(t)=\left[\begin{array}{c}
\underline{n}^{T}(t) \underline{D} \\
\underline{n}^{T}(\underline{\underline{D}}) \underline{\underline{D}} \\
\cdot \\
\cdot \\
\cdot \\
\underline{n}^{T}(t-N+1) \underline{D}
\end{array}\right]
$$

and $e_{y}(t)$ is a $N \times 1$ sequence of the $N$ last approximation errors of $y(t)$. This error is assumed to be an independent random process with zero mean and variance $\sigma_{\mathrm{ey}}{ }^{2}$.

The linear regression concept states that there exists a linear combination of the $N$ last observations at iul reference microphone such that it can reproduce $x(t)$, the observed noise process at the primary. For doing so, a $\mathrm{Nxl}$ vector $\underline{W}(\mathrm{t})$ is defined. Hence the desired signal estimation (the primary signal) is

$$
\overline{\mathbf{x}}(\mathbf{t})=\underline{\mathbf{y}}^{\mathbf{T}}(\mathbf{t}) \underline{\mathbf{W}}(\mathbf{t})
$$

the estimation error is the difference between the actual signal $\mathbf{x}(\mathbf{t})$ and its estimate $\tilde{\mathbf{x}}(\mathbf{t})$

$$
e(t)=x(t)-\bar{x}(t)
$$

The aim of linear regression is to minimize the estimation error in a mean square sense. This is done with respect to the transfer function coefficients $\underline{W}(t)$. Hence the mean square error (mse) is obtained by squaring (2.19) and by taking the expectation on both side of the resulting equation.

$$
\text { mse }=E\left[e^{2}(t)\right]=E\left[x^{2}(t)-2 x(t) \tilde{x}(t)+\tilde{x}^{2}(t)\right]
$$




$$
=E\left[x^{2}(t)\right]-2 E[x(t) \bar{x}(t)]+E\left[\tilde{x}^{2}(t)\right]
$$

For ease of analysis, it is assumed that every transfer function are constant over the period of observation. Hence the transfer functions are irdependent of time and are not affected by the expectation operator. Substituting (2.14), (2.16) and. (2.18) in (2.21), the mse is now expressed in term of the noise processes.

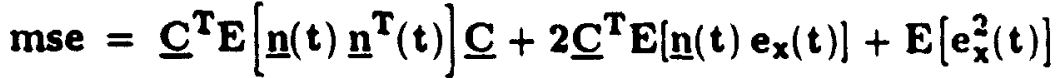

$$
\begin{aligned}
& -2 \underline{C}^{T} E\left[\underline{n}(t) \underline{N}^{T}(t)\right] \underline{w}-2 E\left[e_{x}(t) \underline{N}^{T}(t)\right] \underline{w} \\
& -2 E\left[e_{x}(t) \underline{e}_{y}(t)\right] \underline{w}+\underline{W}^{T}(t) E\left[\underline{N}(t) \underline{N}^{T}(t)\right] \underline{w} \\
& +2 \underline{W}^{T} E\left[\underline{e}_{y}(t) \underline{N}^{T}(t)\right] \underline{w}+\underline{w}^{T} E\left[\underline{e}_{y}(t) \underline{e_{y}^{T}}(t)\right] \underline{w}
\end{aligned}
$$

As stated earlier, $e_{x}(t)$ and $e_{y}(t)$ are independent random processes with zero mean and respective variance $\sigma_{\mathrm{ex}}{ }^{2}$ and $\sigma_{\mathrm{ey}}{ }^{2}$. Hence the mse becomes

$$
\begin{aligned}
& \text { mse }=\underline{C}^{T} E\left[\underline{n}(t) \underline{n}^{T}(t)\right] \underline{C}+\sigma_{e x}^{2}-2 \underline{C}^{T} E\left[\underline{n}(t) \underline{N}^{T}(t)\right] \underline{W} \\
& +\underline{w}^{T}(t) E\left[\underline{N}(t) \underline{N}^{T}(t)\right] \underline{w}+\underline{w}^{T} E\left[\underline{e}_{y}(t) \underline{e}_{y}^{T}(t)\right] \underline{w}
\end{aligned}
$$

where $E\left[\underline{n}(t) \underline{n}^{T}(t)\right]$ is the autocorrelation of the noise processes vector. Assuming that each noise process has zero mean and that they are independent from one another, the autocorrelation of the noise becomes

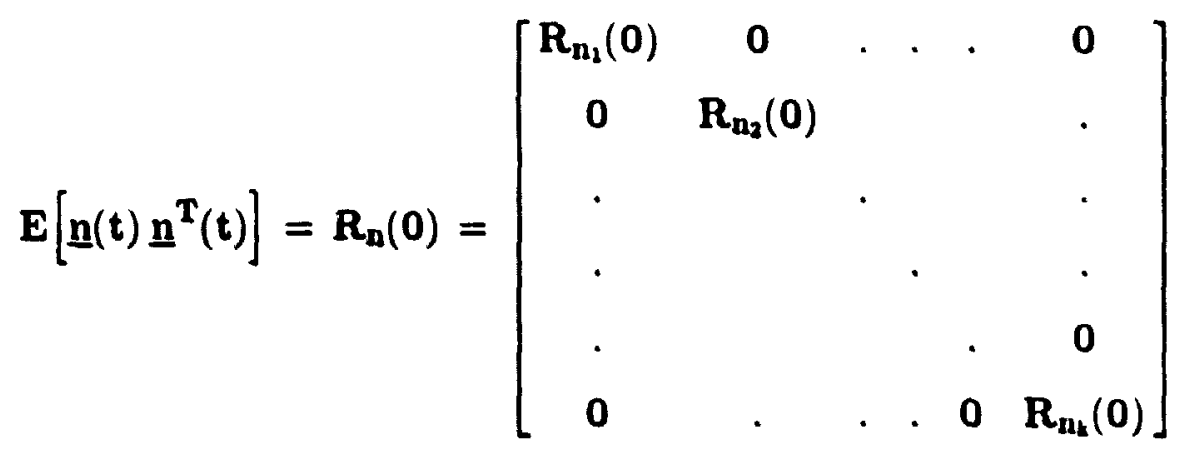


where

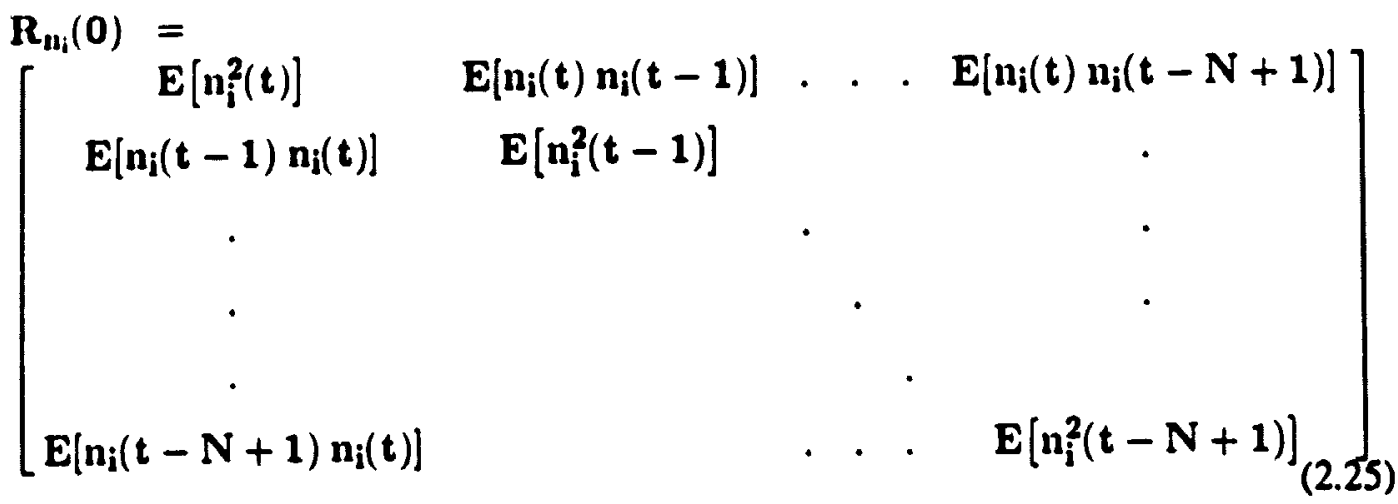

$\mathbf{E}\left[\mathbf{N}(t) \underline{N}^{T}(t)\right]$ is the autocorrelation of the noise process observed at the reference.

$$
\begin{aligned}
& R=E\left[\underline{\mathbf{N}}(t) \underline{\mathbf{N}}^{\mathbf{T}}(t)\right]=
\end{aligned}
$$

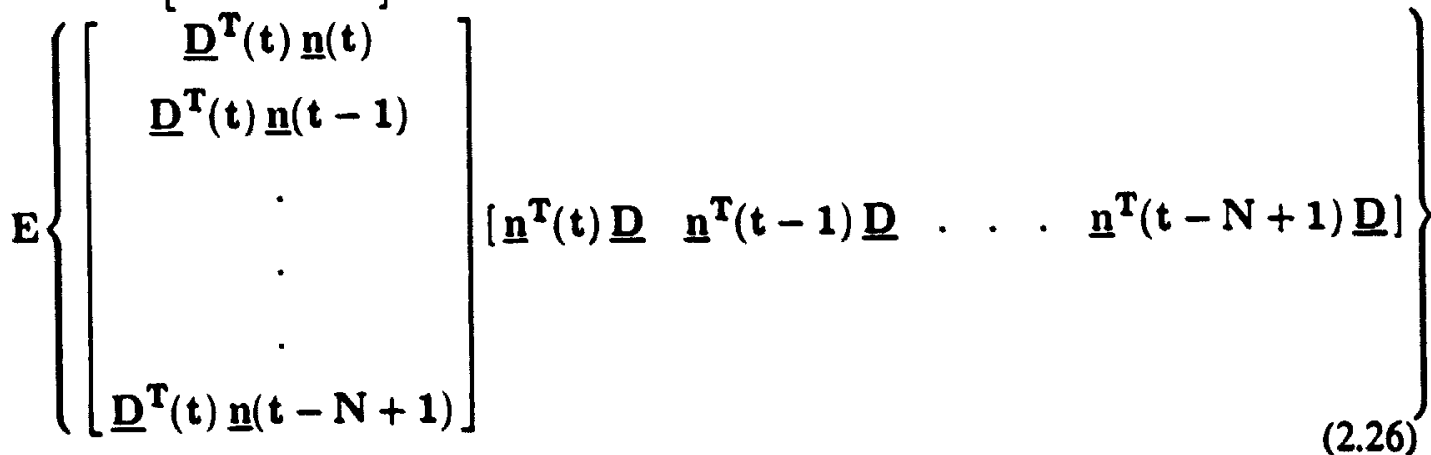

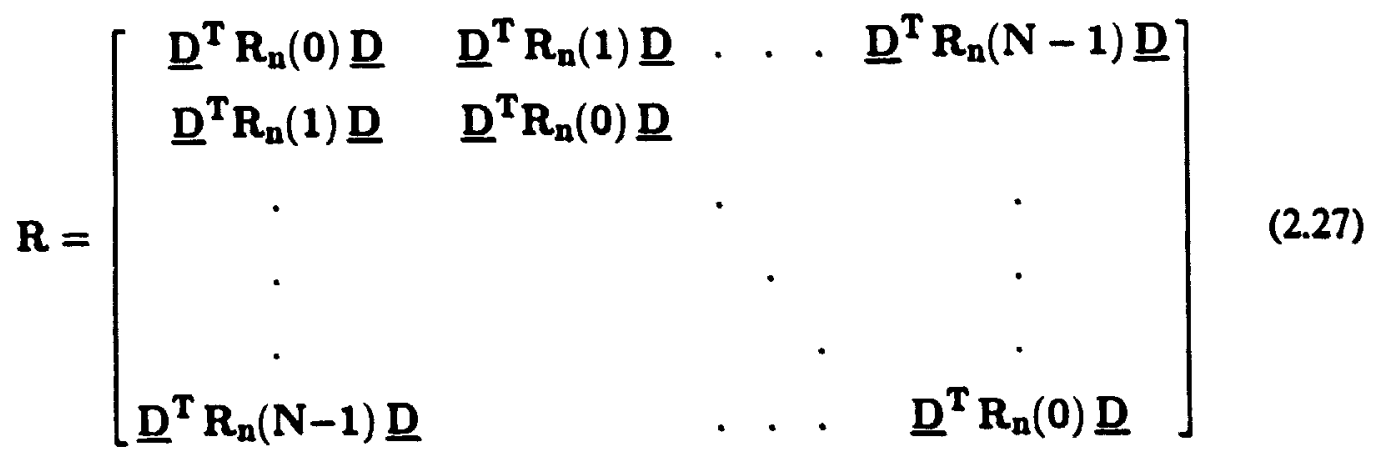

where 


$$
R_{n}(\mathbf{i})=E\left[\underline{n}(t) \underline{n}^{T}(t-i)\right]
$$

$\underline{C}^{T} \mathbf{E}\left[\underline{n}(t) \underline{N}^{T}(t)\right]$ is the cross-correlation between the primary and the reference microphones with respect to the noise sources. A vector $\mathbf{p}$ representing the crosscorrelation is defined

$$
\underline{p}=\underline{C}^{T} \mathbf{E}\left[\underline{n}(t) \underline{N}^{T}(t)\right]
$$

$$
\underline{p}=\underline{C}^{T} \mathbf{E}\left\{\underline{n}(t)\left[\left.\underline{n}^{T}(t) \underline{D} \underline{n}^{T}(t-1) \underline{D} \cdot \cdot \cdot \underline{n}^{T}(t-N+1) \underline{D}\right|^{T}\right\}\right.
$$

Substituting equations (2.28) in (2.30), the cross-correlation $p$ becomes

$$
\underline{p}=\left[\begin{array}{c}
\underline{C}^{T} R_{n}(0) \underline{D} \\
\underline{C}^{T} R_{n}(1) \underline{D} \\
\cdot \\
\cdot \\
\cdot \\
\underline{C}^{T} R_{n}(N-1) \underline{D}
\end{array}\right]
$$

$E\left[\underline{e_{y}}(t) \underline{e_{y}} \mathbf{T}(t)\right]$ is the autocorrelation matrix of the approximation sequence error at the reference microphone. Using $e_{y}(t)$ 's properties, the autocorrelation becomes

$$
\mathbf{R}_{\text {ey }}=E\left[\underline{e}_{y}(t) \underline{e_{y}} T_{(t)}\right]=\sigma_{c y}^{2} I
$$

where $I$ is an $\mathbf{N x N}$ identity matrix.

The mse may now be expressed in a more compressed form. Substituting equations (2.24), (2.26), (2.29) and, (2.32) in (2.23), the mse is 
mse $=\underline{\mathbf{C}}^{\mathrm{T}} \mathbf{R}_{\mathrm{n}}(0) \underline{\mathbf{C}}+\sigma_{\mathrm{ex}}^{2}-2 \mathrm{p} \underline{\mathrm{W}}+\underline{\mathrm{W}}^{\mathrm{T}}+\underline{\mathbf{W}}^{\mathrm{T}} \mathrm{R}_{\mathrm{cy}} \underline{\mathrm{W}} \quad \underline{\mathrm{W}} \mathbf{R} \underline{\mathrm{W}}(2.33)$

Recall that the mse is strictly positive. As indicated in [13], the filter coefficients $\underline{W}(t)$ has the shape of a hyperbolic function facing upward. The limits of that function is the mean square error obtained for a particular $\underline{W}(t)$. The bottom of the function coincides with the minimum mean square error (mmse). Hence, this point is obtained by using the optimum filter coefficients $\underline{W}_{\text {opt. }}$. The mmse is obtained by taking the partial derivative of (2.33) with respect to $\underline{W}(t)$ and setting it to zero.

$$
\frac{\delta m s e}{\delta \underline{W}}=-2 \underline{p}+2 R \underline{W}_{o p t}+2 R_{e y} \underline{W}_{o p t}=0
$$

hence

$$
\left(\mathbf{R}+\mathbf{R}_{\text {oy }}\right) \underline{\mathbf{W}}_{\text {opt }}=\underline{\mathbf{p}}
$$

(2.35) is the well known Wiener-Hopf equation [14]. By inspection, $\mathbf{R}_{\mathrm{ey}}$ introduces a bias in the estimation. Obviously, the optimum solution should not take into account the effect of $\boldsymbol{R}_{\mathbf{e y}}$. However this reinforce the fact that the approximation error $\mathbf{e}_{\mathbf{y}}(\boldsymbol{t})$ constitute a limiting factor. Therefore any adaptive noise canceller (ANC) using linear regression, is limited by this factor. Assuming that the approximation error at the reference is small enough so that

$$
\mathbf{R} \gg \mathbf{R}_{\text {ey }}
$$

then, (2.35) may be approximated to

$$
\mathbf{R} \underline{\mathbf{W}}_{\text {opt }} \approx \underline{\mathbf{p}}
$$


Substituting (2.27) and (2.31) in (2.36)

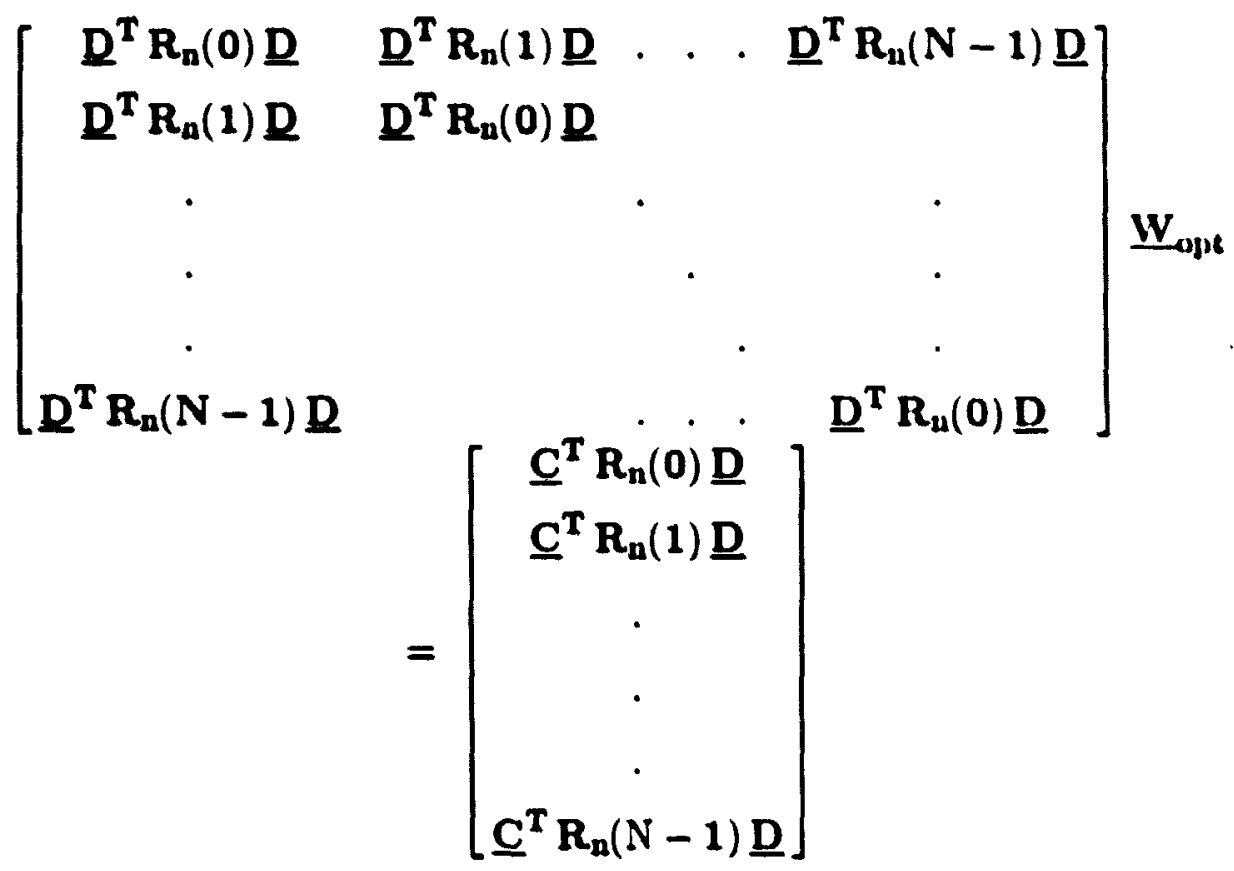

Equation (2.37) illustrates how the noise process observed at the reference micro phone can be used to estimate the noise process at the primary. It is evident that $\underline{W}_{0,4}$ bridges the gap which exists between the two transfer functions $\underline{\mathbf{C}}$ and $\underline{\mathbf{D}}$. The who:e process is done using a sequence of $\mathrm{N}$ samples. This is the exact same length of $t^{\prime \prime} \mathrm{r}$ FIRs defined earlier.

\subsection{Model Constraints for Successful Adaptive Noise Sancellation}

The mathematical model described in the previous section provides a qualitalive. description of the background acoustic noise system. The concept of noise cancellat.1.3. is based on the correlation thi' exists between the captured noise at the primary ni those captured at the references $[2-6,8,11,13,14,18,20,21,25,26,31,33,34,38,45,46$.$) .$ $53,56,57,69]$. First the transfer functions linking the references to the primary 1 sis 
estimated. Then using these transfer functions, the estirnated noise is subtracted from the primary. Figure 1.3. illustrates the concept of adaptation.

The adaptive noise cancellation system will not work if some minimum model constraints are not met. $F$ t the observed noise at the primary must be reconstructible by a linear combination of the observed noise at the references. This is the basis of linear regression. Second, the concept of causality must be met. For an observer sitting at the reference, the noise process must occur before the one at the primary. However, the system could be non-causal to some extent. As mentioned in [1], this can be overcome by adding a delay at the primary microphone. Also, if the process is periodic over a long time frame, this will compensate for non-causality. In that case, only the phase lag matier. As a minimum, if these two constraints are not met then, the adaptive noise cancellation is not feasioie. It will be shown later how this problem can be alleviated by collocating the reference microphones with respect to the noise sources. 


\section{Chapter 3 Recording set up and Testing}

\subsection{Introduction}

This chapter covers the recording setups and the testing conditions used during the data acquisition phase. The data analysis which is carried out in a later chapter depends entirely on this critical phase.

This chapter is divided into 4 parts. The first part describes the position of each microphone with respect to its system's contribution. The second part covers the entire equipment setup used throughout the data acquisition phase. In the third part, tests which validate the equipment accurately are briefly presented and discussed. The last part talks about the various driving conditions.

\subsection{Microphone Locations}

The selection of each microphone location is probably the most critical operation of the entire research. If badly selected, the noise analysis and hence any course of action stemming from it could lead to inconclusive results. In order to minimize the risk of error, some basic criteria regarding the microphones placement are defined. Chapter 2 established a noise model composed of many noise sources. The model is pertinent to the normal use of a car. Logically, the aim is to capture as many different noise processes as possible using the reference microphones. This would ensure that every noise process 
perceived at the primary is at least observed at the references. Also, it is desirable to get an acoustic barrier between the primary and any of the reference microphones.

Another criterion is the placing of reference microphones as close as possible to the primary. Being in a multi-noise system, the complexity of the differential transfer function linking two points grows with the distance betweer them. Hence, minimizing this distance contributes to reducing the transfer function complexity between the primary and the reference $[1,2,20,21,25,26,69]$.

The two criteria mentioned above are contradictory. On one hand, it is desired to acoustically isolate the various microphones. The isolation can be achieved by increasing the distance between the microphones. On the other hand, it is desired to place the microphones as close as possible to the primary. Therefore, the selection of the microphone locations is a trade-off between these two requirements.

Throughout every tests, omni-directional microphones are used. Figure 3.1 shows the microphone configuration is as follows.

Microphone \# 1 (reference \#1) is located in the engine compartment. It is placed right next to the fire wall and the hood. Also microphone \# 1 is collocated with the fan motor. Its main purpose is to pick up the engine noise while being free of the other processes especially speech. Its distance with respect to the primary is about $20 \mathrm{~cm}$ with both microphones facing in opposite directions.

Microphone \# 2 (reference \# 2) is located under the steering wheel in a small glove compartment. Being in an intermediate location between the engine compartment and the primary, it should have less noise than microphone \#1 which is located inside the engine compartment. By listening tests, it was found that when the background acoustic noise is low, speech can be coupled to microphone \# 2, which is facing in an opposite direction with respect to the primary. A distance of about $20 \mathrm{~cm}$ separates it from the 


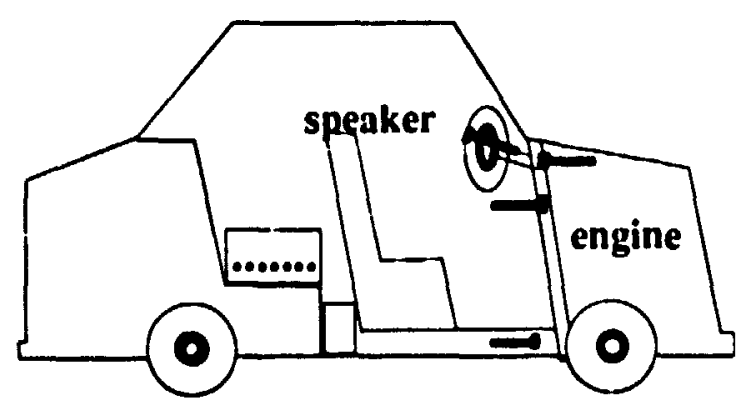

Figure 3.1 Microphone Disposition During Field Tests

primary.

Microphone \# 3 is placed under the driver floor mat. It should pick up any road noise. By being inside the cabin, its differential transfer function with respect to the primary is minimized. There is about 1 meter separating microphones \# 3 and the primary.

Microphone \# 4 is chosen for being the primary. The microphone is located at the steering wheel facing the driver. A distance of about $20 \mathrm{~cm}$ separates the speaker from the microphone.

\subsection{Equipment Set Up}

The same equipment is used in all tests. Figure 3.2 illustrates the equipment setup. The microphones output are fed through a pre-amplifier to an 8 track tapt: recorder. The pre-amplifier has a flat gain over the acoustic band. Ron Belaire built the device as part of his fourth year project [70]. The RACAL 8 track tape recorder allows simultaneous recording from the four microphones. Prior to sampling the acquired data, a synchronization pulse is inserted at the beginning of each test sequence. By doing so, each channel can be sequentially sampled. A Low-Pass Butterworth filter with a cutoff frequency set at $3.7 \mathrm{KHz}$ is used to remove aliasing prior to sampling. The sampling 


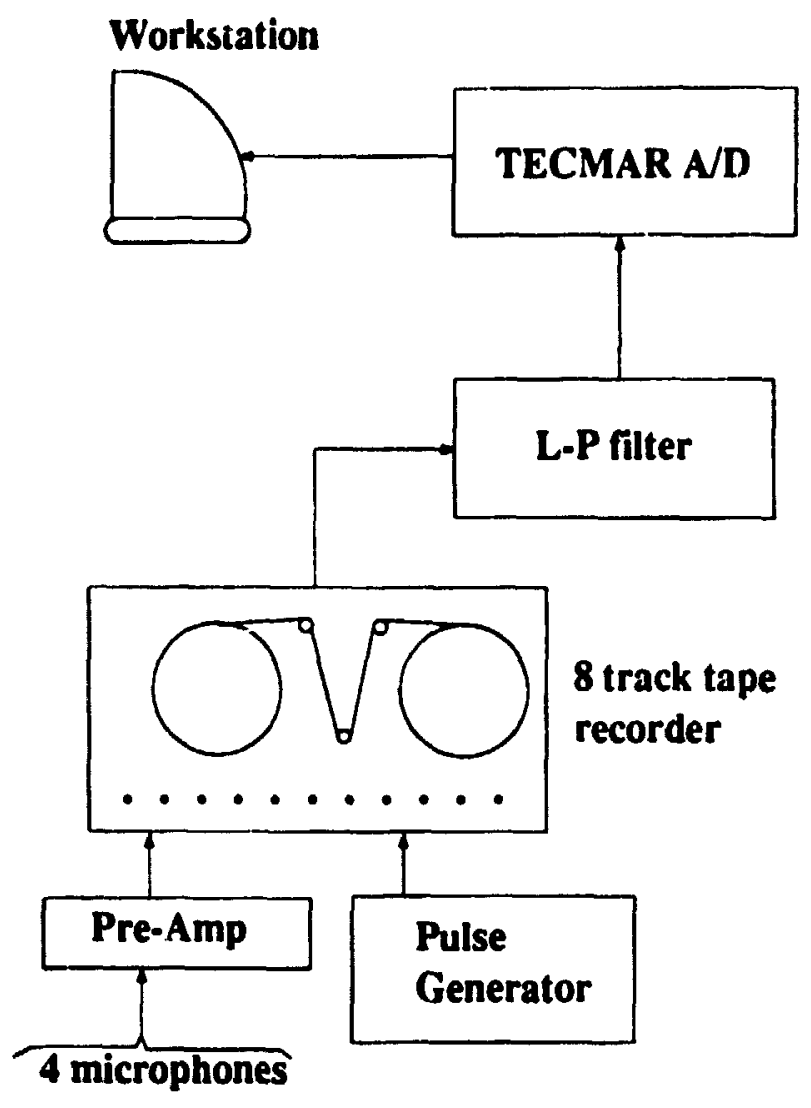

Figure 3.2 Recording Set-Up Configuration

is performed at a rate of $10 \mathrm{KHz}$ using the TECMAR AVD board. Windows of $52 \mathrm{~K}$ samples are saved in a file then transferred to a SUN and PC workstations where it is analyzed using the MATLAB package.

\subsection{Measurement Set Up Validation}

The equipment was validated through laboratory testing. Each component was individually tested. The basic tests consisted in generating various sine waves at 
frequencies in the acoustic band. The MATLAB package served as a tool for validating and analyzing the data.

First the TECMAR board was tested. Then, the tape recorder was inspected. It was found that the tape recorder inputs could easily saturate due to the absence of an automatic gain controller. Therefore, close monitoring was required during recording. This could lead to a potei, ial problem. The equipment background electronic noise is too small with respect to the observed signals. However gaging down the input power signal of a microphone in order to avoid saturation could results in burying a low power noise signal into the background electronic noise. If this happens, adaptation could be difficult to ashieve using this noise signal.

The next step consisted in adding the microphones and the pre-amrlifier unit. This step was repeated for each of the tape recorder track. Every microphone used was omnidirectional. The pre-amplifier unit was built by Ron Belaire as part of a project in noise cancellation. The device had a flat gain up to $20 \mathrm{KHz}$ which was more than enough.

Some microphone tests were performed with and without the tape recorder. The final step consisted in reproducing the field set-up. Figure 3.2 shows the configuration used. Two components were added. A 12 Volt battery and a DC/AC conventer were used as power supply. Still, the equipment was compliant.

After a preliminary analysis of the data, a test was performed on the recorded data using a spectrum zalyzer. The test confirmed the data validity.

\subsection{Driving Conditions}

We selected the driving conditions such that a few noise processes could be observed individually. Then we varied the conditions so that the processes could be added 
gradually. It wasn't always possible to isolate specific noise processes. Note that only the basic noise processes surrounding the normal car use were considered. Each tests were performed twice: with the fan off and then with the fan on. The driving conditions are indicated in table 3.1 .

\begin{tabular}{|l|l|}
\hline engine off, & fan on \\
\hline engine idling, car static & fan off \\
\hline engine idling, car static & fan on \\
\hline car speed $60 \mathrm{Km} / \mathrm{hr}$ on a blvd & fan off \\
\hline car speed $60 \mathrm{Km} / \mathrm{hr}$ on a blvd & fan on \\
\hline car speed $100 \mathrm{Km} / \mathrm{hr}$ on a freeway & fan off \\
\hline car speed $100 \mathrm{Km} / \mathrm{hr}$ on a freeway & fan on \\
\hline
\end{tabular}

Table 3.1 Driving Conditions During Field Tests

These tests allow the analysis of the engine noise alone and the fan noise alone. These two noise sources are the only one which can be analyzed independently of the others. For example, the road noise is always accompanied by the engine noise. The recording level set during the field tests are indicated in table 3.2. 


\begin{tabular}{|l|l|l|l|}
\hline test & location & fan off & fun on \\
\hline car idling & under the hood & .5 Volt & 1 Volt \\
\hline car idling & under the steering & .2 Volt & .2 Volt \\
\hline car idling & under floor mat & .1 Volt & .1 Volt \\
\hline car idling & at the steering & .2 Volt & .2 Volt \\
\hline $60 \mathrm{Km} / \mathrm{hr}$ & under the hood & .5 Volt & .5 Volt \\
\hline $60 \mathrm{Km} / \mathrm{hr}$ & under the steering & .2 Volt & .2 Volt \\
\hline $60 \mathrm{Km} / \mathrm{hr}$ & under floor mat & .2 Volt & .2 Volt \\
\hline $60 \mathrm{Km} / \mathrm{hr}$ & at the steering & .2 Volt & .2 Volt \\
\hline $100 \mathrm{Km} / \mathrm{hr}$ & under the hood & 1 Volt & 1 Volt \\
\hline $100 \mathrm{Km} / \mathrm{hr}$ & under the steering & .2 Volt & .2 Volt \\
\hline $100 \mathrm{Km} / \mathrm{hr}$ & under floor mat & .2 Volt & .2 Volt \\
\hline $100 \mathrm{Km} / \mathrm{hr}$ & at the steering & .2 Volt & .2 Volt \\
\hline engine off & under the hood & - & 1 Volt \\
\hline engine off & under the steering & - & .1 Volt \\
\hline engine off & under floor mat & - & .1 Volt \\
\hline engine off & at the steering & - & .1 Volt \\
\hline
\end{tabular}

Table 3.2 Recording Level During Field Tests 


\section{Chapter 4 Data Analysis}

\subsection{Introduction}

This chapter analyzes the background acoustic noise recorded in a medium size ca. It focuses only on normal driving conditions. Due to the complexity of the background acoustic noise, the analysis is performed in a qualitative manner. The results obtained in both the time and frequency domain will be used as guidelines for designing various ANC algorithms. A second aim is to evaluate in a qualitative manner the possibility of using a multiple reference microphone ANC.

First, the analysis considers the noise power spectrum density (PSD). Then, the acoustic band is split into an upper and lower band (above and below $1.5 \mathrm{KHz}$ ). Correlation tests are performed on both bands.

\subsection{Power Spectral Density}

The test conditions under which the data was acquired are described in table 3.1 . The PSD is obtained by applying the Welsh method [12] on a window of 4096 samples. Hence the PSD is averaged over a period of about 410 msec. The resulting curve is presented using 64 frequency bins linearly spaced from 0 to $5000 \mathrm{~Hz}$. The frequency domain shows that under various driving conditions, each microphone has a particular spectral signature. 
In chapter 2 a noise model was developped. It shows that each noise process goes through a transfer function prior to reaching an observation point (i.e. microphone location). For, Goubran et al. [1,2] indicated that the car cabin acts as a low pass filter. The low-pass filter cut-off frequency varies from car to car. Figure 4.1 shows the PSD of the engine noise observed from four locations. Microphone \# 1 (located under the hood) is collocated with the noise source. The other microphone placed inside the cabin have a low-pass filtered PSD with respect to microphone \#1. The cabin cut-off frequency with respect to the primary (at the steering wheel) is about $450 \mathrm{~Hz}$. Other spectral characteristics are observed, the most important ones are stated below.

As shown in figures 4.1 through 4.4, the reference microphone locations have some spectral signature properties. Only the observable peaks (common to the primary microphone PSD) are considered i.e. there may be some peaks buried in with uncorrelated noise but they are not considered in this chapter.

The PSD of microphone \# 1 (under the hood) is composed of peaks distributed over the entire acoustic band. Two peaks are common to the primary microphone (at the steering wheel). The first peak is located below $1.5 \mathrm{KHz}$. The second peak is located at about $3 \mathrm{KHz}$ when the car moves at $60 \mathrm{Km} / \mathrm{hr}$. This peaks moves to near $3.5 \mathrm{KHz}$ as the car's speed is increased to $100 \mathrm{Km} / \mathrm{hr}$. This is believed to be the air flow effect on and around the car. A simple listening test confirms this assumption.

Microphone * 2's (under the steering wheel) PSD is mainly composed of a low frequency peak located below $1.2 \mathrm{KHz}$.

Microphone \# 3's (under the driver floor mat) PSD is composed of two peaks. The main peak is below $1 \mathrm{KHz}$. The second peak appears only when the car moves. It is located at $1700 \mathrm{~Hz}$. As the car's speed increases, the peak remains at the sume frequency but its amplitude increases. 
Mic. \# 1 Under the Hood

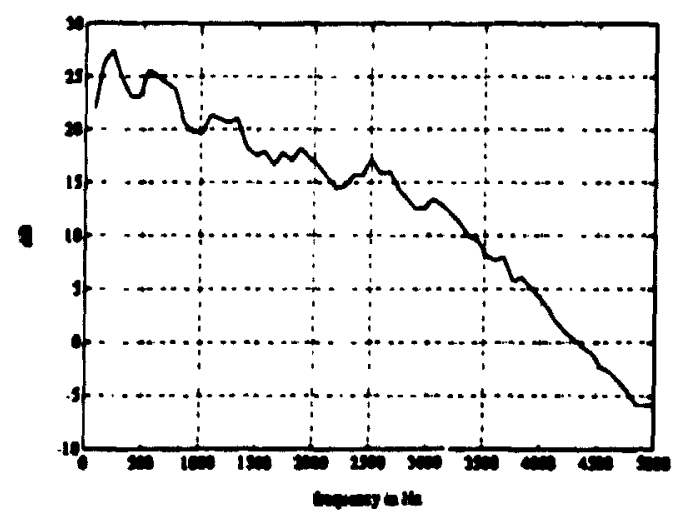

a.

Mic. \# 3 Undor Floor Mat

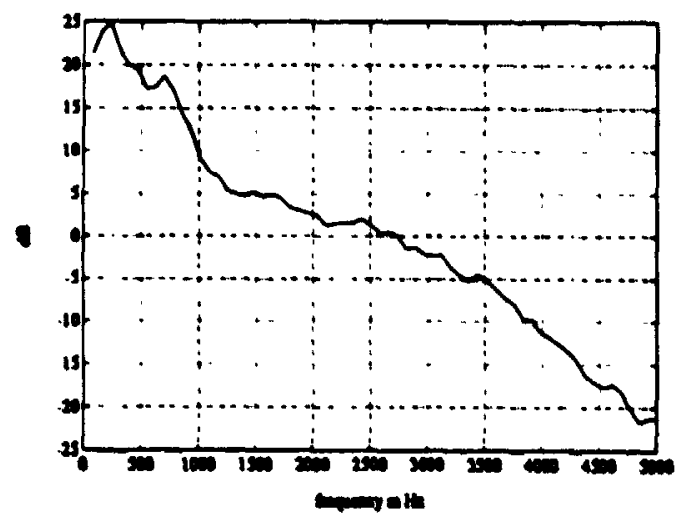

c.
Mic. \# 2 Under the Steering

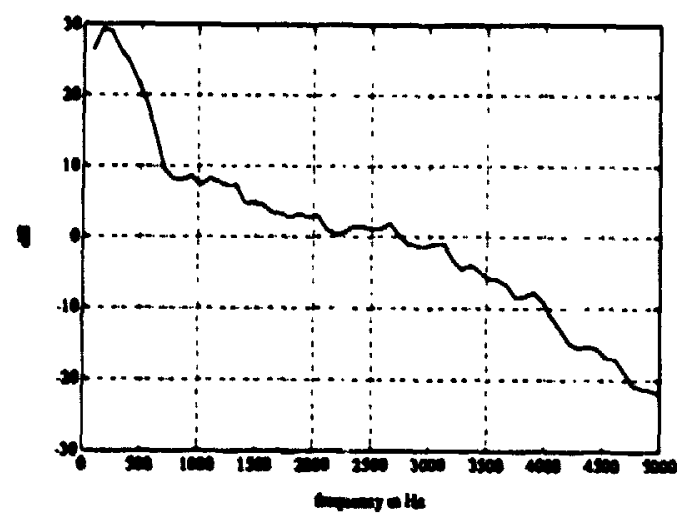

b.

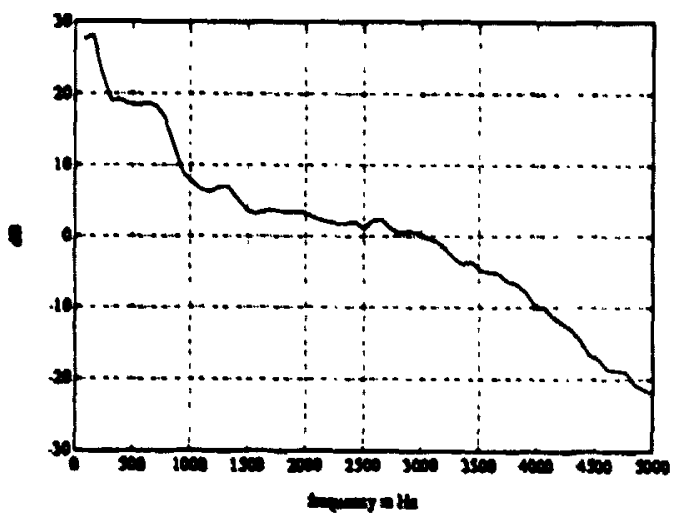

d.

Figure 4.1 Power Spectrum Density, Car Idling, Fan Off

From the various spectral signature observations, it seems that the upper and lower bands have different noise processes. Hence a particular reference microphone could offer its spectral signature for noise cancellation. The important point is to determine if 
Mic. \# 1 Under the Hood

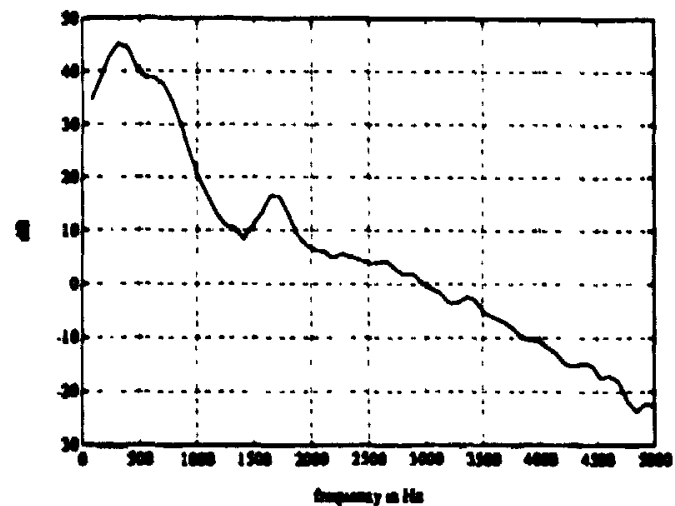

a.

Mic. \# 3 Under Floor Mat

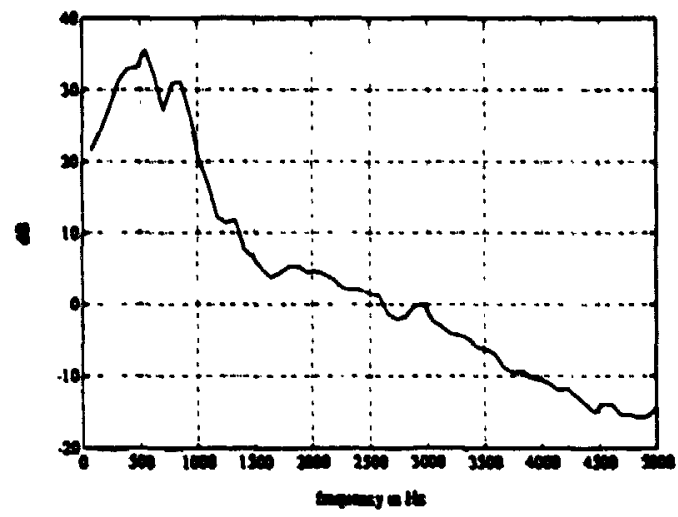

c.
Mic. \# 2 Under the Steenng

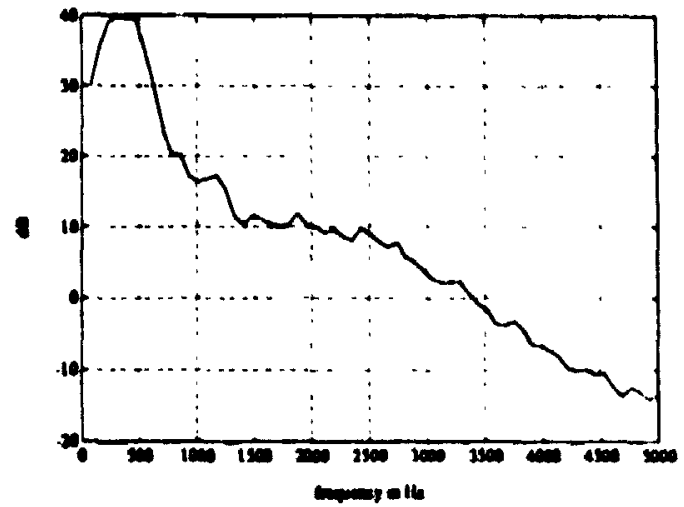

b.

Mic. \# 4 At Steering (Primary)

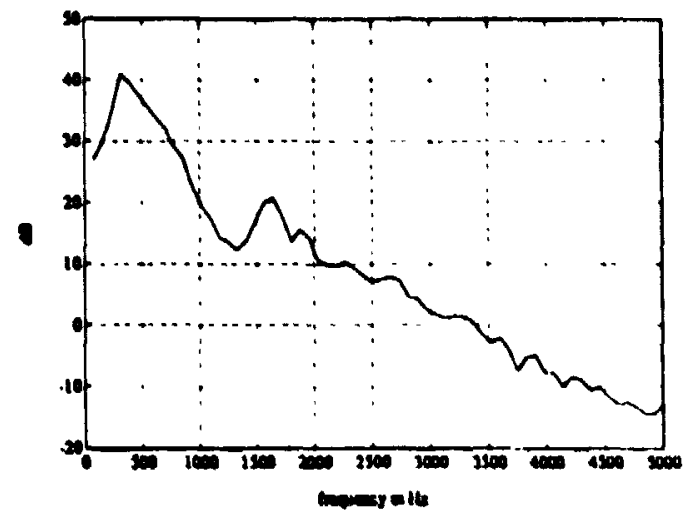

d.

Figure 4.2 Power Spectrum Density, Engine Off, Fan On

a degree of correlation exists between a spectral signature observed at a reference and at the primary microphone. 
Mic. * 1 Under the Hood

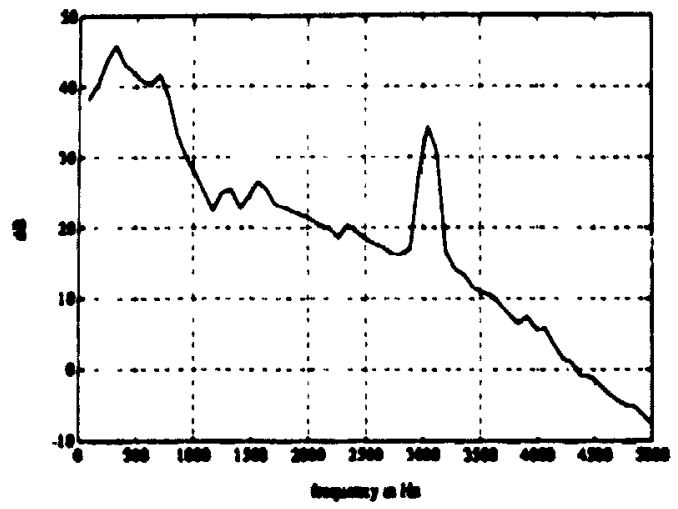

a.

Mic. * 3 Under Floor Mat

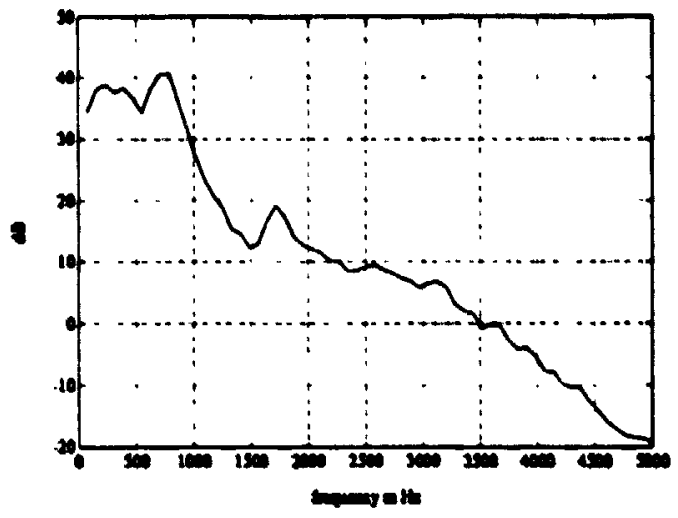

c.
Mic. \# 2 Under the Steering

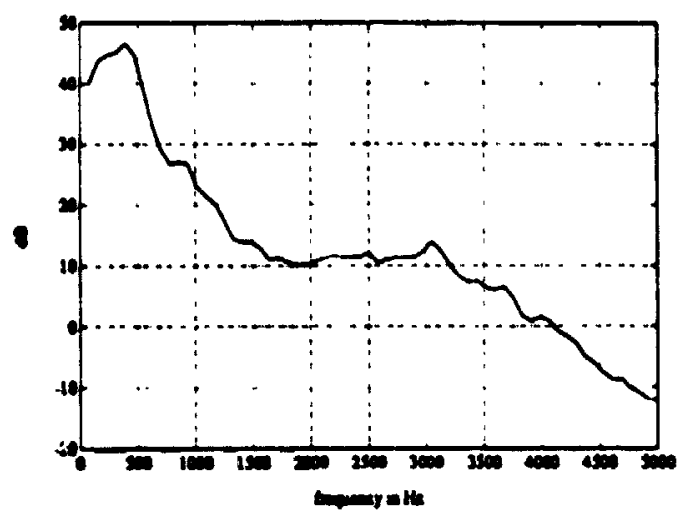

b.
Mic. 4 At Steering (Primary)

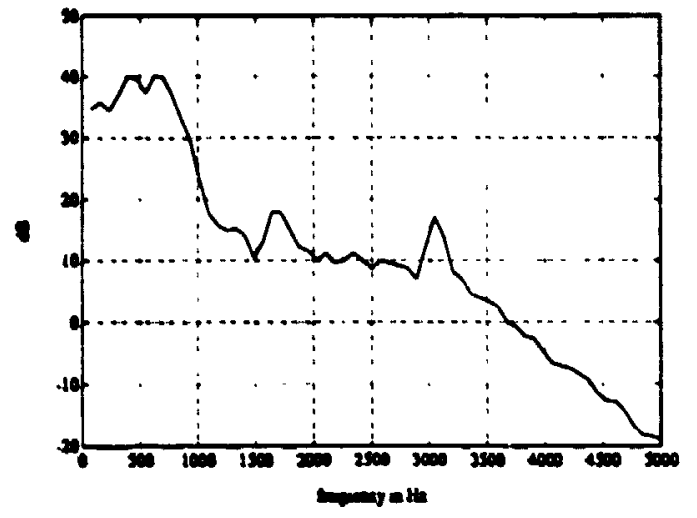

d.

Figure 4.3 Power Spectrum Density, Car Speed $60 \mathrm{Km} / \mathrm{hr}$, Fan On

\subsection{Correlation}

As mentioned in an earlier chapter, the ANC effectiveness depends on the degree of correlation that exists between the reference and the primary microphones. Due to 
Mic. \# 1 Under the Hood

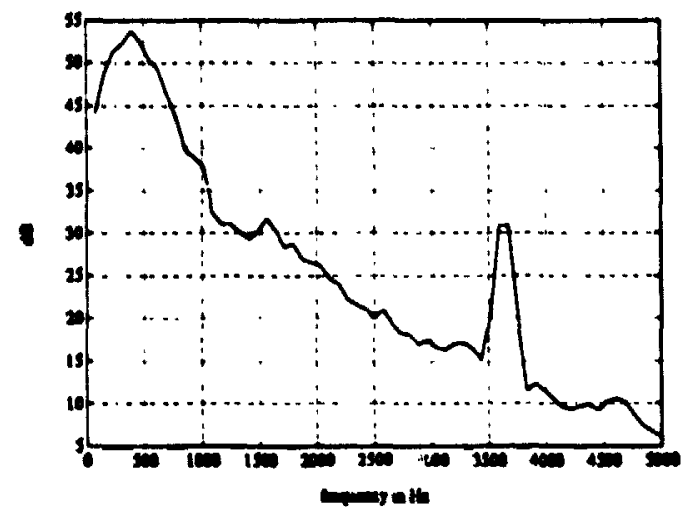

a.

Mic. \# 3 Under Floor Mat

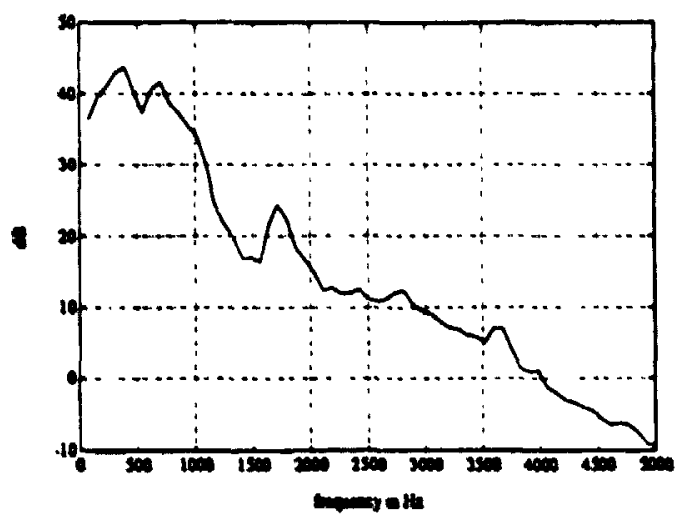

c.
Mic. \# 2 Under the Steering

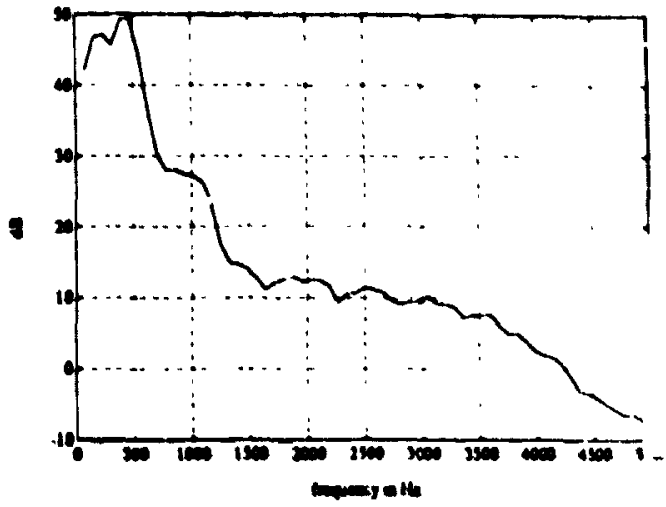

b.
Mic. \# 4 At Steering (Primary)

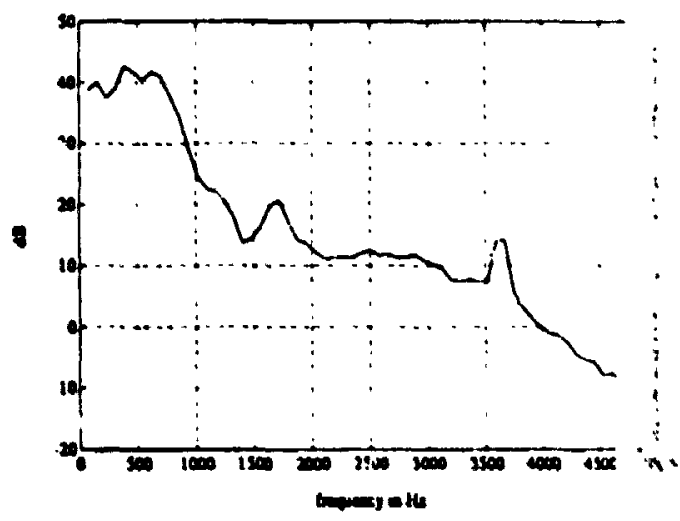

d.

Figure 4.4 Power Spectrum Density, Car Speed $100 \mathrm{Km} / \mathrm{hr}$, Fan On

the complexity of background acoustic noise characteristics, the time domain a $.2 \cdot 4.45$ can only provide some guidelines for the conception of an ANC. There are two a $\cdots, \cdots$ concern: the signal periodicity and the degree of correlation existing between a pa ...uliar 
reference microphone and the primary microphone.

\subsubsection{Autocorrelation}

The autocorrelation is evaluated using a 2048 point FFT method. A sliding window of 2048 points was applied over 100 time steps. For each step, the correlation (and cross-correlation) is evaluated. The resulting curve is the average of the 100 time step curves.

Figures 4.5 and 4.6 show that the noise processes observed in the lower and upper bands, have a certain degree of neriodicity. This indicates that the differential delays betwee: a reference microphone and the primary microphone become, to some extent, a function of the phase. Hence, the compensating delay which must be inserted for ensuring coherence (or system causalit;') between the primary and the reference signal could be shortened.

\subsubsection{Cross-Correlation}

The cross-correlation indicates the degree of correlation between a particular reference microphone and the primary microphone. It also provides an indication of the differential delay between a noise process observed from the primary and from a reference microphone.

Figures 4.7 (for lower band) and 4.8 (for upper band) show that at $60 \mathrm{Km} / \mathrm{hr}$, various degrees of correlation exist between each reference microphone and the primary microphone. This is true for all driving conditions. It was found that this degree of correlation depends on the driving condition, the microphone location and the frequency band.

Another important criterion is the relative differential delay. When comparing the cross-correlation of the upper and lower band, if the same noise process occupies both 
Mic. \# 1 Under the Hood

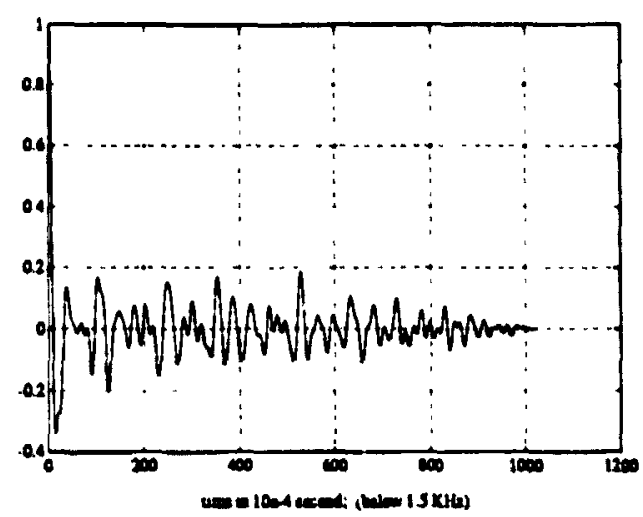

a.

Mic. \# 3 Under Floor Mat

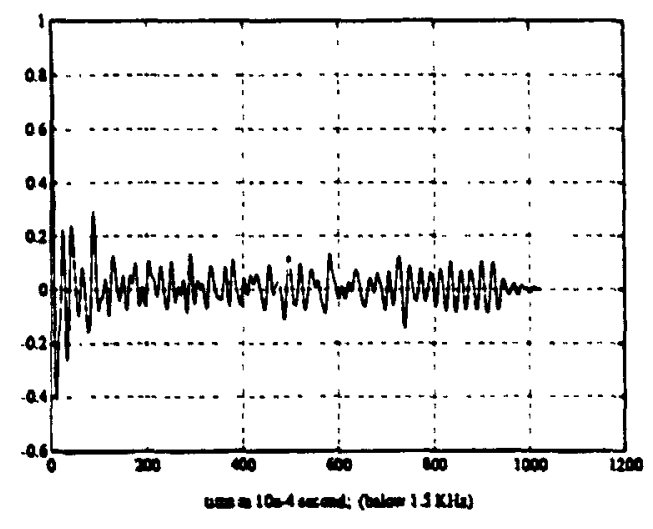

c.
Mic. \# 2 Under the Steering

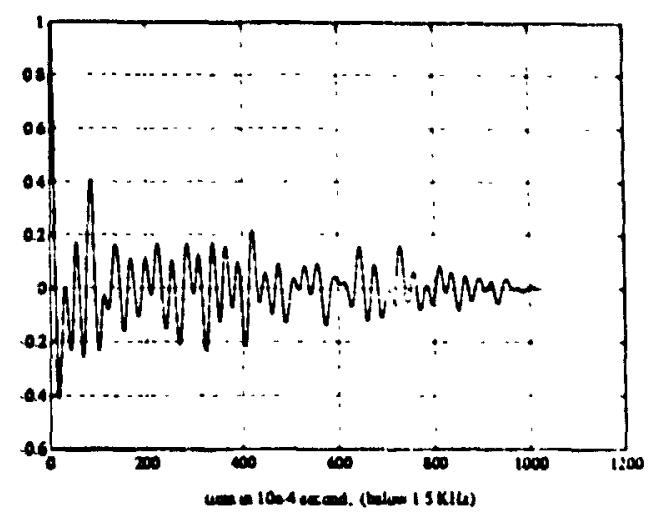

b.
Mic. \# 4 At Steering (Primary)

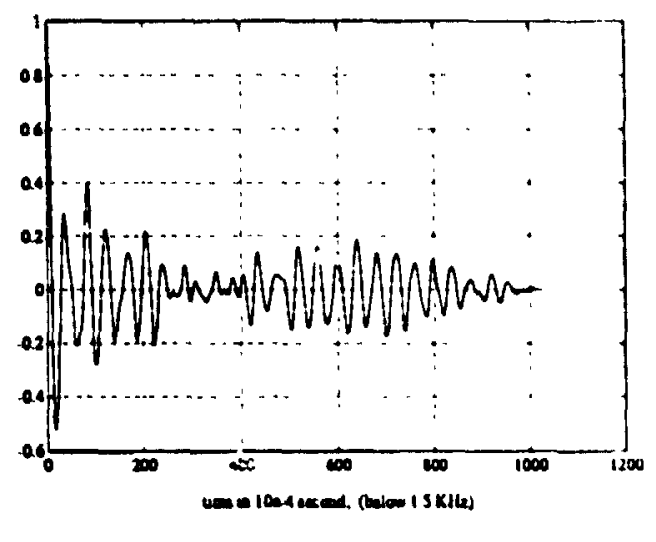

d.

Figure 4.5 Autocorrelation of the Lower Band (below $1.5 \mathrm{KHz}$ ), Engine Off, Fan On

bands then it should have the same differential delay in each band. As a guideline, the differential delay is normally obtained by locating the largest peak of the cross-correlation function. Foi example, if the cross-correlation between microphone \# 1 (under the hood) 
Mic. \# 1 Under the Hood

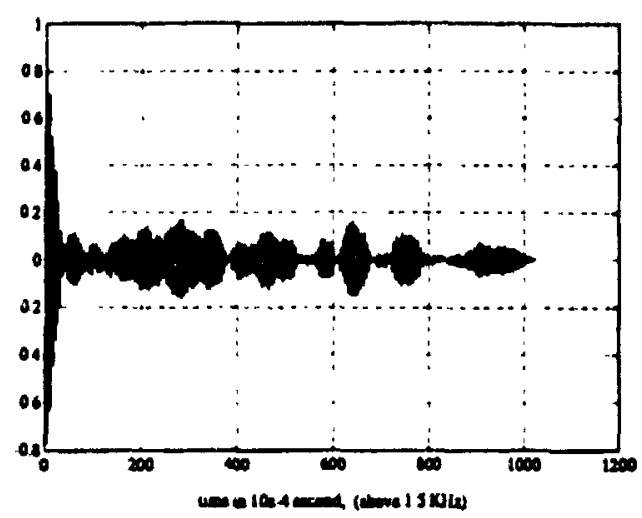

a.

Mic. \# 3 Under Flour Mat

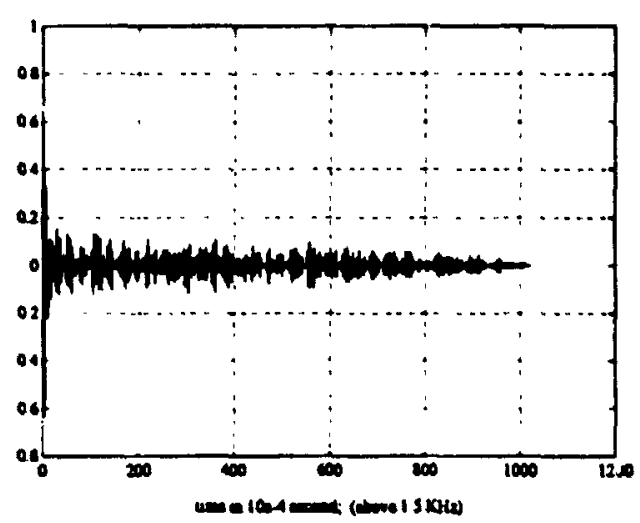

c.
Mic. \# 2 Under the Steering

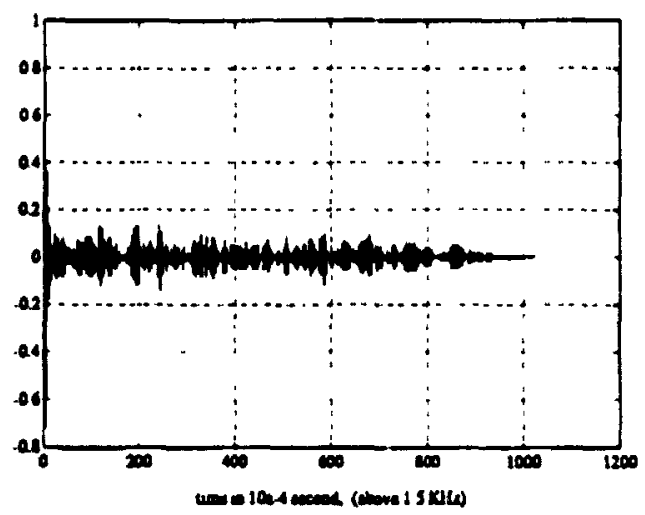

b.
Mic. \# 4 At Steering (Primary)

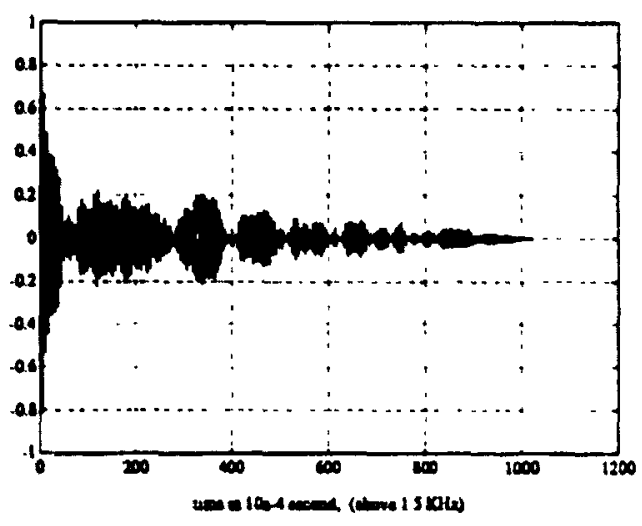

d.

Figure 4.6 Autocorrelation of the Upper Band (above 1.5 KHz), Engine Off, Fan On

and the primary microphone is considered when the car moves at $60 \mathrm{Km} / \mathrm{hr}$ (figures $4.7 . \mathrm{a}$ and 4.8.a), the lower band differential delay is about $20 \mathrm{msec}$ (figure 4.7.a) while it is about $0 \mathrm{msec}$ for the upper band (figure 4.8.a). This reinforces the fact that the noise 
Mic. \# 1 Under the Hond

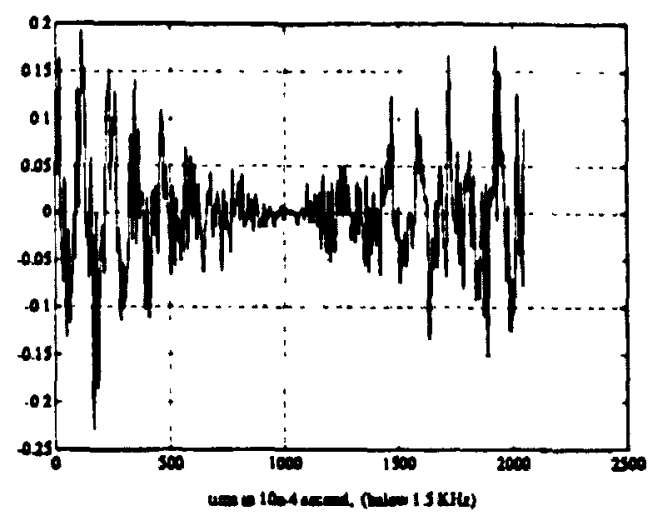

a.
Mic. \# 2 Under the Steering

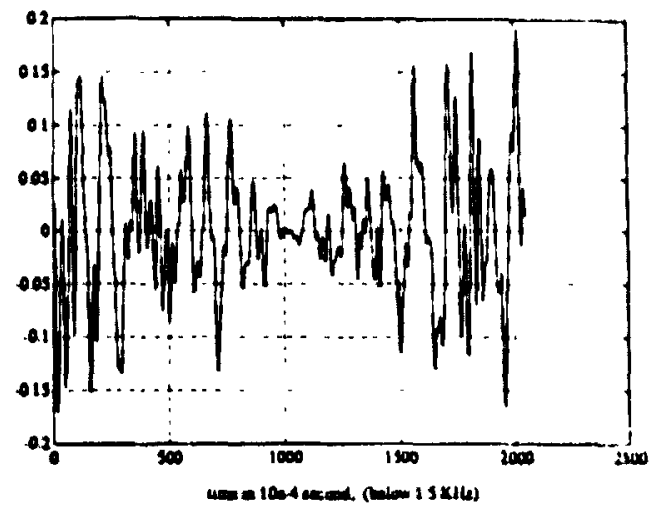

b.

Mic. \# 3 Under Floor Mat

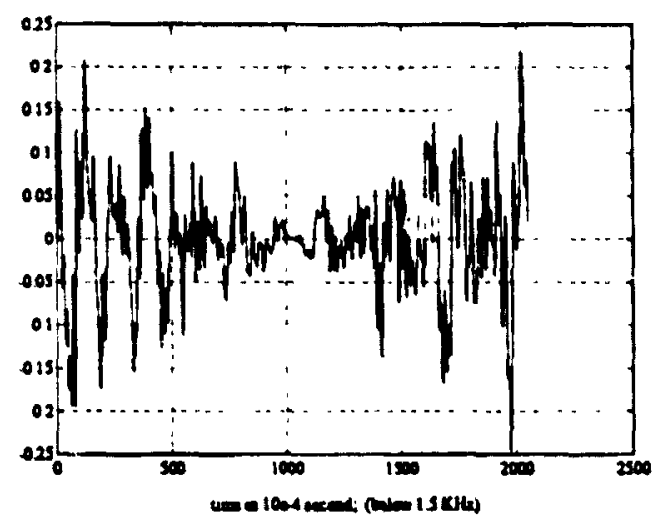

c.

Figure 4.7 Cross-Correlation Of the Lower Band (below $1.5 \mathrm{KHz}$ ) With Respect to the Primary Microphone (at the Steering Wheel); Car Speed $60 \mathrm{Km} / \mathrm{hr}$, Fan Off process observed in the upper band is different from that observed in the lower band.

Therefore, based on the frequency and the time domain analysis, it seems that various noise processes are dominant at different reference locations. Each one has a 
Mic. \# 1 Under the Hood

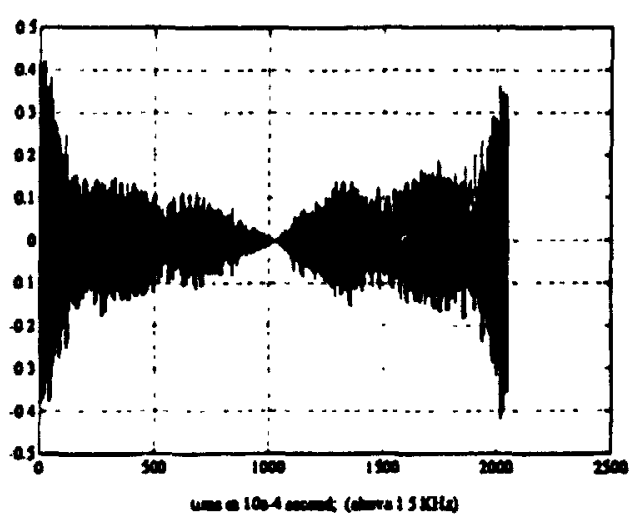

a.
Mic. \# 2 Under the Steering

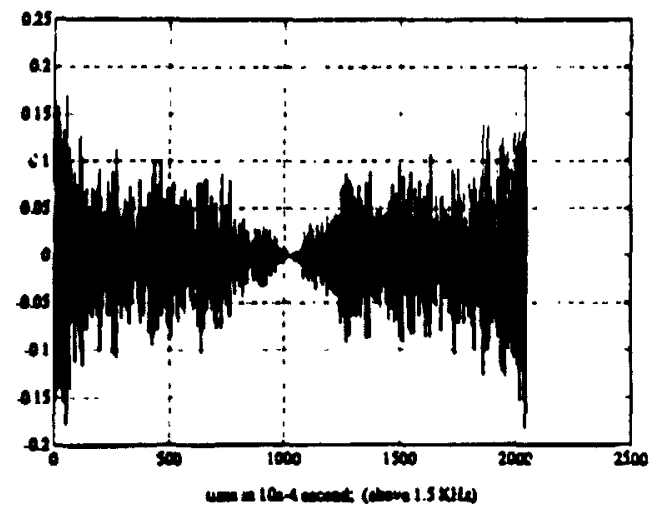

b.

Mic. \# 3 Under Floor Mat

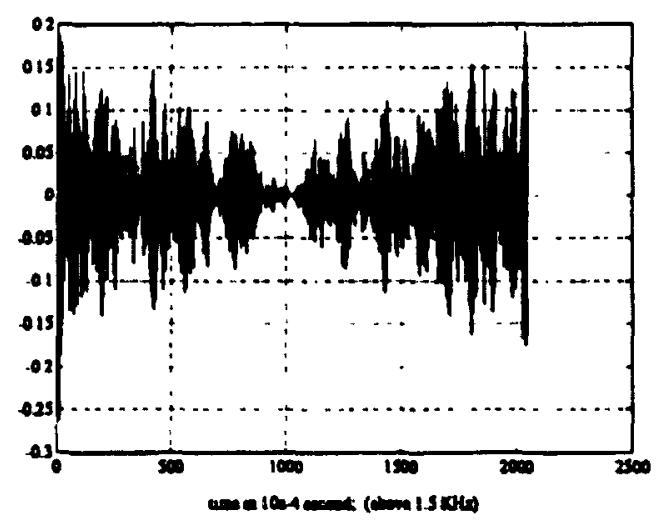

c.

Figure 4.8 Cross-Correlation Of the Upper Band (above $1.5 \mathrm{KHz}$ ) With Respect to the Primary Microphone (at the Steering Wheel); Car Speed $60 \mathrm{Km} / \mathrm{hr}$, Fan Cff

particular spectral signature, which depends on the driving conditions. Based on the data analysis, it is clear that a multiple reference microphone ANC could be implemented. it could make use of the best location for spatial filtering and the best spectral signature 
for temporal filtering. This is actually the beamformer (BF) approach which will be covered in chapter 6.

As a first step, independent adaptation should be implemented on each sub-band. This is called sub-band adaptation and it is covered in the next chapter. 


\section{Chapter 5 Two Microphone Technique for Noise Cancellation}

\subsection{Introduction}

In the previous chapter, background acoustic noise was analyzed. It revealec the existence of noise correlation between the primary microphone and the various secondary microphones. It was also found that the degree of correlation depends on the microphone location and the driving condition of the test. Based on the results analysis, some microphone configurations are proposed for a two microphone ANC.

In this chapter, various ANC are implemented using different microphone locations. The results are discussed in terms of the ANC efficiency and performance. The first part demonstrates how the transfer function linking the reference to the primary microphone relates to the noise model. It shows that the ANC performance relies on how accurate the transfer function estimation is. It also shows what perturbation factors affect the system. The second part shows the algorithm selection process. The third part discusses the results of implementing a selected algorithm on real data. The fourth part addresses an approach for solving the upper frequency band noise enhancement observe during tests and in the literature $[2,26,69]$. Finally the last part discusses some results of sub-band adaptation as well as their relation to the noise model. Guidance towards a multiple-microphone ANC configuration is also presented. 


\subsection{Transfer Function Estimation}

The ANC considered in this thesis is referred to as an identification system in the literature $[4,13,14]$ i.e. using the noise captured by a reference microphone, the system tries to estimate and extract the common noise from the primary. This is done by estimating the transfer function linking the reference to the primary microphone. Recall that this transfer function is assumed to be approximated by a FIR. The system performance relies on the degree of correlation between noise components in the (wo) microphones.

As shown in figure 5.1, the transfer function linking the secondary microphone to the primary can be expressed in terms of the observed signals $x(t)$ and $y(t)$. It is composed of $\mathrm{N}$ delays $\left(\tau_{1}\right)$. At the $\mathrm{i}^{\text {th }}$ stage, the reference signal $\mathrm{y}(t+\mathrm{i})$ is transformed by a factor $w_{1}$. This however provides no insight on how the system relates to the MIMO noise source system described in chapter 2 . Therefore the transfer function linking the reference microphone to the primary is more useful when expressed in terms of the noise sources.

Prior to developing an ANC, the model assumption should be recalled. It is assumed that all transfer functions are linear and that they can be approximated by a $N$ taps finite impulse response (FIR). Also, the approximation errors $e_{\mathbf{x}}(t)$ and $e_{\mathbf{y}}(t)$ of figure 2.4 are independent random processes with zero mean and variance $\sigma_{\mathrm{x}}{ }^{2}$ and $\sigma_{\mathrm{y}}{ }^{2}$. For ease of manipulation, it is assumed that no speech is active during adaptation. As pointed out in [6], if speech is present when adaptation takes place, it could result in reverberation or even speech cancellation. From figure 2.4 and equation (2.1), the observed noise process at the primary is

$$
x(t)=\underline{n}^{T}(t) \underline{C}(t)+e_{x}(t)
$$




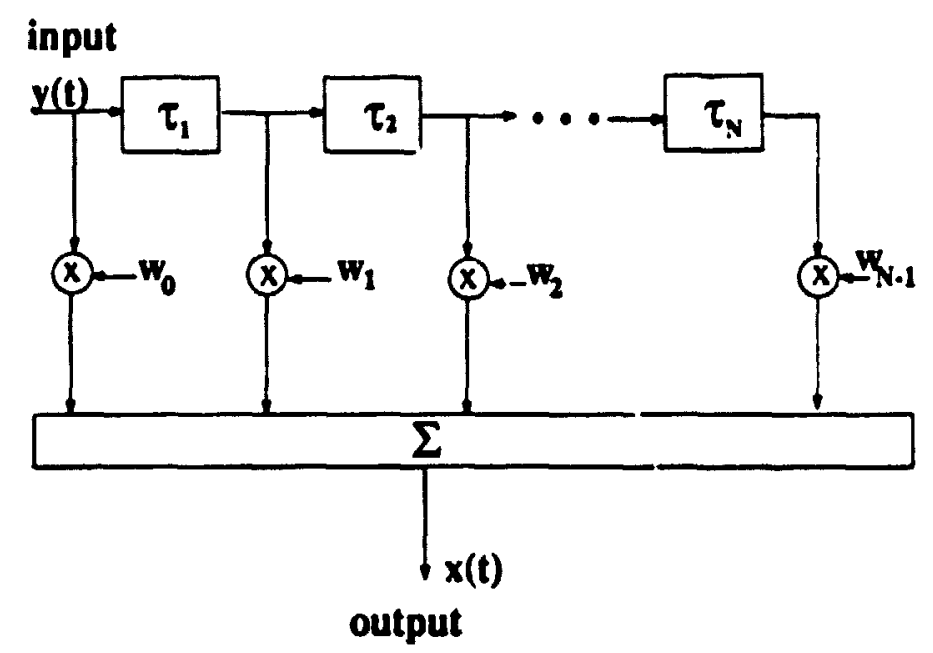

Figure 5.1 Transversal Filter With Variable Coefficient

At the reference microphone it is

$$
y(t)=\underline{n}^{T}(t) \underline{D}(t)+e_{y}(t)
$$

It is assumed that every transfer function in the system is limited to $\mathrm{N}$ taps. Therefore the transfer function linking the reference to the primary makes use of the $\mathrm{N}$ last observations of $y(t)$. Hence a $N \times 1$ vector representing $N$ last observation of $y(t)$ is denoted by

$$
\underline{\mathbf{y}}(\mathbf{t})=\underline{\mathbf{K}}(\mathbf{t})+\underline{\mathbf{e}}_{\mathbf{y}}(\mathbf{t})
$$


where

$$
\underline{K}(t)=\left[\begin{array}{c}
y(t) \\
y(t-1) \\
\cdot \\
\cdot \\
\cdot \\
y(t-N+1)
\end{array}\right]
$$

Substituting equation 2. in (5.4), equation (5.3) is linked to the noise processes

$$
\underline{K}(t)=\left[\begin{array}{c}
\underline{n}^{T}(t) \underline{D}(t) \\
\underline{n}^{T}(t-1) \underline{D}(t-1) \\
\cdot \\
\cdot \\
\cdot \\
\underline{n}^{T}(t-N+1) \underline{D}(t-N+1)
\end{array}\right]
$$

Since the system is approximated as being linear, a linear regression method as an estimation tool can be employed [4,8,13,14,58-61]. The process consists in approximating $x(t)$ using $\mathbf{y}(t)$ and a $N$ taps transversal filter $\underline{W}(t)$. Hence the estimation is given by

$$
x(t)=\underline{y}^{T}(t) \underline{W}(t)
$$

Figure 5.1 shows a transversal filter. The input goes through N-1 delays. Each tap coefficient of $\underline{W}(t)$ are adjusted in such a way that the mean square error is minimized. The error is the difference between the primary observation $\mathbf{x}(t)$ and its estimate $\overline{\mathbf{x}}(t)$.

$$
e(t)=x(t)-\bar{x}(t)
$$

By inspection of equation 5.7, the square error can only be positive. Implicitly taking the error expectation also gives a positive mean square error (mse). It can be seen as an upward cup on which surface lies the mean square error. 


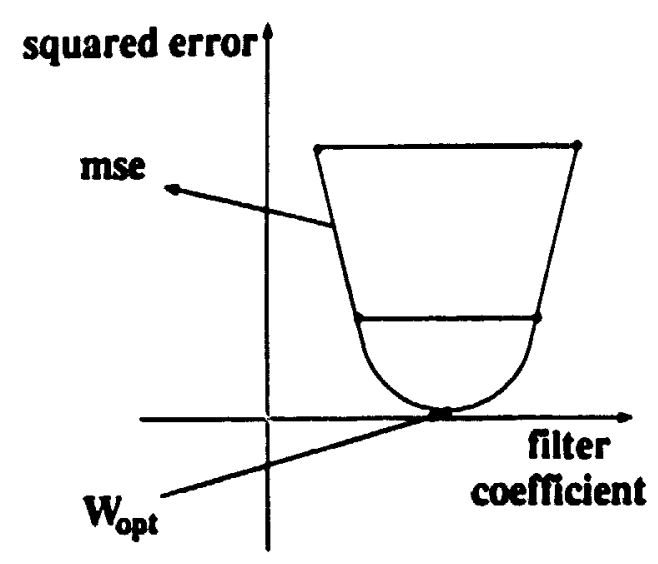

Figure 5.2 Mean Square Error Distribution With Respect to W(t)

Figure 5.2 illustrates the process for a one tap filter. Hence, there is a minimum mse, it is at the cup bottom. This point coincides with $\mathbf{W}_{\text {opt }}(t)$. The mse is given by taking the expectation of the square error, from equation (5.7) it is

$$
\text { mse }=E\left[e^{2}(t)\right]=E\left[x^{2}(t)-2 x(t) \tilde{x}(t)+\dot{x}^{2}(t)\right]
$$

Assuming that the transfer functions are constant over the observation period. The time dependence can be dropped since the expectation operator does not affect them. Substituting (5.1), (5.2), (5.3) and (5.6) in (5.8) and taking out of the expectation operator the constant terms gives

$$
\begin{aligned}
& \text { mse }=\underline{C}^{\mathrm{T}} \mathrm{E}\left[\underline{\underline{n}}(\mathrm{t}) \underline{\mathbf{n}}^{\mathrm{T}}(\mathrm{t})\right] \underline{\mathrm{C}}+2 \underline{\mathrm{C}}^{\mathrm{T}} \mathrm{E}\left[\underline{\mathrm{n}}^{\mathrm{T}}(\mathrm{t}) \mathrm{e}_{\mathbf{x}}(\mathrm{t})\right] \\
& +E\left[e_{x}^{2}(t)\right]-2 \underline{C}^{T} E\left[\underline{n}^{T}(t) \underline{K}^{T}(t)\right] \underline{w} \\
& -2 E\left[e_{x}(t) \underline{K}^{T}(t)\right] \underline{w}-2 \underline{C E}\left[\underline{n}^{T}(t) e_{y}(t)\right] \underline{w} \\
& -2 E\left[e_{x}(t) \underline{e}_{y}(t)\right] \underline{w}+\underline{W}^{T} E\left[\underline{K}(t) \underline{K}^{T}(t)\right] \underline{w} \\
& +2 \underline{W}^{T} E\left[\underline{e}_{y}(t) \underline{K}^{T}(t)\right] \underline{w}+\underline{w}^{T} E\left[\underline{e}_{y}(t) \underline{e y y}^{T}(t)\right] \underline{W}
\end{aligned}
$$


Using the independence properties of $e_{y}(t)$ and $e_{x}(t),(5.9)$ becomes

$$
\begin{aligned}
& \text { mse }=\underline{C}^{\mathrm{T}} \mathrm{E}\left[\underline{\mathrm{n}}(\mathrm{t}) \underline{\mathbf{n}}^{\mathrm{T}}(\mathrm{t})\right] \underline{\mathrm{C}}+\sigma_{\mathrm{ex}}^{2}-2 \underline{C}^{\mathrm{T}} \mathrm{E}\left[\underline{\mathrm{n}}(\mathrm{t}) \underline{\underline{K}}^{\mathrm{T}}(\mathrm{t})\right] \underline{\mathrm{W}} \\
& +\underline{W}^{T} E\left[\underline{K}(t) \underline{K}^{T}(t)\right] \underline{w}+\underline{w}^{T} E\left[\underline{e y y}(t) \underline{e y}^{T}(t)\right] \underline{W} \\
& =\underline{C}^{\mathrm{T}} \mathbf{R}_{\mathrm{n}} \underline{\mathbf{C}}+\sigma_{\mathrm{ex}}^{2}-2 \underline{\mathbf{C}}^{\mathrm{T}} \underline{\mathrm{p}}_{\mathrm{n}} \underline{\mathbf{W}}+\underline{\mathbf{w}}^{\mathrm{T}} \mathbf{R}_{\mathrm{K}} \underline{\mathrm{W}}+\underline{\mathbf{w}}^{\mathrm{T}} \mathbf{R}_{\mathrm{ey}} \underline{\mathrm{W}}
\end{aligned}
$$

where $\mathbf{R}_{\mathbf{n}}$ is the autocorrelation of the noise source vector $\underline{n}(t)$.

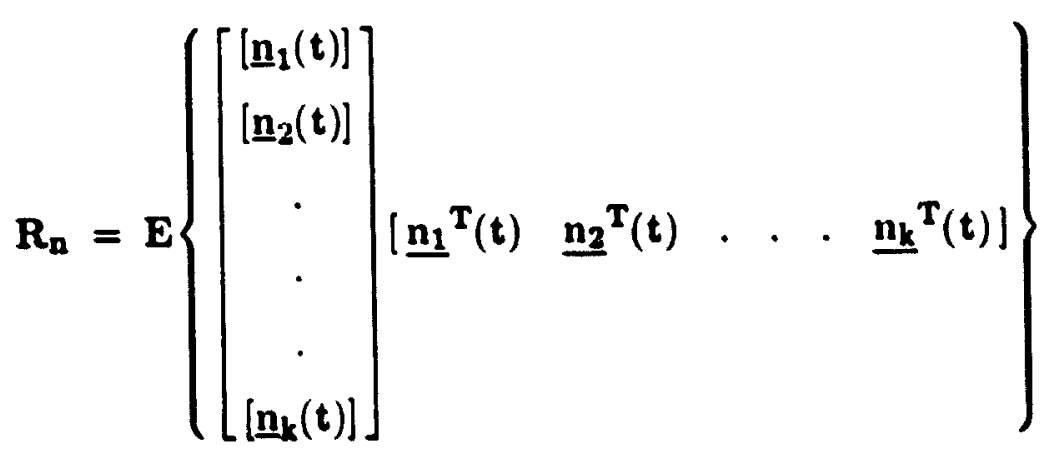

It was assumed that each noise source have zero mean and are independent of each other hence

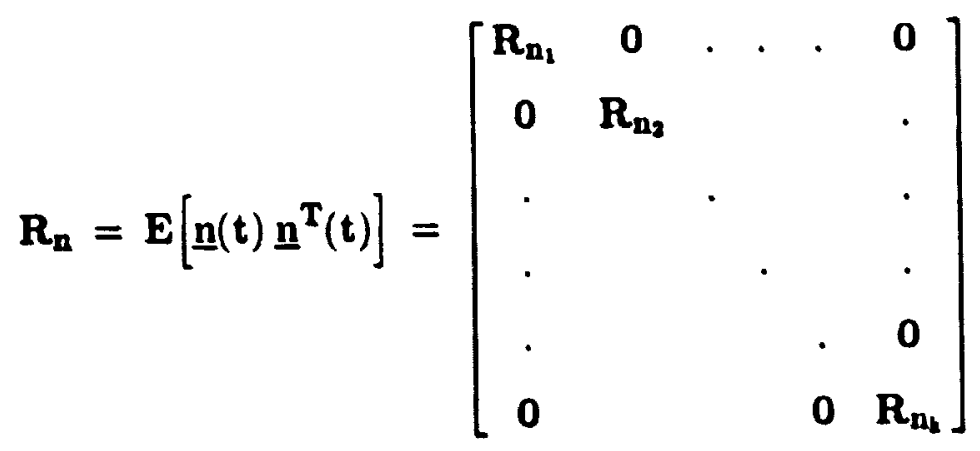


where

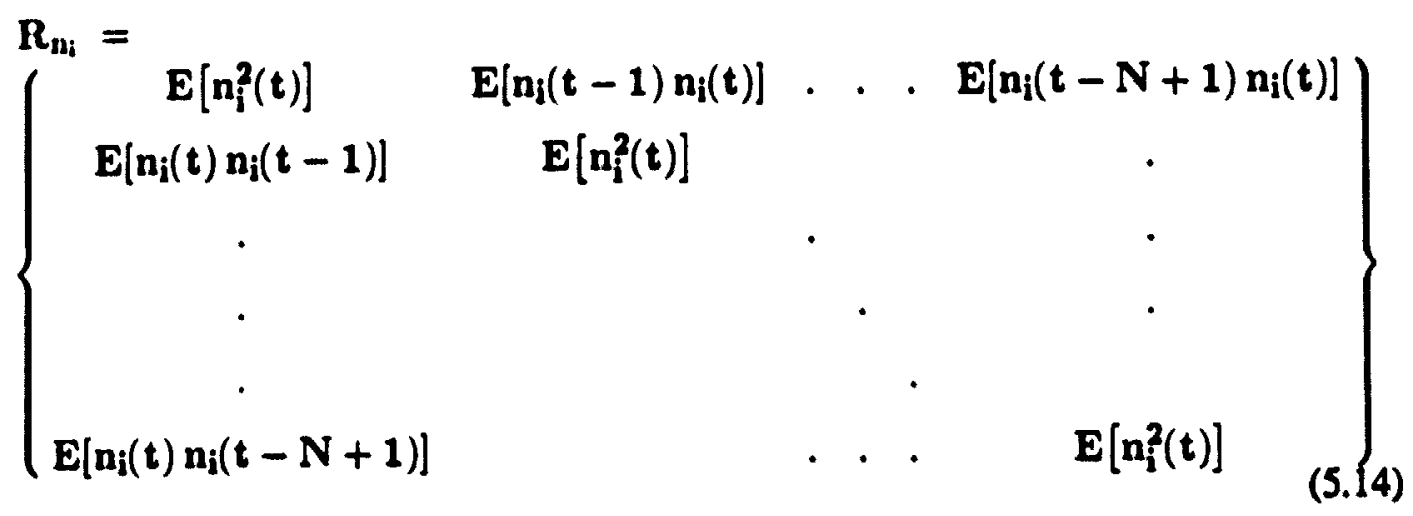

$\mathbf{R}_{\mathbf{n}_{\mathbf{i}}}$ is toeplitz and symmetric with respect to the main diagonal

$\mathbf{R}_{\mathbf{K}}$ is the autocorrelation of the noise sources filtered by $\underline{\mathbf{D}}$

$$
\mathbf{R}_{\mathbf{K}}=\mathbf{E}\left[\underline{\mathbf{K}}(\mathbf{t}) \underline{\mathbf{K}}^{\mathbf{T}}(\mathbf{t})\right]
$$

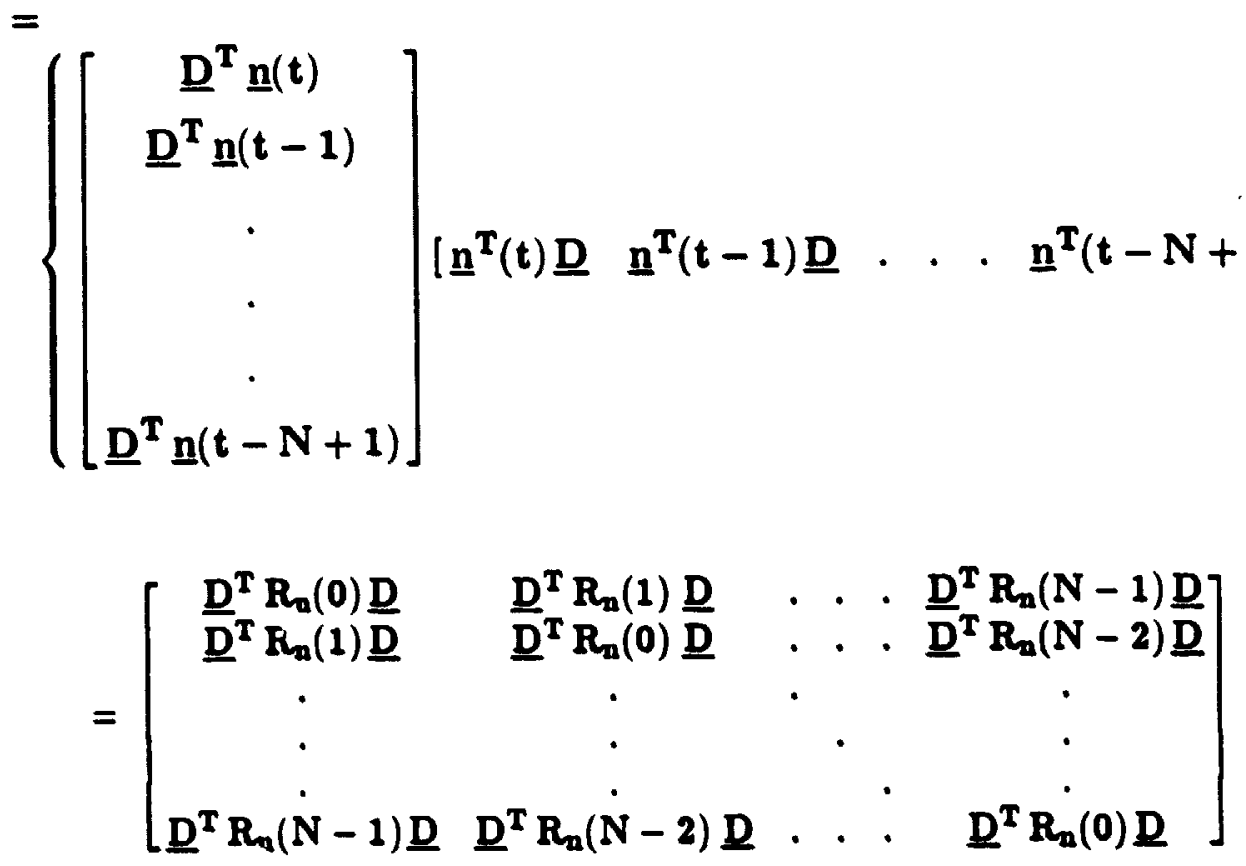


where

$$
R_{n_{i}}=E\left[\underline{n}(t) \underline{\underline{n}}^{T}(t-i)\right]
$$

the cross-correlation becomes

$$
\begin{aligned}
& \underline{p}_{n}=E\left[\underline{C}^{T} \underline{n}(t) \underline{K}^{T}(t)\right] \\
& =E\left\{\underline{C}^{T} \underline{n}(t)\left\{\underline{n}^{T}(t) \underline{D} \underline{n}^{T}(t-1) \underline{D} \cdot . \cdot \underline{n}^{T}(t-N+1) \underline{D}\right]\right\} \quad \because 20 ! \\
& \underline{\underline{p}}_{\mathbf{n}}=\left[\begin{array}{c}
\underline{C}^{T} R_{n}(0) \underline{D} \\
\underline{C}^{T} R_{n}(1) \underline{D} \\
\cdot \\
\cdot \\
\cdot \\
\underline{C}^{T} R_{n}(N-1) \underline{D}
\end{array}\right]
\end{aligned}
$$

$\mathbf{R}_{\mathrm{ey}}$ is the autocorrelation of $\mathbf{e}_{\mathbf{y}}(\mathrm{l}$ !

$$
\mathbf{R}_{\text {ey }}=\mathbf{E}\left[\underline{\underline{e}}_{\mathbf{y}}(\mathrm{t}) \underline{\underline{e}}_{\mathbf{y}}^{\mathbf{T}}(\mathbf{t})\right]=\sigma_{\mathrm{ey}}^{2} \mathbf{I}
$$

where $I$ is an identity mat : 8 of dimension $N \times N$ and $\sigma_{\text {ey }}^{2}$ is the variance of $1, ;$ il.

By inspecting figure 5.2, the minimum mse can be obtained by taking the 1.:vative of equation (5.11) with respect to $\underline{W}$ and setting it to zero. This provides the pirnum value of $\underline{\mathbf{W}}$.

$$
\frac{\partial m s e}{\partial \underline{W}}=-2 \underline{p}_{n}+2 R_{K} \underline{W}_{\text {opt }}=0
$$




$$
\underline{\mathbf{p}}_{\mathbf{n}}=\left(\mathbf{R}_{\mathrm{K}}+\mathbf{R}_{\mathrm{ey}}\right) \underline{\mathbf{W}}_{\mathrm{opt}}
$$

This is the well known Wiener-Hopf equation [14]. By inspection $\mathbf{R}_{\mathrm{ey}}$ is a perturbation factor. It limits the adaptation or $\mathbf{W}$ 's estimation b' introducing a bias. This bias can be viewed as a modification of the system modes [14,67] by perturbing the main diagonal of $\mathbf{R}_{\mathbf{K}}$. Regardless of $\mathbf{R}_{\mathbf{e y}}$, it is obvious that the optimum filter cocfficients depend on $\mathbf{R}_{\mathbf{K}}$ only. This is so since the aim of the ANC is to make use of the common noise (i.e. correlated noise) to both the primary and reference microphones. Recall that the error $e_{y}(t)$ comes from two sources of approximation. First from the nonlinear part of the system and second from the selection of a too small number of i aps which in fact could be larger. Based on the system's definition, it would be fair to assume that $e_{y}(t)$ is negligeatle. Hence equation (5.19) becomes

$$
\underline{\mathbf{p}}_{\mathrm{n}} \approx \mathbf{R}_{\mathrm{K}} \underline{\mathbf{W}}_{\mathrm{opt}}
$$

hence

$$
\underline{\mathrm{W}}_{\mathrm{opt}} \approx \mathrm{R}_{\mathrm{K}}^{-1} \underline{\mathrm{p}}_{\mathrm{n}}
$$

Companng $\mathbf{p}_{n}$ and $\mathbf{R}_{K}$ using equations (5.14) and (5.16), $\because$ is evident that acts on each row of $\mathbf{R}_{\mathbf{K}}$ for completing the gap existing between $\underline{\mathbf{D}}$ and $\underline{\mathbf{C}}$. Hence $\underline{\mathbf{W}}$ makes use of tie $N$ last reference observations $y(t)$ for estimating $x(t)$ the primary output.

There are some system constraints for allowing $\underline{\mathbf{W}}$ to exist. First, the system must be causal i.e. the delay stemming from $\underline{\mathbf{D}}$ must be smaller than the one stemming from $\underline{\mathrm{C}}[2,69]$. This can be compensated by inserting a delay at the primary ourput prior to its processing $[1,2,69]$. Second, the error $e_{y}(t)$ must be small enough so that the bias factor does not deteriorate the ANC efficiency to a large extent. Third, $\underline{\mathbf{C}}$ a:d $\underline{\mathbf{D}}$ must be approximately FIRs of length $\mathrm{N}$ at the most. 


\subsection{Algorithm}

In this section, a least square (LS) algorithm is selected for implementing an ANC. The selection is based on :

(i) tracking capability to a time varyiant system;

(ii) ease of implementation;

(iii) reliability; and

(iv) the end users operating the system.

Three algorithm are considered: the recursive least square (RLS), the block least square (BLS) and, the stochastic gradient or least mean square (LMS).

The RLS algorithm converges very fast in a non-noisy envieonment. However, it has been shown that in a noisy environment its convergence is about the same as that of the LMS [14]. The algorithm requires a high computational capability for inverting the matrix $\mathbf{R}_{\mathbf{K}}$ of equation (5.21). Also it is a well known fact that the RLS is bound to become unstable after an unpredictable time of operation. A recovery system must therefore be implemented as a compensator. Hence, the RLS is discarded since it is unreliable and it costs too much in compution requirement.

The BLS takes a block of data and finds the optimum transfer function for it [13]. At first glance, this looks appealing since it would allow to implement an optimum coding like approach ANC. The Karhunen-Loeve Transform (KLT) could well be used with the algorithm. The aim would be to establish which dominant modes are common in the primary and reference microphones. Then a simple subtraction of it could be done from the primary signal. Preliminary tests have shown that only a few modes compose each noise signal. However, the task of comparing the modes became soon too complex. Also the computational requirement for this approach seemed too much 
for a real time system. It was therefore decided to disregard the KLT but to bare in mind a sub-optimum approach using the frequency domain.

The BLS method could well have been implemented using a frequency domain technique but it was too expensive in computation time. It was decided to consider the simple BLS algorithm. A problem arises when using this algorithm. It is as demanding as the RLS since it too must carry on a matrix inversion in order to find the optimum coefficients set $\underline{W}$. Also since each block are processed independently from one to another, there sometimes is no smooth transition between them. The sudden transitions become annoying to the end user [13].

The BLS was discarded in a early stage on the ground that it is too expensive to implement and it annoys the end user.

This leaves the LMS algorithm. It is a well known algorithm in the literature and has proven to be reliable in a slowly varying environment $[8,11,13,14]$. This algorithm adapts to the system characteristics in an intuitive way. It uses the instantaneous gradient [e(t) $\mathbf{y}(t)]$ with respect to the transfer function $\underline{\mathbf{W}}(\mathrm{t})$. Using equations (5.6) and (5.7) in the well known LMS algorithm $[2,8,13,14]$ the following equations system is obtained

$$
\underline{\mathbf{W}}(\mathbf{t}+\mathbf{1})=\underline{\mathbf{W}}(\mathbf{t})+\beta(\mathbf{t}) \mathbf{e}(\mathbf{t}) \underline{\mathbf{y}}(\mathbf{t})
$$

where $\underline{W}(t+1):$ is the new FIR coefficients;

$\underline{W}(t)$ : is the old FIR coefficient

$3(t)$ is a variable step size normalized to the summation of the total instantaneous power of the observed sequence $\mathbf{y}(t)$. This allows the step size to adapt to the system characteristics variation. Preliminary testing have proven that a fixed step size always lead to an unstable ANC. From $[5,15]$, it is shown that for algorithm convergence, the 
step size niust be bounded by

$$
0<\beta(t)<\frac{1}{\lambda_{\max }}
$$

$\lambda_{\max }$ is the largest eigenvalue of $\mathbf{R}_{\mathbf{K}}$, the instantaneous autocorrelation of $\mathbf{g}(\mathbf{t})$. It is known that the eigenvalues distribution represent the system modes distribution $[14,67]$. Informal testing has shown that there is only a few dominant modes i.e. eigenvalues in the system. Also from [14], $\lambda_{\max }$ is upper bounded by trace of $\mathbf{R}_{\mathbf{K}}$.

$$
\lambda_{\max } \leq \operatorname{trace}\left(\mathbf{R}_{\mathbf{K}}\right)
$$

Further to this, in [14], it is shown that for the instantaneous autocorrelation,

$$
\operatorname{trace}\left(R_{K}\right)=\underline{y}^{T}(t) \underline{y}(t)
$$

hence 5.24 becomes bounded by

$$
\lambda_{\max } \leq \underline{\mathbf{y}}^{\mathbf{T}}(\mathbf{t}) \underline{\mathbf{y}}(\mathbf{t})
$$

In [15], $\beta(t)$ is defined in such a way that it is upper bounded by

$$
\beta(t)=\frac{\mathbf{a}_{\mathbf{i}}}{\left[\underline{\mathbf{y}}^{\mathrm{T}}(\mathbf{t}) \underline{\mathbf{y}}(\mathbf{t})+\mathbf{b}_{\mathbf{i}}\right]}
$$

where $a_{1}$ and $b_{1}$ are some limiting constants limiting $\beta(t)$ to a finite value. This prevents from having a very large $\beta(t)$ when the norm of $\mathbf{y}(t)$ is close to zero.

The LMS algorithm is implemented with a set to 1. This provides the fastest convergence rate hence the best tracking capability [15].

\subsection{Results Of a Two Microphone ANC}

The LMS algorithm described in the previous section was implemented on a SUN and PC workstations using the MATLAB package. The car data described in chapter 
3 is disposed in files of 53248 samples. For each file, the mean is removed prior to any processing. The system efficiency is measured in terms of the instantaneous power of the input and output signal of the ANC. Note that this only provides a qualitative measure of the system efficiency. The efficiency is given by

$$
\text { ef }=10 \log _{10}\left[\frac{\underline{e}^{T}(t) \underline{e}(t)}{\underline{x}^{T}(t) \underline{x}(t)}\right] d B
$$

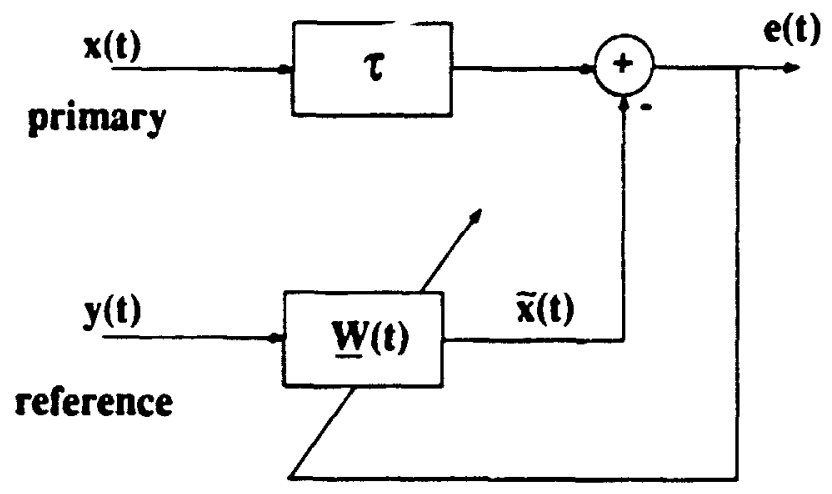

Figure 5.3 Two Microphone ANC Configuration

The ANC configuration is shown in figure 5.3. A delay is added at the primary in order to compensate for the delay stemming from the adaptive filter $\underline{W}(t)$. This ensure system causality. The PSD of the ANC output signal $(e(t))$ and the input signals $(x(t))$ are plotted in a logarithmic scale. Since the estimated signal $(\tilde{x}(t))$ is subtracted from the primary signal $(x(t))$ as shown in figure $5.3, a 0 d B$ improvement is the superposition of the two curves. Hence, if the output signal PSD is below that of the primary signal, then there has been a SNR improvement.

Figures 5.4-5.10 show the result of a Two MicrophoneANC. In every cases, good noise suppression takes place in the lower end of the acoustic band. The efficiency is about $10 \mathrm{~dB}$. However, in the upper band, there is noise enhancement. 
Mic. \# 1 Under the Hood

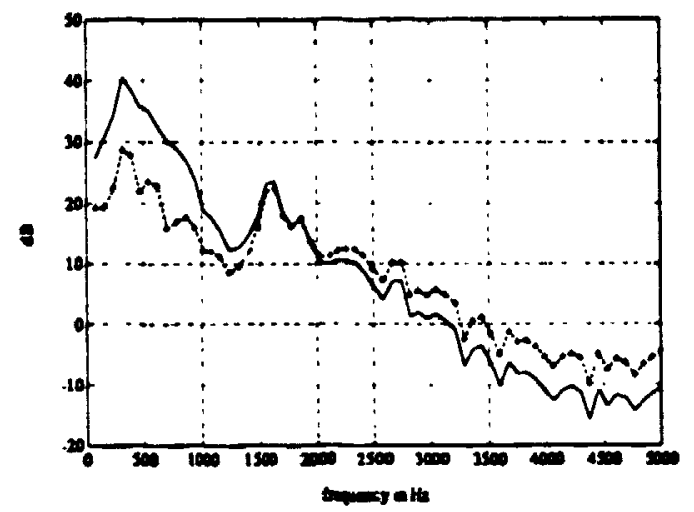

a.
Mic. \# 2 Under the Steering

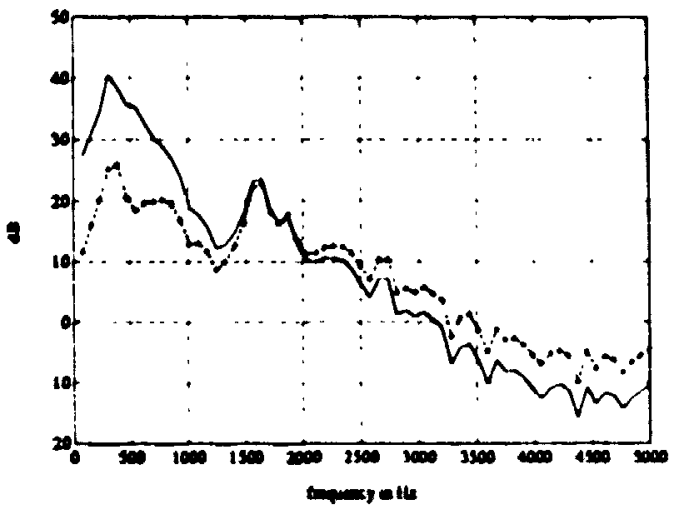

b.

Mic. \# 3 Under Floor Mat

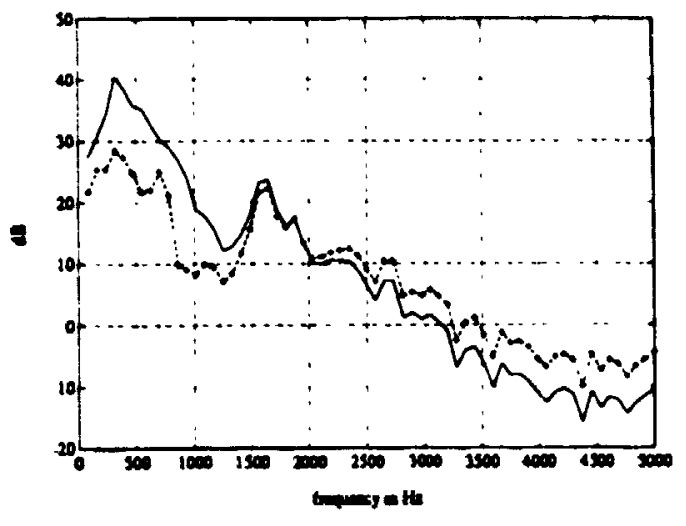

c.

Figure 5.4 Result Of a Two MicrophoneANC, Engine Off, Fan On; Output :-+-

There are some common observations which can be made with respect to each location. These observations hold for all driving conditions. In general, location \# 2 (under the steering wheel) performs better than the others for the frequency regions 
Mic. \# 1 Under the Hood

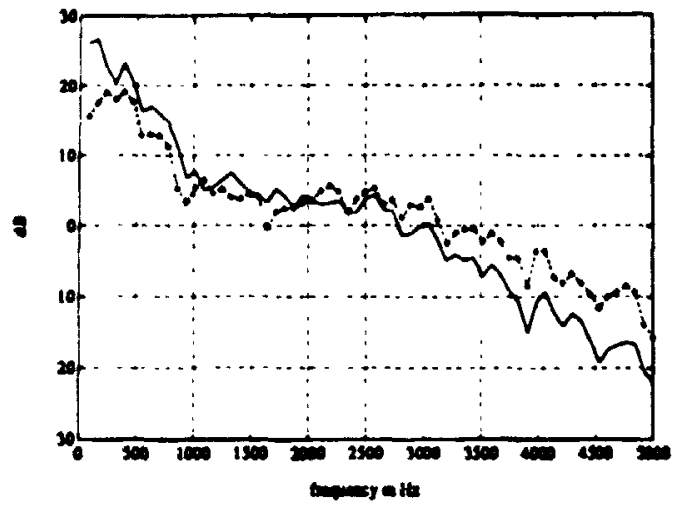

a.
Mic. \# 2 Under the Steering

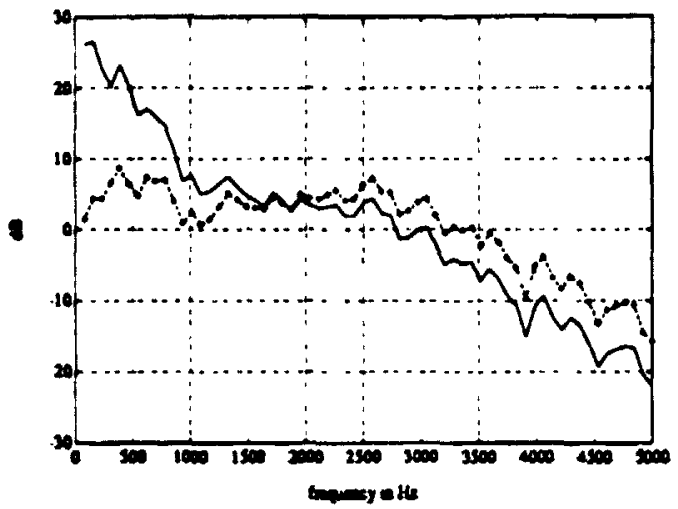

b.

Mic. \# 3 Under Floor Mat

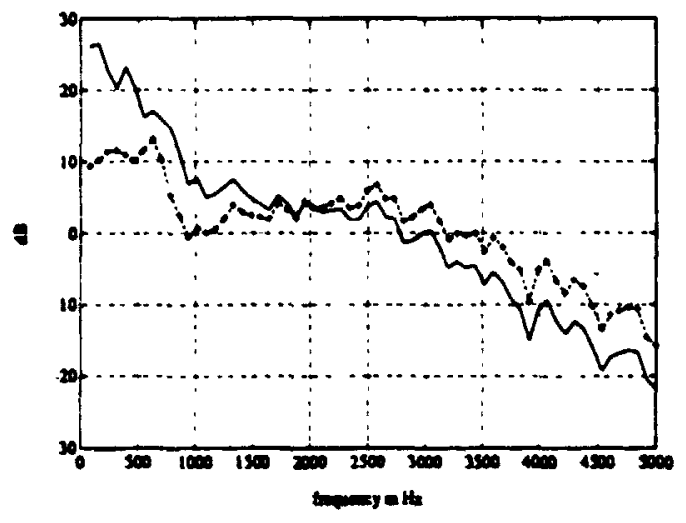

c.

Figure 5.5 Result Of a Two MicrophoneANC, Car Idling, Fan Off; Output :---

ranging from $0-800 \mathrm{~Hz}$. This can clearly be observed throughout every figures (5.4-5.10, $5.19-5.25)$.

Location \# 3 (under the floor mat) provides the best noise cancellation for the 
Mic. \# 1 Under the Hood

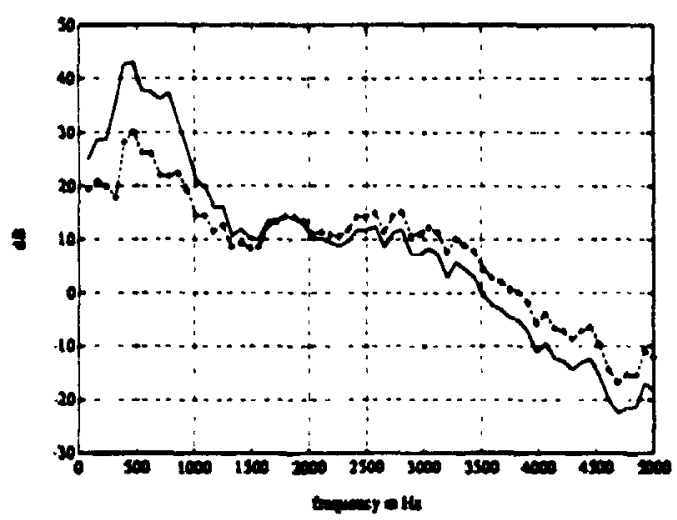

a.
Mic. \# 2 Under the Steering

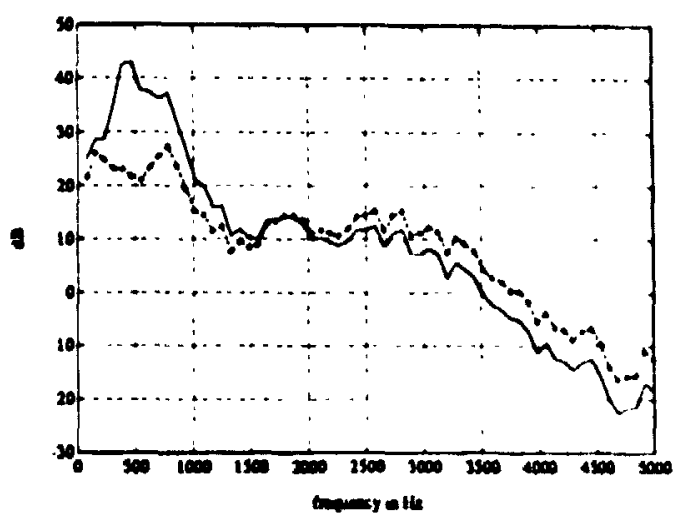

b.

Mic. \# 3 Under Floor Mat

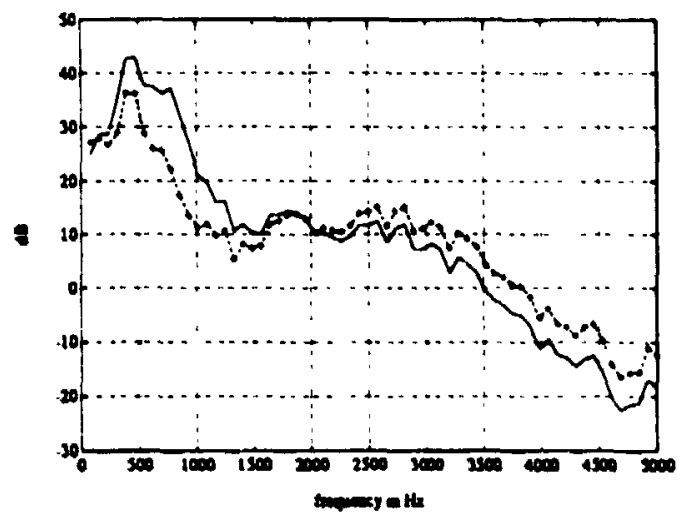

c.

Figure 5.6 Result Of a Two MicrophoneANC, Car Idling, Fan On; Output :-tfrequency region ranging from $800-1500 \mathrm{~Hz}$.

Location \# 1 (under the hood) seems to always be the one providing the least noise suppression. However, it provides a very important information with respect to the 
Mic. \# 1 Under the Hood

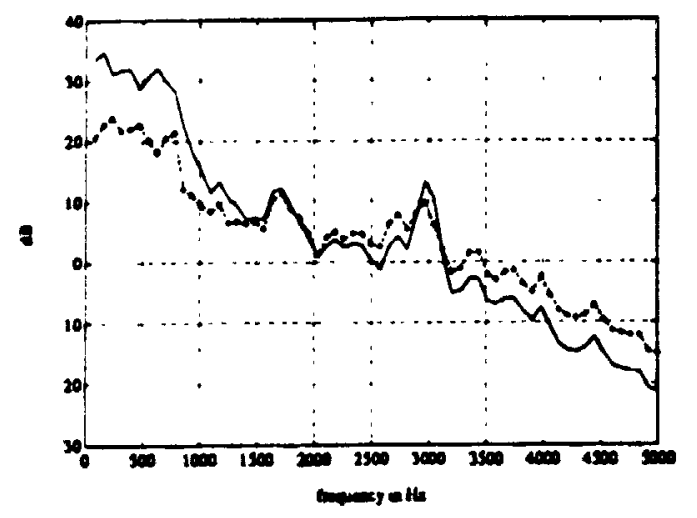

a.
Mic. \# 2 Under the Steering

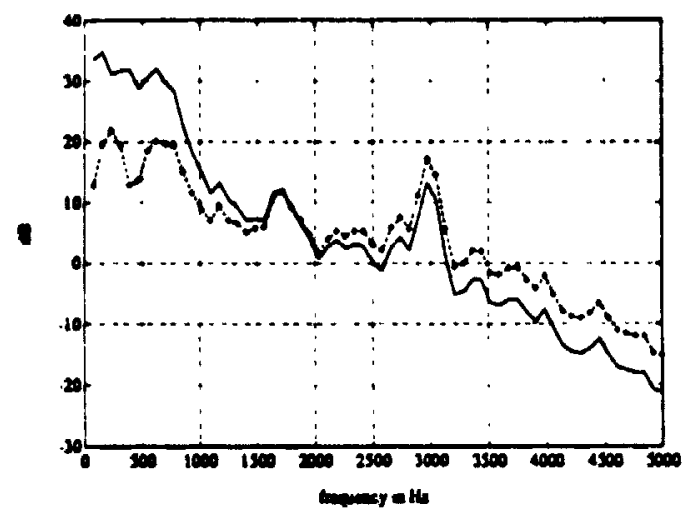

b.

Mic. \# 3 Under Floor Mat

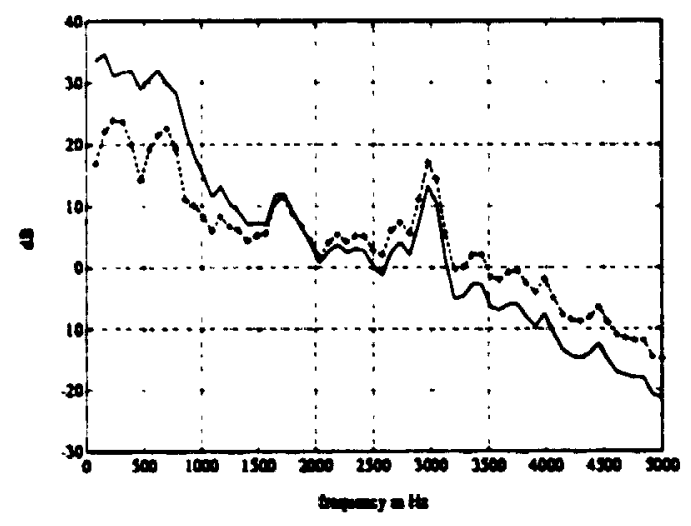

c.

Figure 5.7 Result Of a Two MicrophoneANC, Car Speed $60 \mathrm{Km} / \mathrm{hr}$, Fan Off; Output :-t-

noise model. In the upper band, the PSD analysis revealed that a peak in the upper band is common to both location \#1 and \#2. Figures 5.7.a and 5.8.a show a partial noise suppression of a peak located at $3000 \mathrm{~Hz}$. This indicates that noise suppression 
Mic. \# 1 Under the Hood

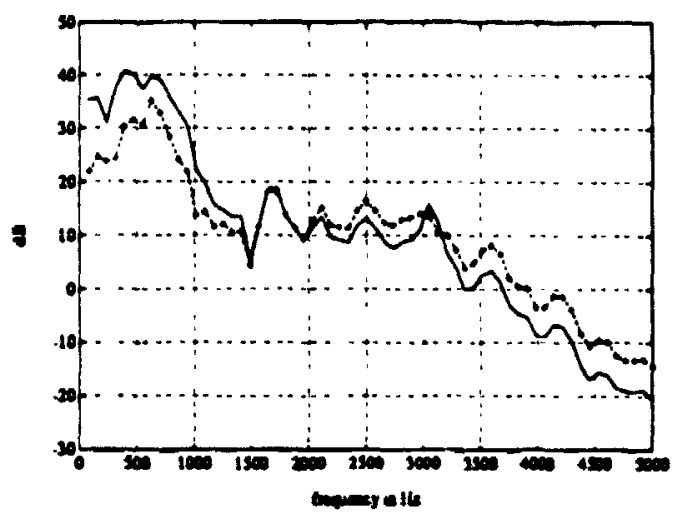

a.
Mic. \# 2 Under the Steering

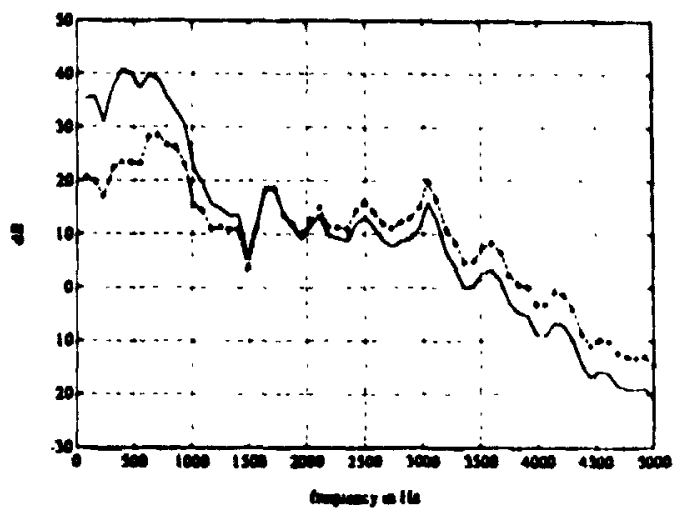

b.

Mic. \# 3 Under Floor Mat

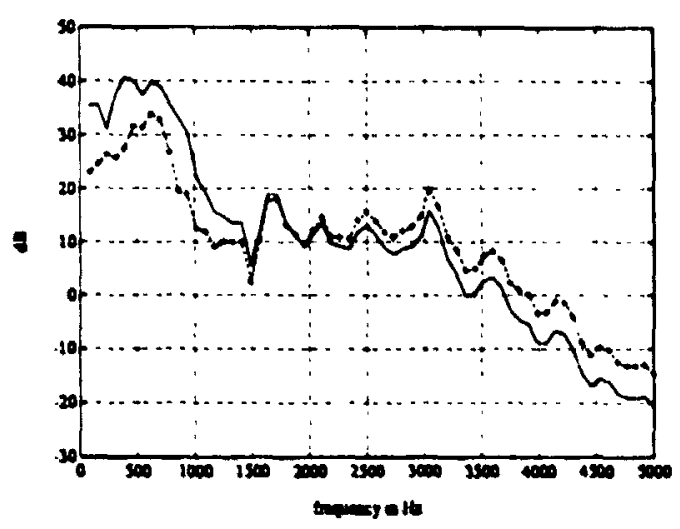

c.

Figure 5.8 Result Of a Two MicrophoneANC, Car Speed $60 \mathrm{Km} / \mathrm{hr}$, Fan On; Output :--in the upper band is possible. It also confirms the existence of correlated processes in the upper band as found in chapter 4 .

In chapter 4, it was indicated that different noise processes seem to occupy the upper 
Mic. \# 1 Under the Hood

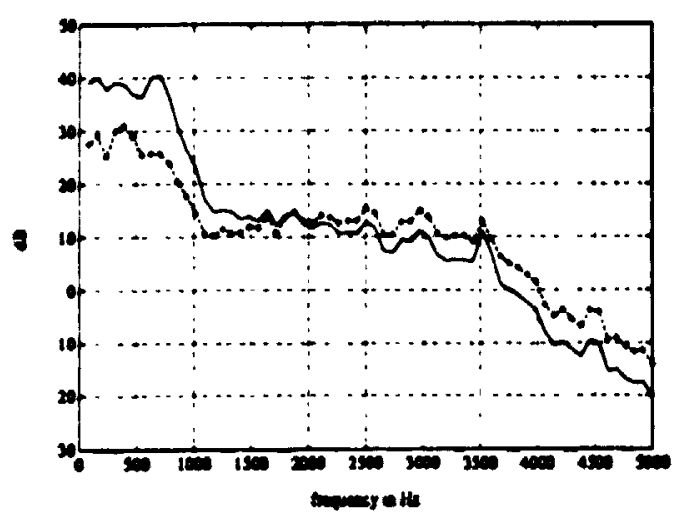

a.
Mic. \# 2 Under the Steering

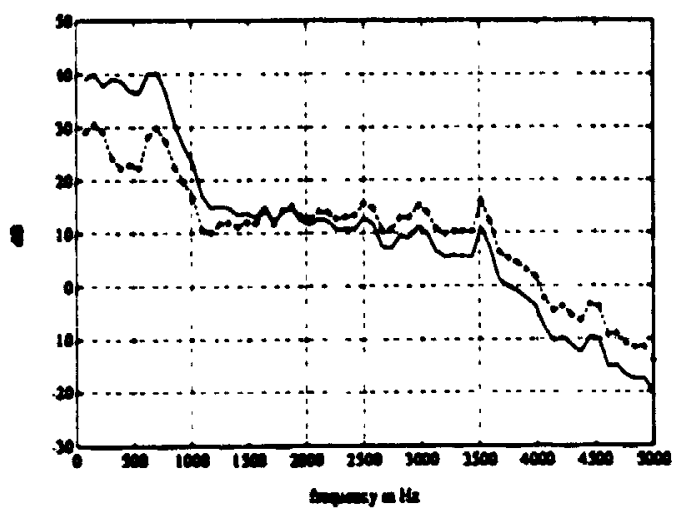

b.

Mic. \# 3 Under Floor Mat

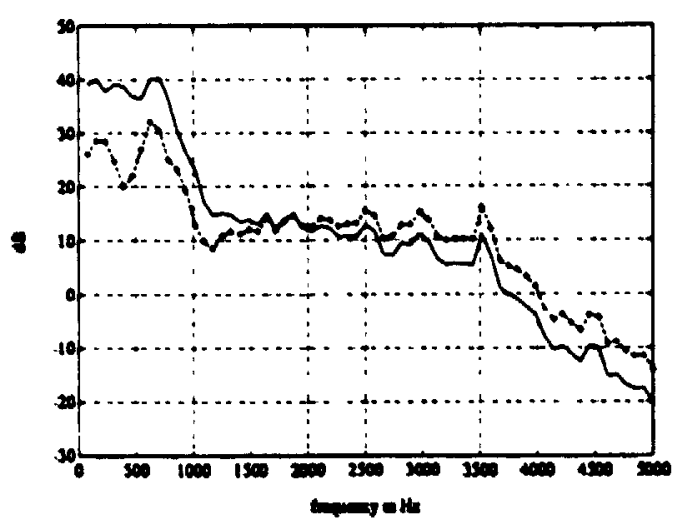

c.

Figure 5.9 Result Of a Two MicrophoneANC, Car Speed $100 \mathrm{Km} / \mathrm{hr}$, Fan Off; Output :-+-

and lower band. Also, it was shown that the two processes (from the upper and lower band respectively) did not share the same differential delay. A second approach, which 
Mic. \# 1 Under the Hood

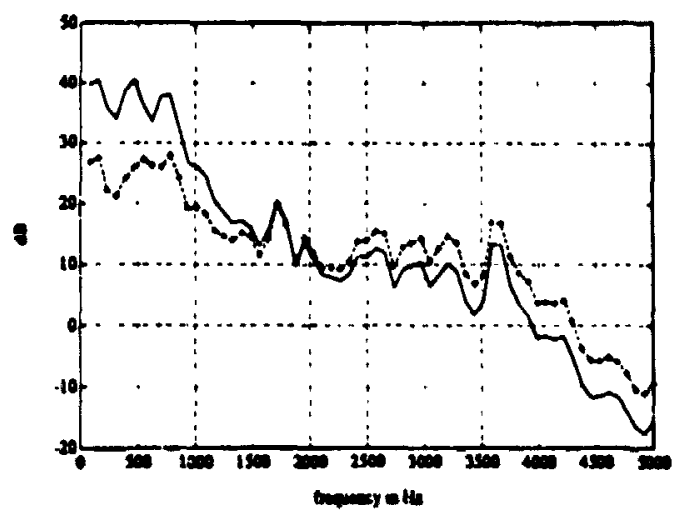

a.
Mic. \# 2 Under the Steering

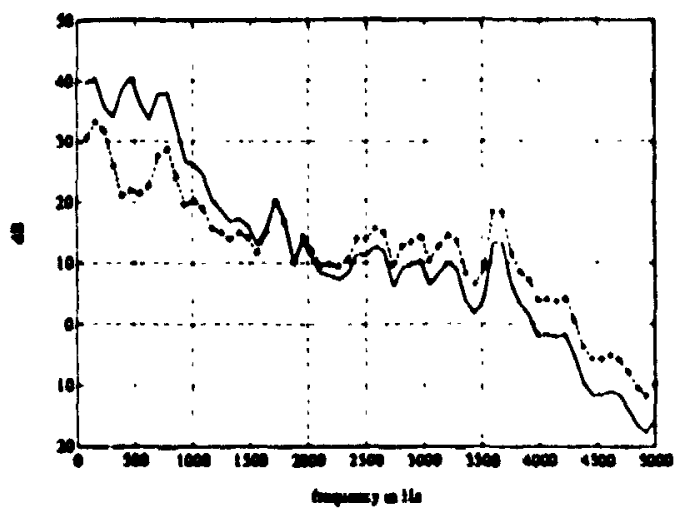

b.

Mic. \# 3 Under Floor Mat

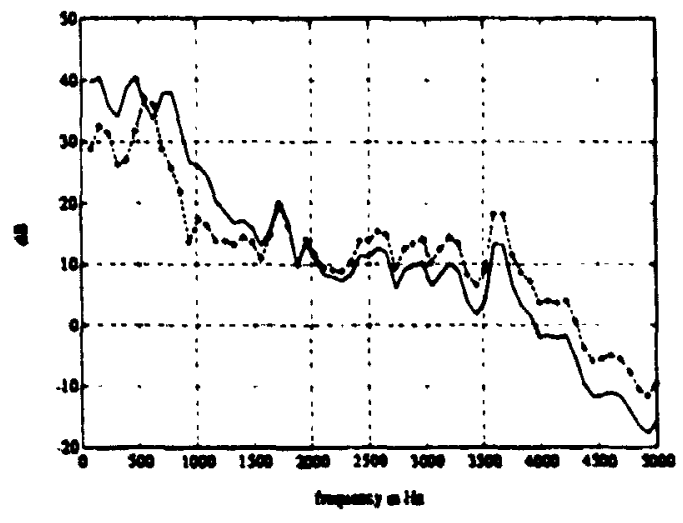

c.

Figure 5.10 Result Of a Two MicrophoneANC,

Car Speed $100 \mathrm{Km} / \mathrm{hr}$, Fan On; Output :-+-

minimizes the noise enhancement at high frequencies is the sub-band adaptation. 


\subsection{Sub-Band Adaptation}

Goubran et al. [1] and Ambruster et al. [20] show how a multi-band adaptation looks like from a MIMO noise system. While, in theory, the use of many sub-bands may seem appealing. This could well lead to a tone in band problem. This is a side effect occurring at the junction of two bands. In order to avoid this potential problem. the band was only split in two: above and below $1.5 \mathrm{KHz}$. Figure 5.11 illustrates how the bands are split. A 99 taps lo v-pass McMellan filter is applied to a signal $\mathbf{x}(\mathbf{t})$. In a second branch, the same signal is delayed by 49 taps in order to keep the two branch in phase. The low-pass signal is then coherently subtracted from the delayed original signal which provides the upper band signal.

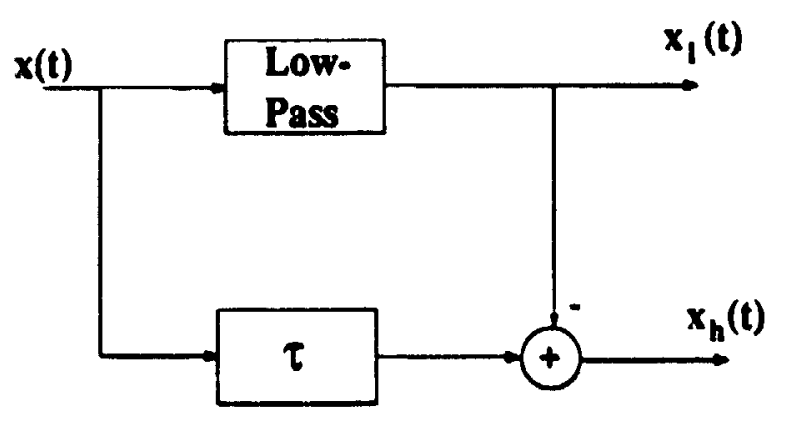

Figure 5.11 Low-Pass and High-Pass Filtering

Figure 5.12 shows the sub-band ANC configuration for lower band adaptation. The primary signal $\mathbf{x}(t)$ is divided in two branches. The first is low-pass fil:sred then a delay $\tau_{2}$ is added to it for ensuring system causality with respect to the reference signal $y(t)$. The second branch is delayed by $\tau_{1}$ in order to compensate for the delay stemming from low-pass filtering and to compensate for the adaptive filter. The signal estimation and optimization are obtained using the lower band. The signal estimation $\hat{\mathbf{x}}(\mathbf{t})$ is also coherently subtracted from the delayed primary signal $x(t)$ [2]. 


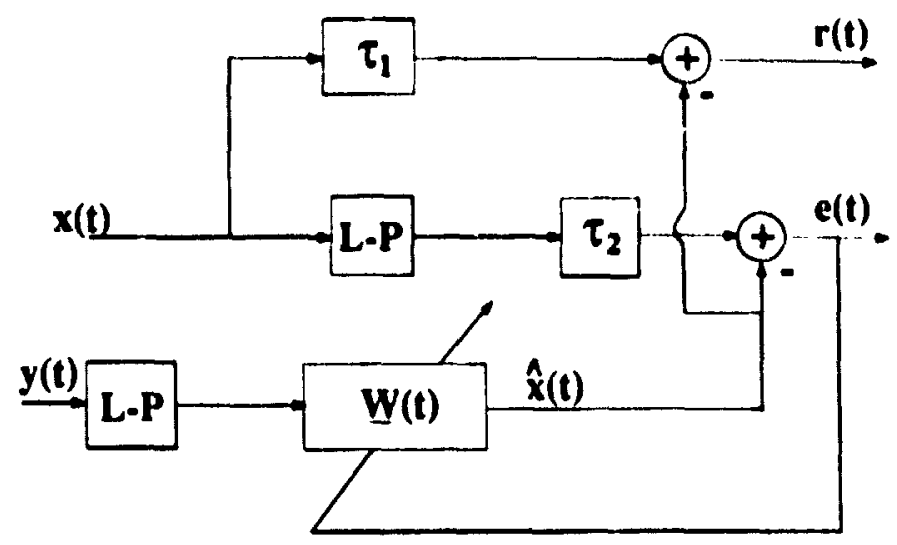

Figure 5.12 Sub-Band Two MicrophoneANC Configuration

\subsection{Results of Sub-Band Adaptation}

The results of sub-band adaptation are presented in three part. First. su: ic reculli s: lower band adaptation are presented. Second, some results of the upper bantu adaptation are presented. Third, sub-band adaptation results of both the upper and lower band are presented. In this case upper and lower band estimation is done independently. It is found that the SNR improvement is about $11 \mathrm{~dB}$. Then, the upper band estimate and the lower band estimate are subtracted from the primary outpui.

\subsubsection{Lower Band Adaptation}

Figures 5.13-5.15 show some of the lower band adaptation. Good noise suppression of the lower band is obtained without causing any effect on the upper band. Further more, every observations made on the lower band of figures 5.4-5.10 still hold. In that case adaptation was performed over the entire acoustic band. Hence, loc tion \# 2 provides the best noise suppression for the frequencies below $800 \mathrm{~Hz}$. Location \#3 performs best for the frequency regions ranging from $800 \mathrm{~Hz}$ to $1500 \mathrm{~Hz}$. 
Mic. \# 1 Under the Hood

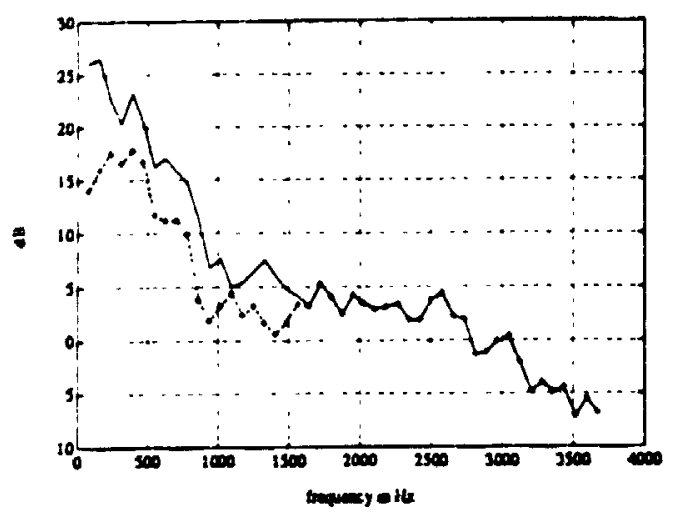

a.
Mic. \# 2 Under the Steering

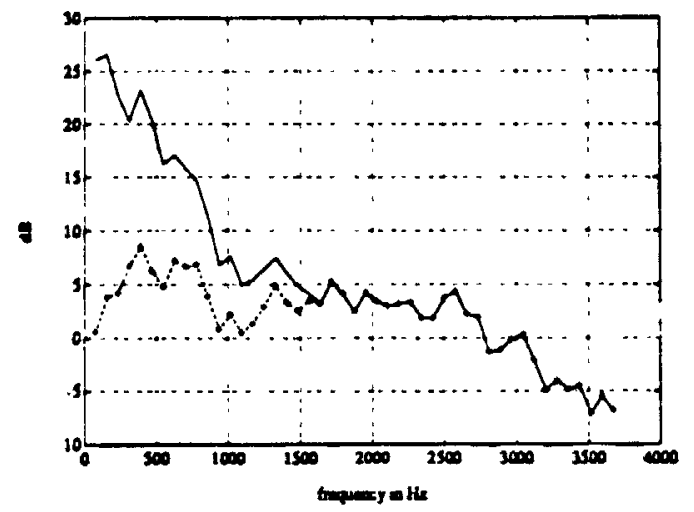

b.

Mic. \# 3 Under Floor Mat

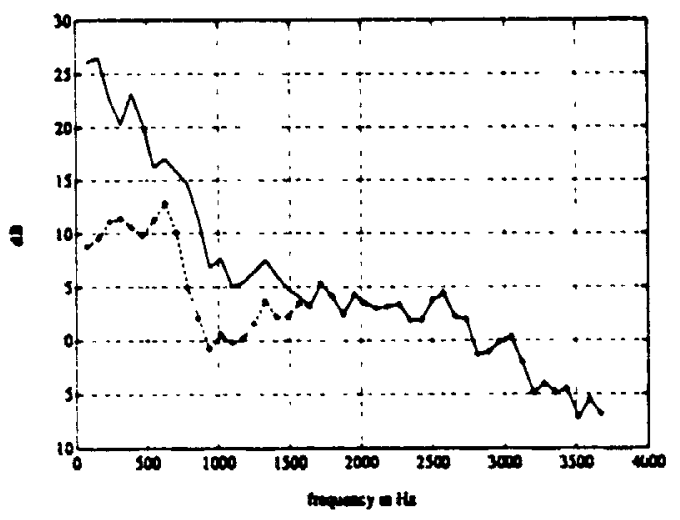

c.

Figure 5.13 Result Of a Two MicrophoneSub-Band ANC (lower band filtering), Car Idling, Fan Off; Output :---

\subsubsection{Upper Band Adaptation}

The results of the upper band adaptation confirms that in addition of uncorrelated 
Mic. \# 1 Under the Hood

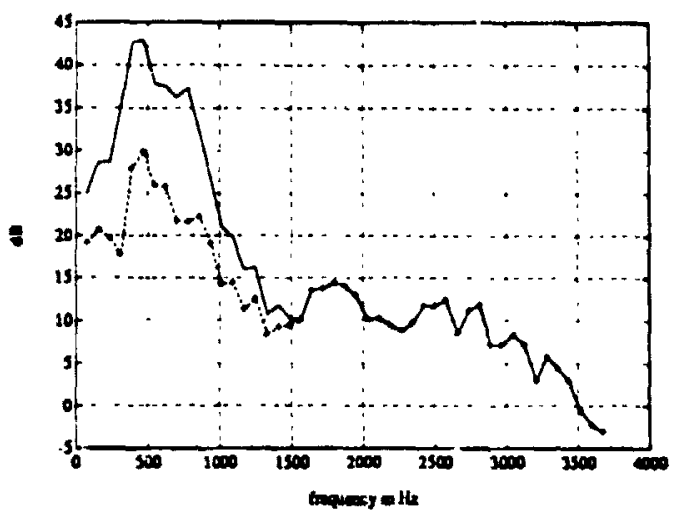

a.
Mic. \# 2 Under the Steering

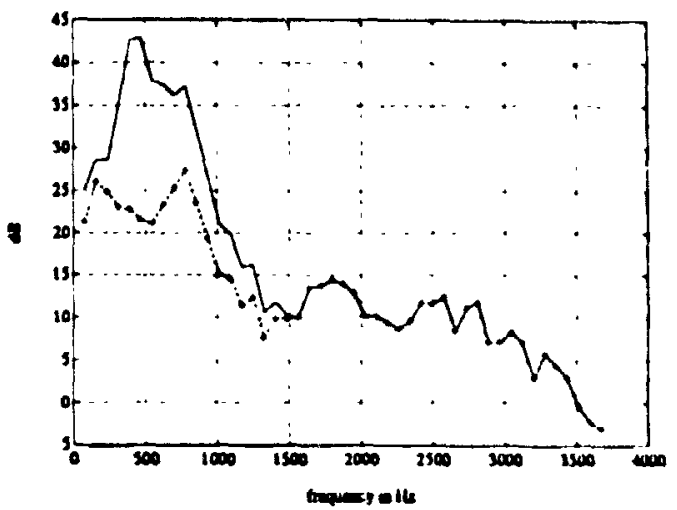

b.

Mic. \# 3 Under Floor Mat

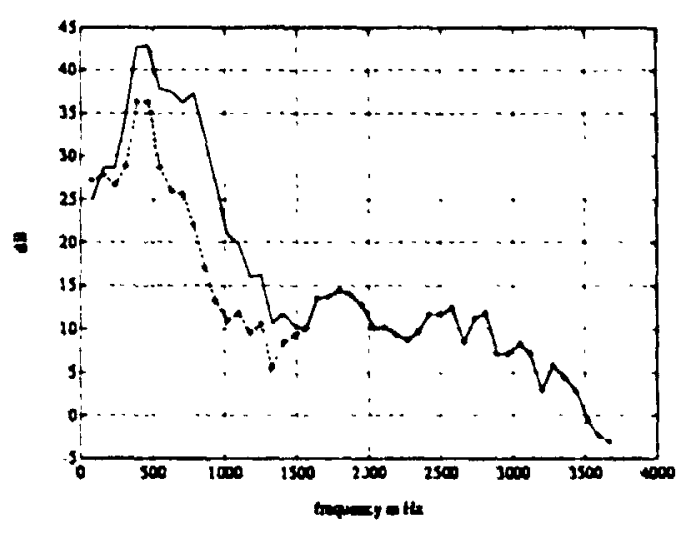

c.

Figure 5.14 Result Of a Two MicrophoneSub-Band ANC (lower band filtering), Car Idling, Fan On; Output :-+-

acoustic noise, there is some correlated noise in the upper band. This was expected after completing the noise analysis. Figure 5.16-5.18 illustrates some of the upper band 
Mic. \# 1 Under the Hood

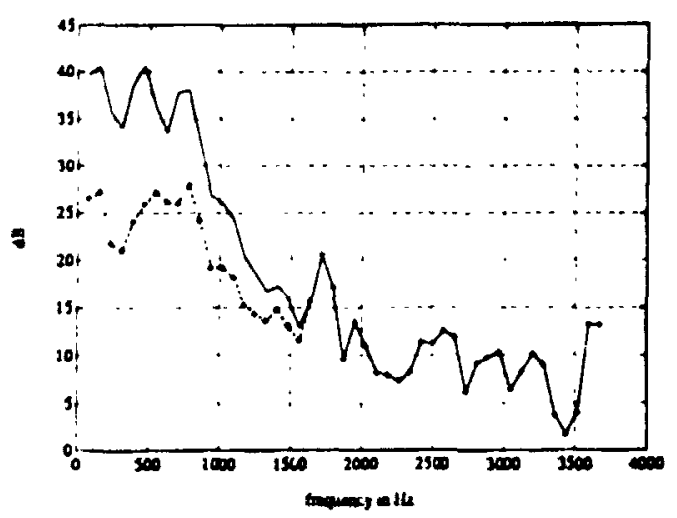

a.
Mic. \# 2 Under the Steering

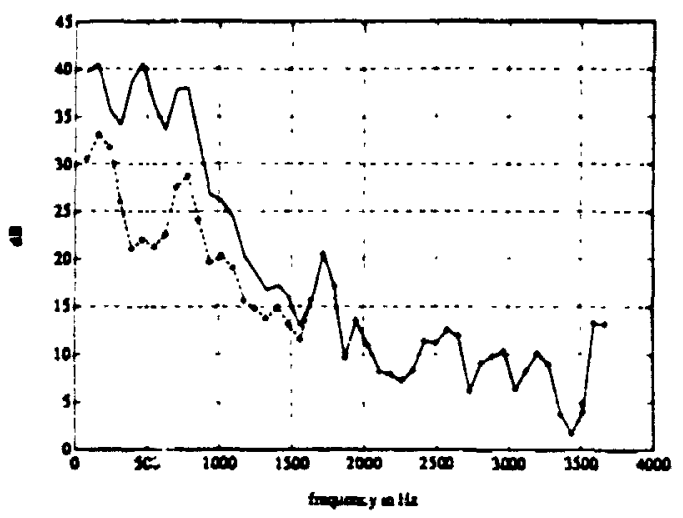

b.

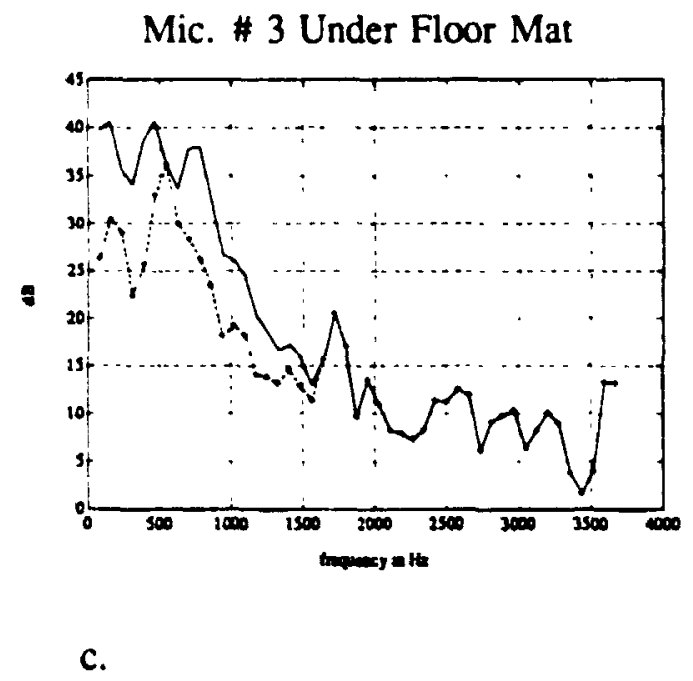

Figure 5.15 Result Of a Two MicruphoneSub-Band ANC (lower band filtering), Car Speed $100 \mathrm{Km} / \mathrm{hr}$, Fan On; Output :-+analysis results.

For all driving conditions, the lower band is not affected by the adaptation. However, 
it is found that the degree of noise suppression varies with the driving conditions and with the considered location.

Figure 5.16 shows the case where the engine is off and the fan is on. Loration \# 1 (under the hood) proved to be the best reference for noise suppression. It was pointed out in chapter 4 that location \# 1 was the most correlated with respect to the primary. Note that there is good noise cancellation at specific frequency regions. However, there is a slight noise enhancement in the frequency region ranging from $2800-3200 \mathrm{~Hz}$.

Figure 5.17 shows the case where the fan is off and the car idles. In this condition, no particular peak could be observed in the upper band. As shown in figure 5.17, for every location, there is noise enhancement. This stresses the fact that only uncorrelated noise is active in the upper band.

Figure 5.18 shows the condition where the car moves at $60 \mathrm{Km} / \mathrm{hr}$ with the fan on. The power peak at $3000 \mathrm{~Hz}$ war found to be common to both the primary (at the steering wheel) and location \# 1 (under the hood). Based on chapter 4 analysis, it was expected to obtain a resulting PSD leveled with the "background noise floor". Instead, a major hole in the PSD results from the noise suppression. This would seem to indicate that some correlated noise are buried in the uncorrelated noise.

\subsubsection{Sub-Band Adaptation Over Both Band}

Figures 5.19-5.25 show the results of sub-band adaptation simultaneously performed on both bands. The general observations are that lucation \# 2 (under the steering wheel) provides good noise suppression for the lower band. It does provide slight noise suppression in the upper band without any noise enhancement. This is only true for the cases where correlated noise can be found in the upper band. 
Mic. \# 1 Under the Hood

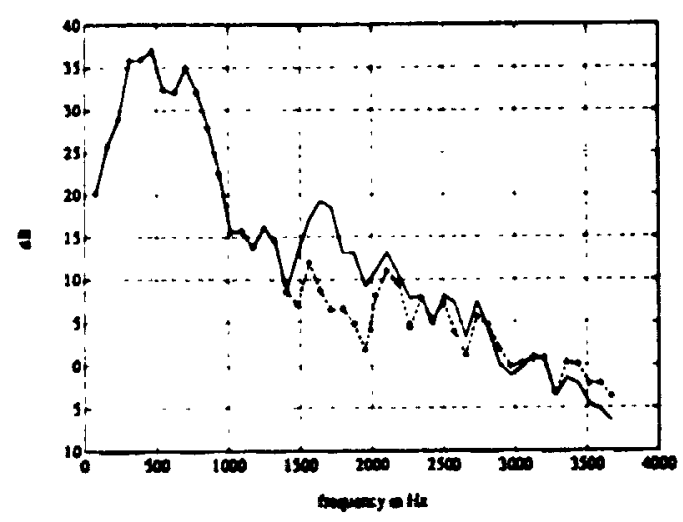

a.
Mic. \# 2 Under the Steering

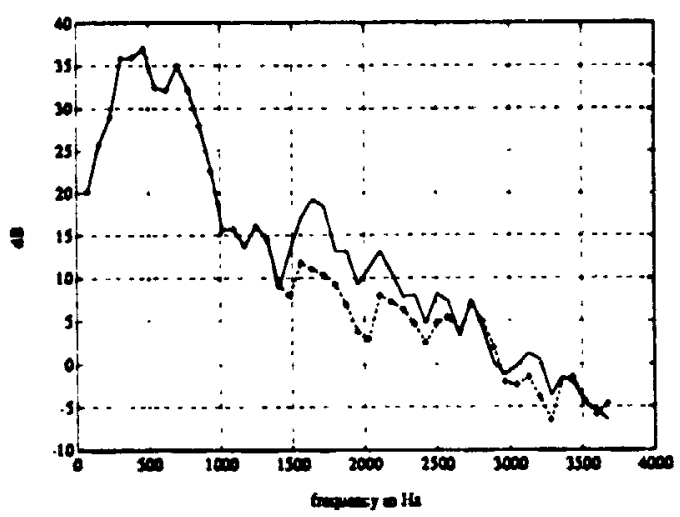

b.

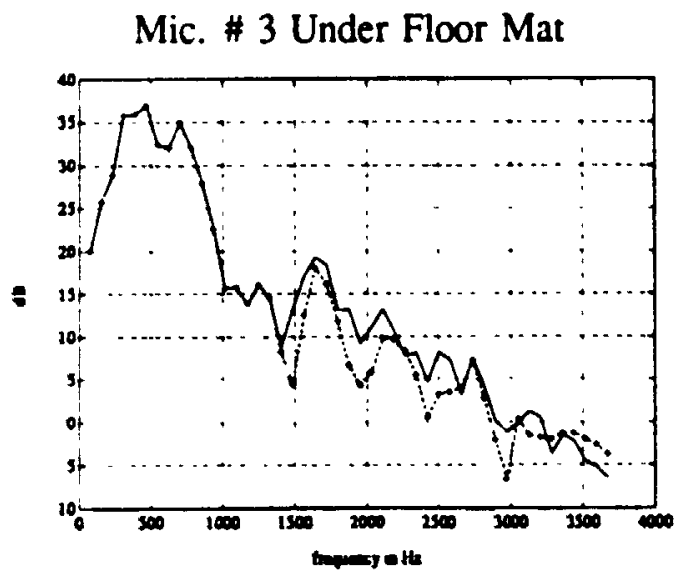

c.

Figure 5.16 Result Of a Two MicrophoneSub-Band ANC (upper band filtering), Engine Off, Fan On; Output :---

Location \# 1 (under the hood) performs fairly well. It provide noise suppression in the lower band as well as in the upper band high end. However, in some conditions, it 
Mic. \# 1 Under the Hood

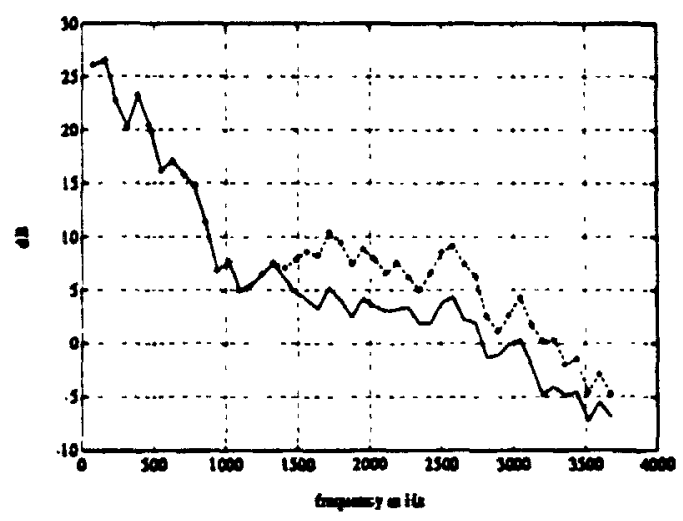

a.
Mic. \# 2 Under the Steering

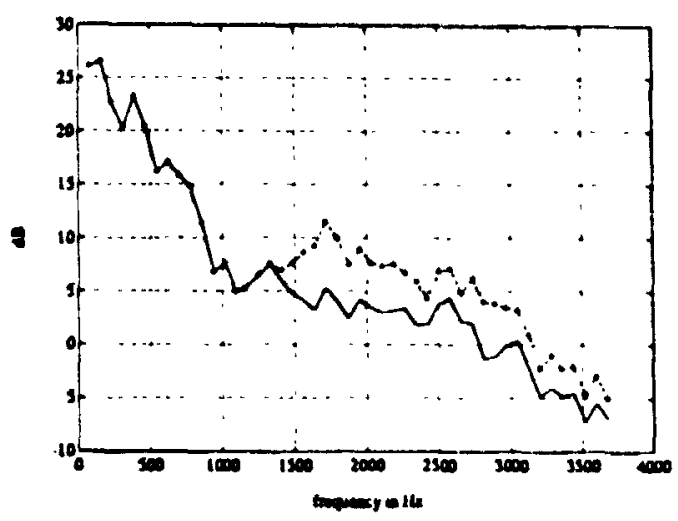

b.

Mic. \# 3 Under Floor Mat

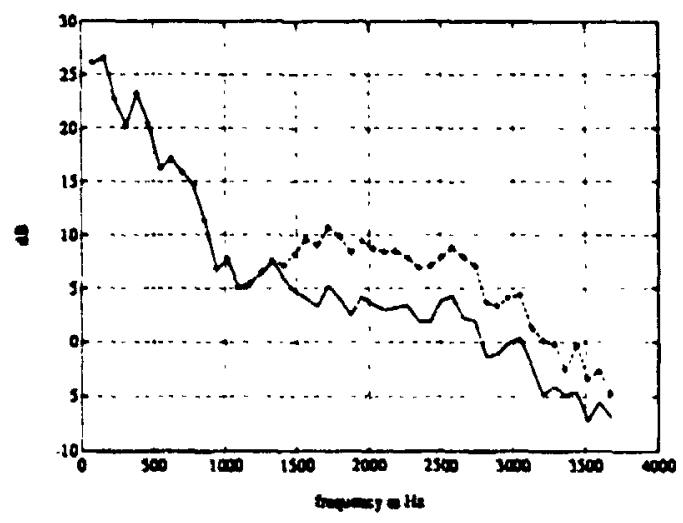

c.

Figure 5.17 Result Of a Two MicrophoneSub-Band ANC (uj'per band filtering), Car Idling, Fan Off; Output :-+provides some noise enhancement in certain part of the upper band spectrum.

Location \# 3 (under the floor mat) performs in a similar way when compared to 
Mic. \# 1 Under the Hood

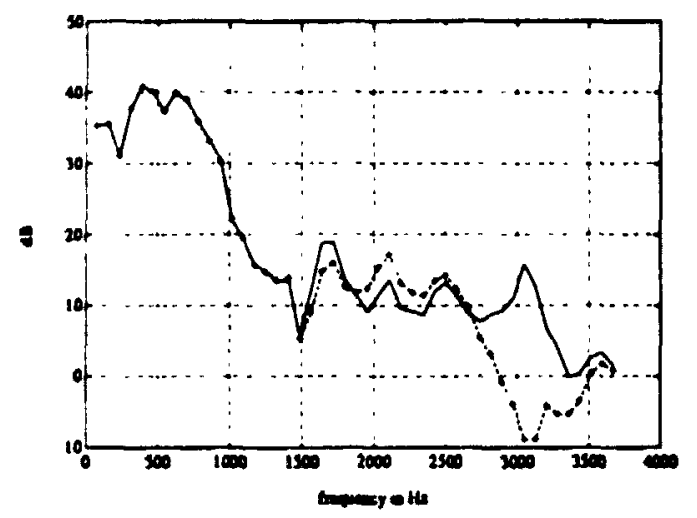

a.
Mic. \# 2 Under the Steering

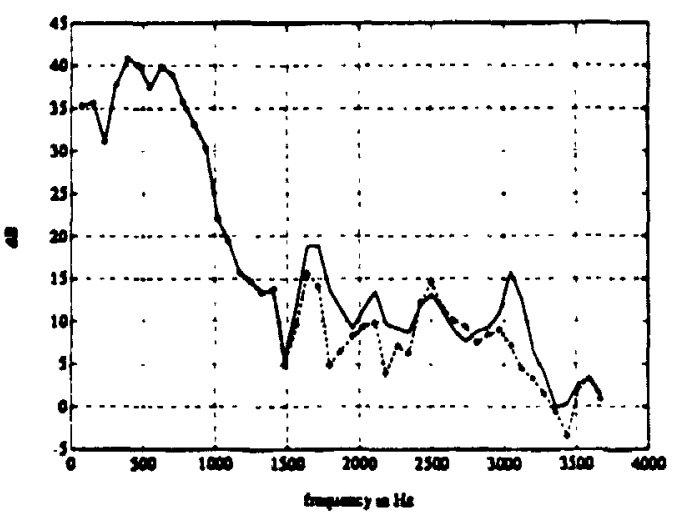

b.

Mic. \# 3 Under Floor Mat

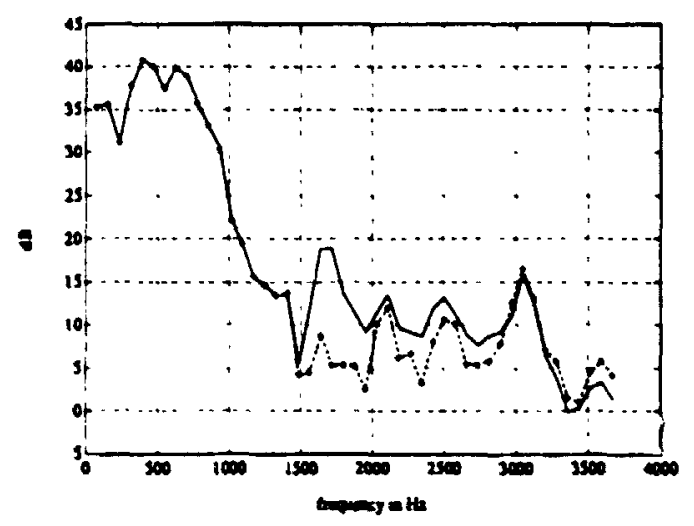

c.

Figure 5.18 Result Of a Two MicrophoneSub-Band ANC (upper band filtering), Car Speed $60 \mathrm{Km} / \mathrm{hr}$, Fan On; Output :---

location \# 1. However, it seems to be effective in the lower end of the upper band.

This section showed that sub-band adaptation solves the problem of noise enhance- 
Mic. \# 1 Under the Hood

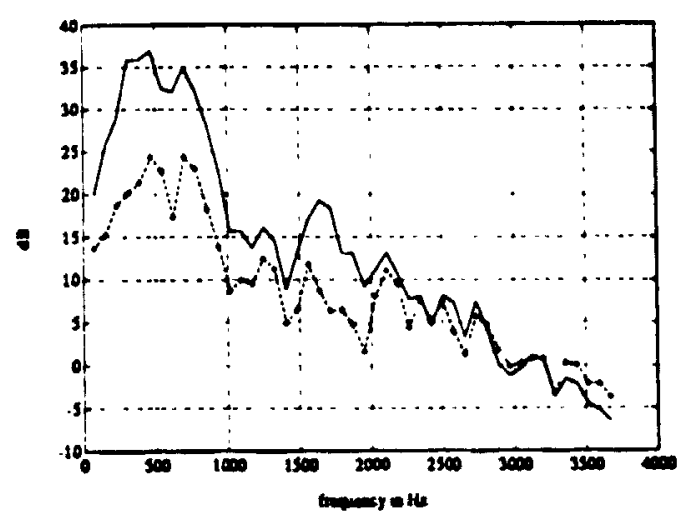

a.
Mic. \# 2 Under the Steering

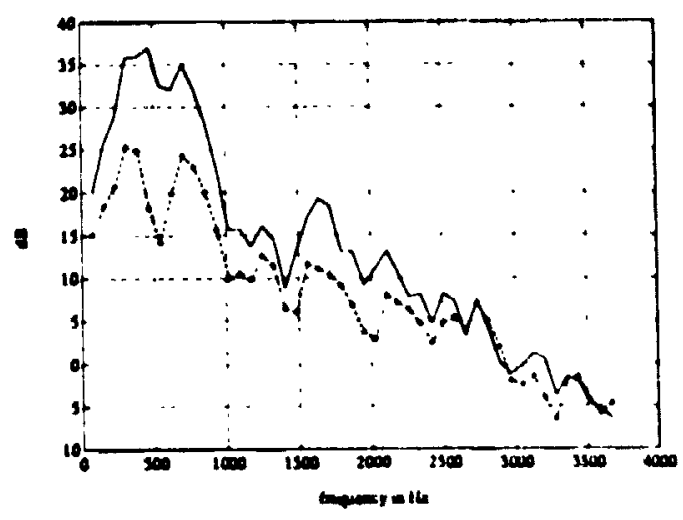

b.

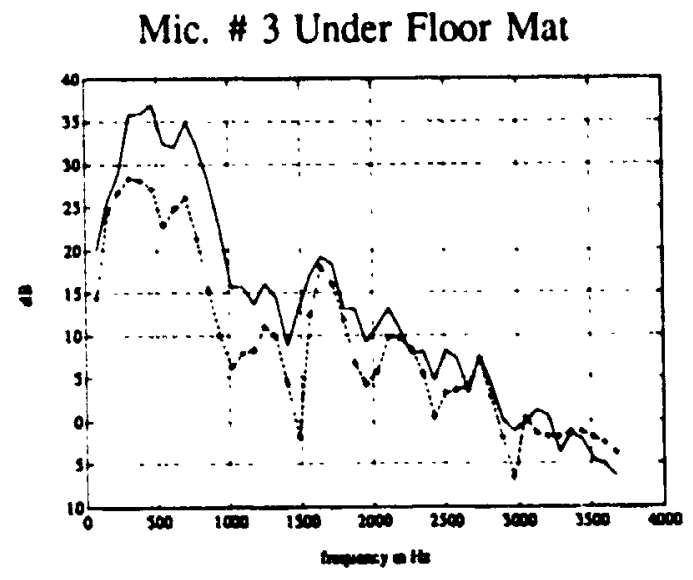

c.

Figure 5.19 Result Of a Two MicrophoneSub-Band ANC (upper and lower band independently filtered), Engine Off, Fan On; Output :---

ment of the upper band. This also confirms that different noise processes are found in the lower and upper bands. 
Mic. \# 1 Under the Hood

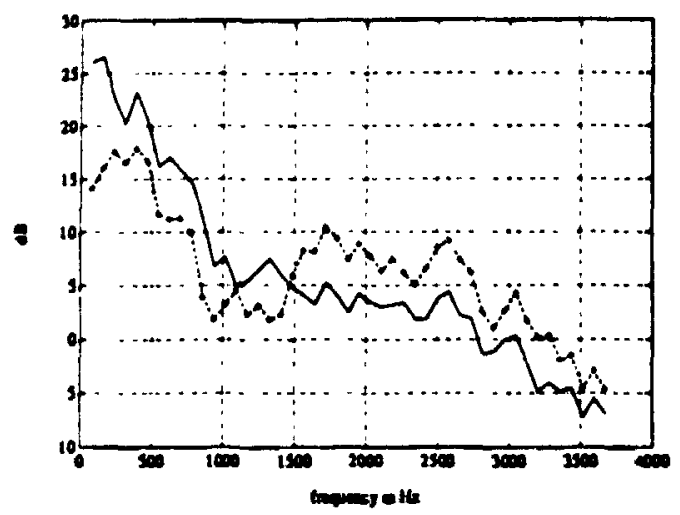

a.
Mic. \# 2 Under the Steering

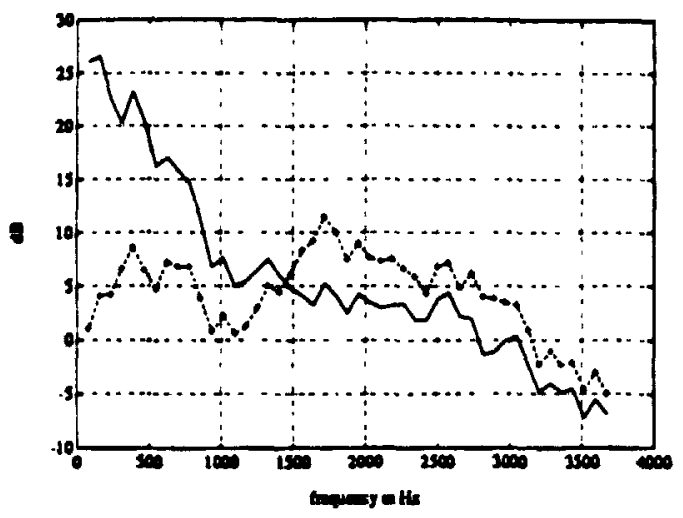

b.

Mic. \# 3 Under Floor Mat

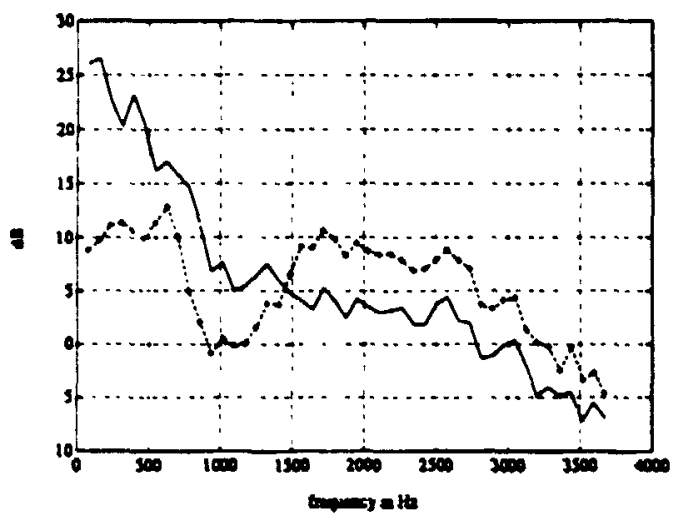

c.

Figure 5.20 Result Of a Two MicrophoneSub-Band ANC (upper and lower band independently filtered), Car Idling, Fan Off; Output :-+- 
Mic. \# 1 Under the Hood

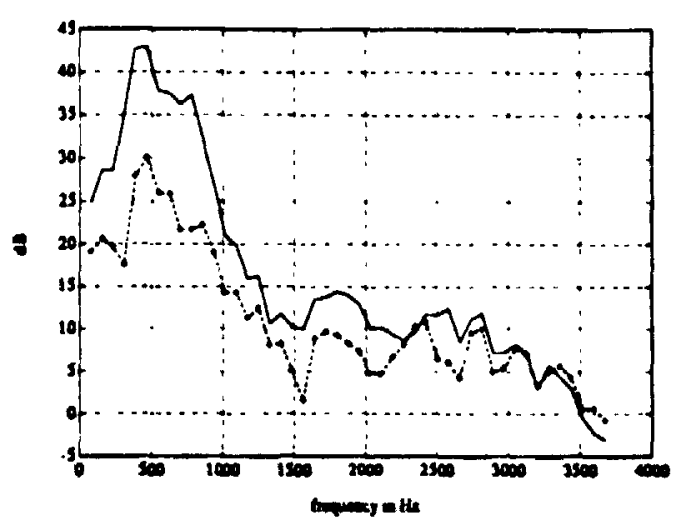

a.
Mic. \# 2 Under the Steering

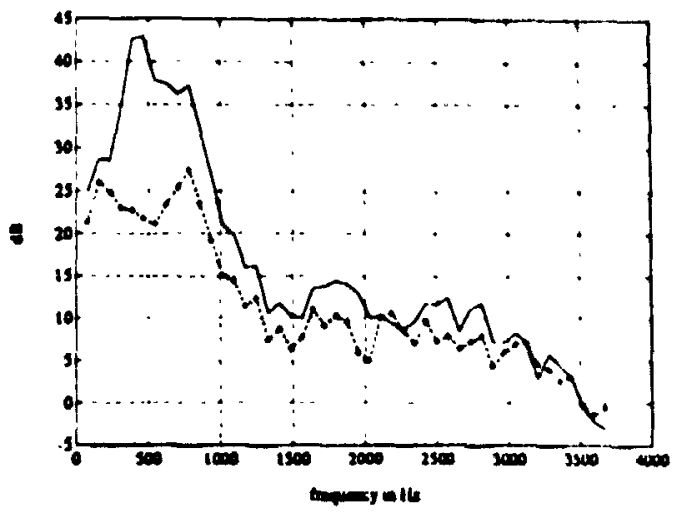

b.

Mic. \# 3 Under Floor Mat

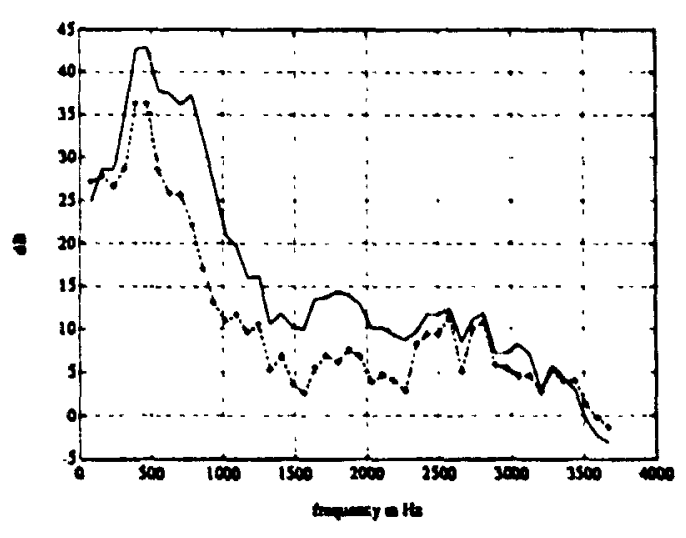

c.

Figure 5.21 Result Of a Two MicrophoneSub-Band ANC (upper and lower band independently filtered), Car Idling, Fan On; Output :-+- 
Mic. \# 1 Under the Hood

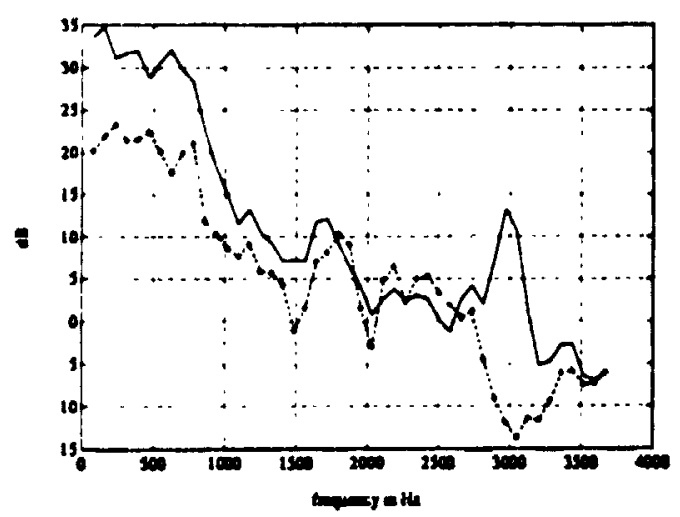

a.
Mic. \# 2 Under the Steering

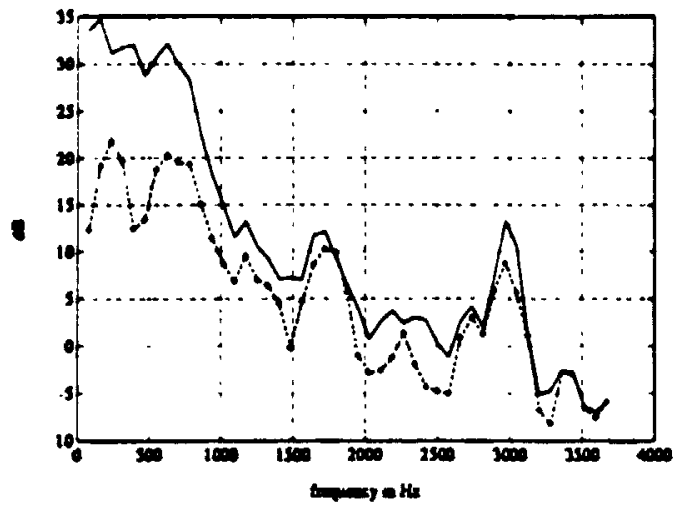

b.

Mic. \# 3 Under Floor Mat

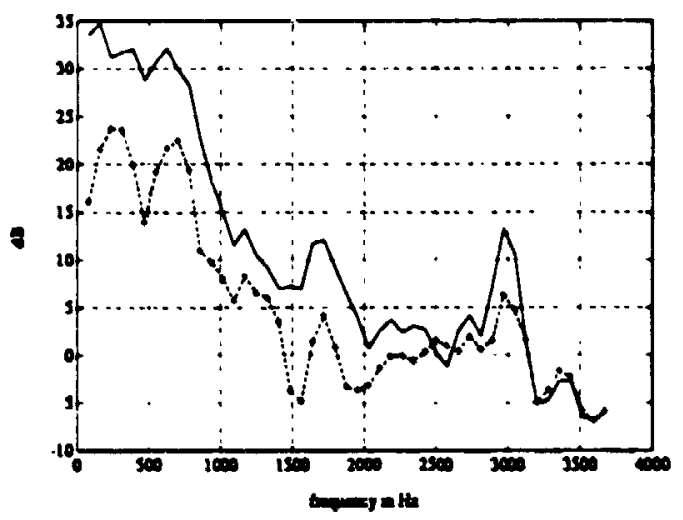

c.

Figure 5.22 Result Of a Two MicrophoneSub-Band ANC (upper and lower band independently filtered), Car Speed $60 \mathrm{Km} / \mathrm{hr}$, Fan Off; Output :-- 
Mic. \#1 Under the Hood

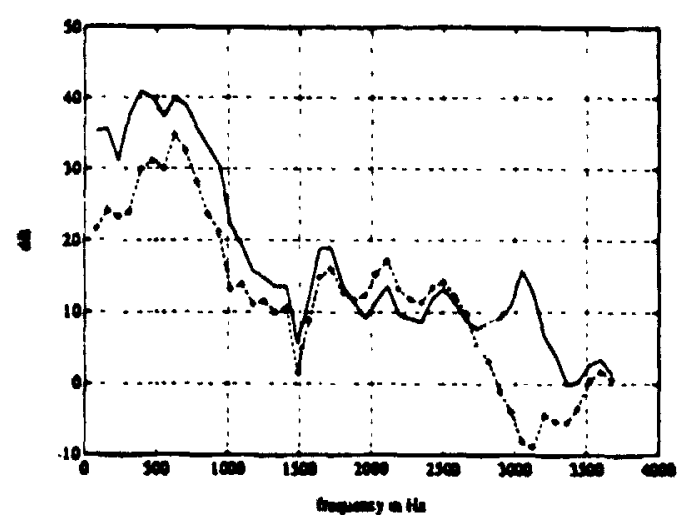

a.
Mic. \# 2 Under the Steering

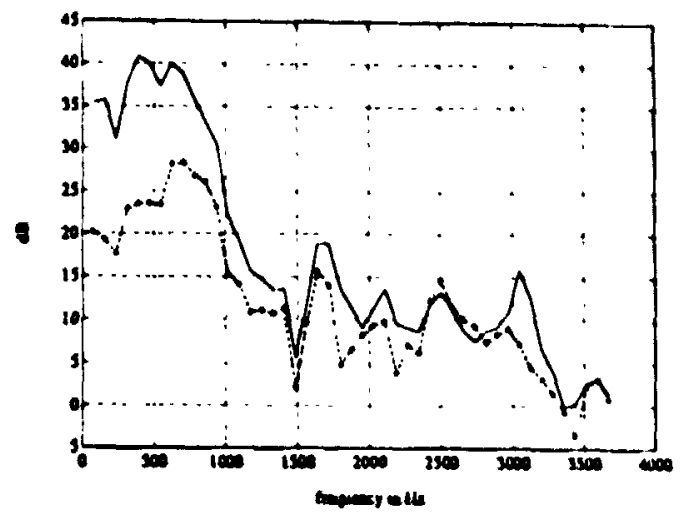

b.

Mic. \# 3 Under Floor Mat

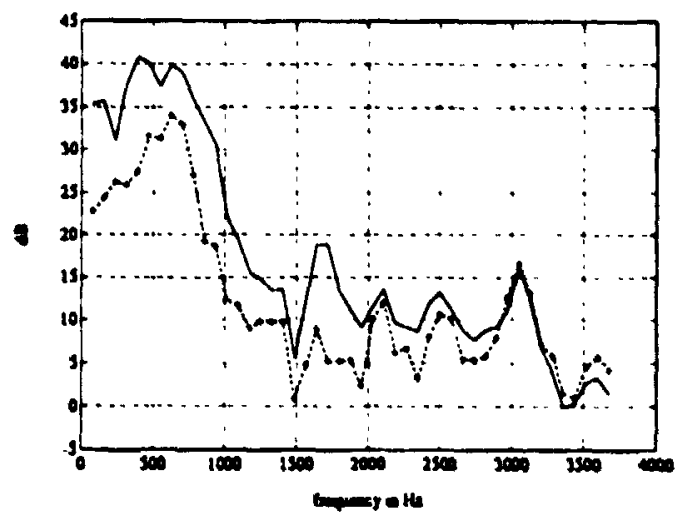

c.

Figure 5.23 Result Of a Two MicrophoneSub-Band ANC (upper and lower band independently filtered), Car Speed $60 \mathrm{Km} / \mathrm{hr}$, Fan On; Output :-+- 
Mic. \# 1 Under the Hood

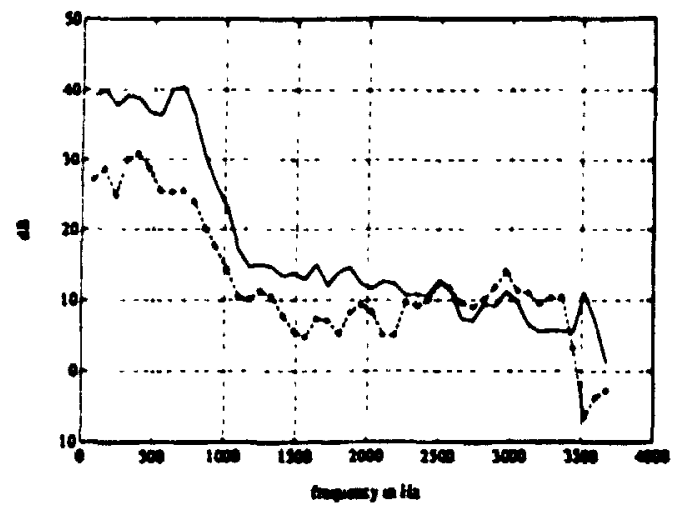

a.
Mic. * 2 Under the Steering

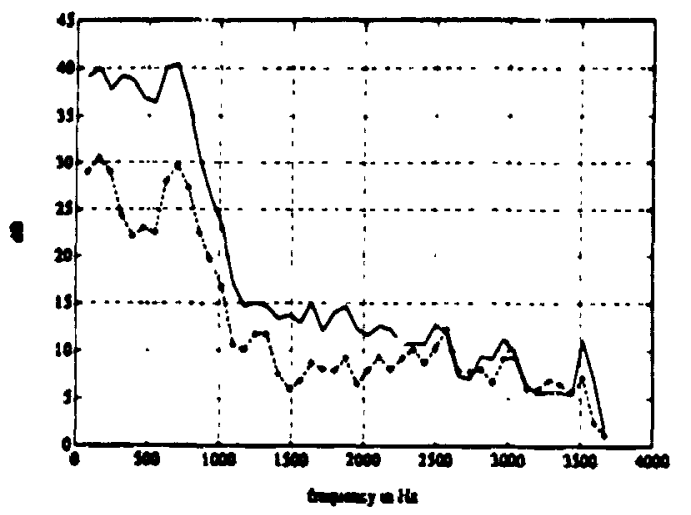

b.

Mic. \# 3 Under Floor Mat

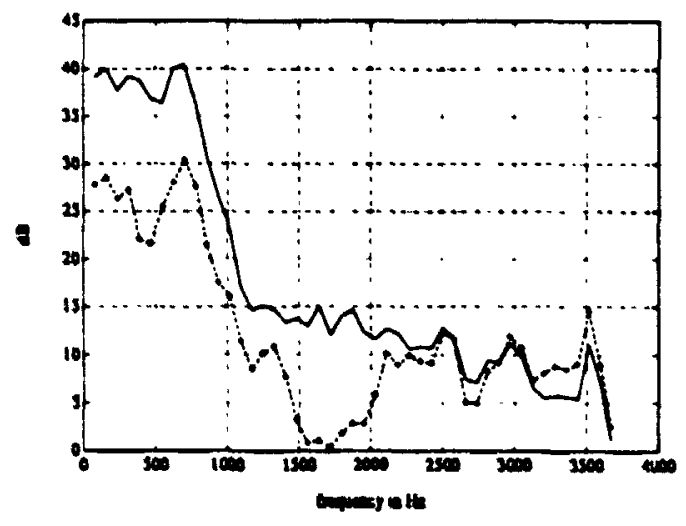

c.

Figure 5.24 Result Of a Two MicrophoneSub-Band ANC (upper and lower band independently filtered), Car Speed $100 \mathrm{Kr}$.arr, Fan Off; Output :-+ 
Mic. \# 1 Under the Hood

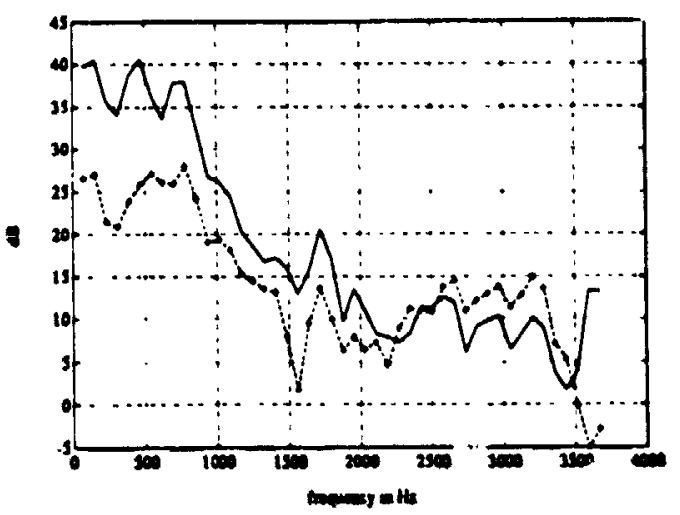

a.
Mic. 2 Under the Steering

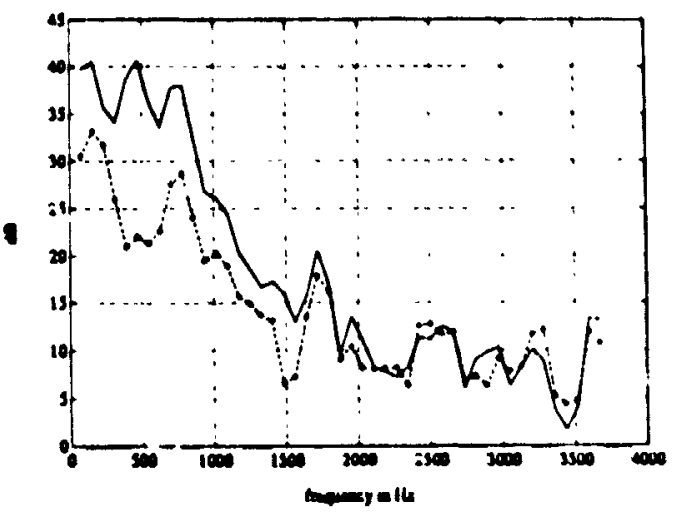

b.

Mic. \# 3 Under Floor Mat

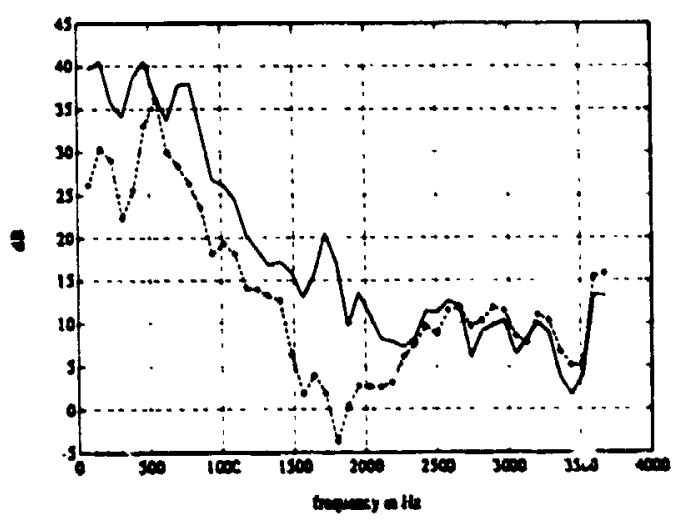

c.

Figure 5.25 Result Of a Two MicrophoneSub-Banci ANC (upper and lower band independently filtered), Car Speed $100 \mathrm{Km} / \mathrm{hr}$, Fan On; Output: -+- 

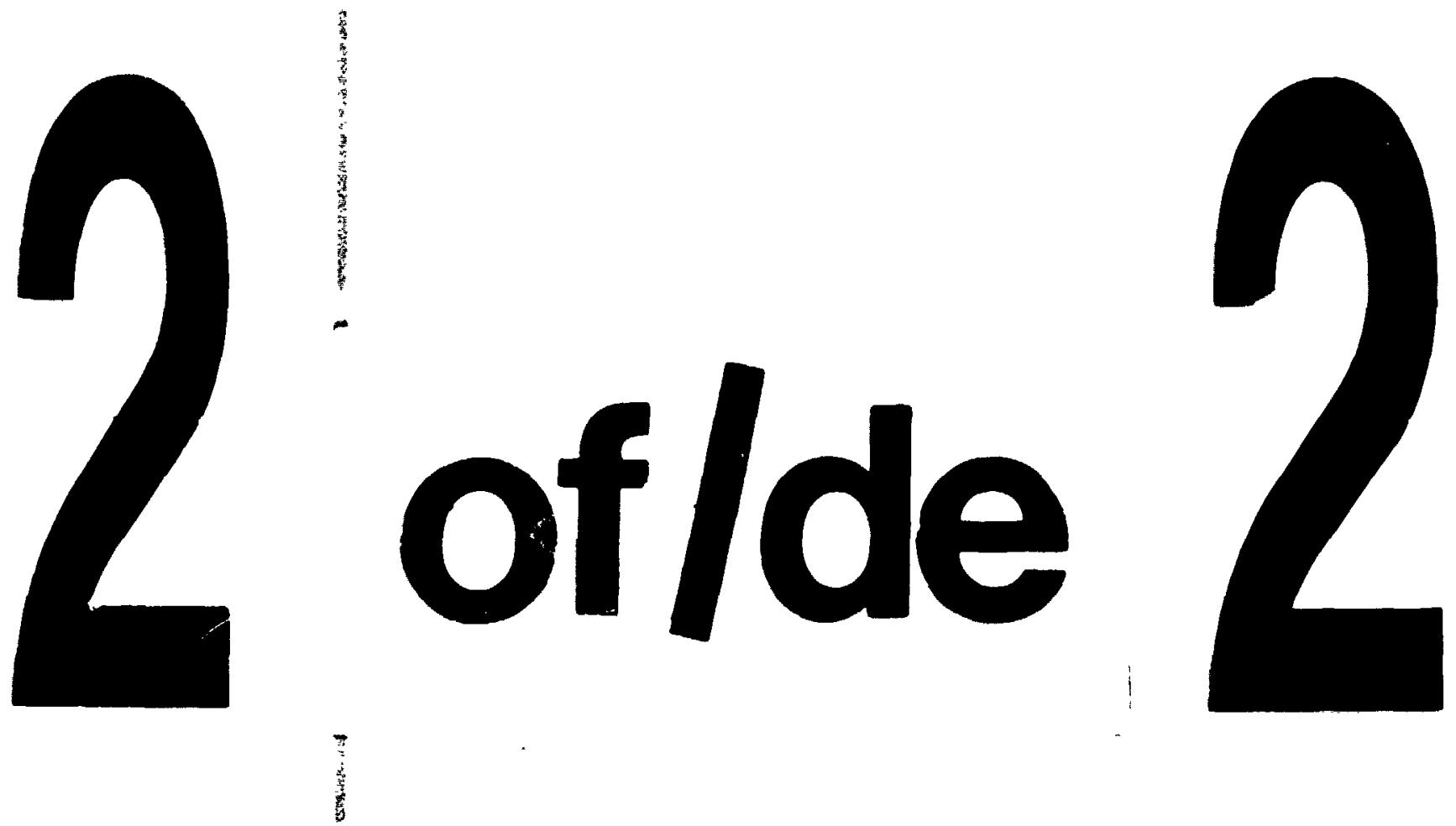

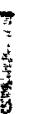

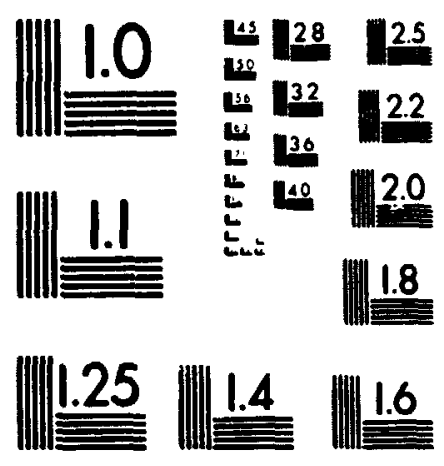

MICROCOPY RESOLUTION TEST CHART

NATIONAL GUREAU OF STANOARDS

STANDARD REFEAENCE MATERIAL 1010

(ANSI and ISO TEST CMART NO. 2)

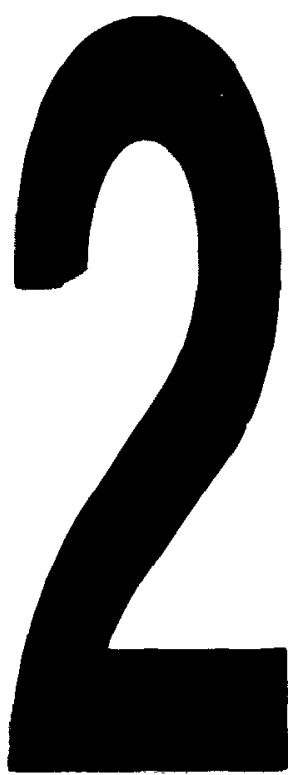




\section{Chapter 6 Parallel Adaptation}

\subsection{Introduction}

It was shown in chapter 5 that some microphones (or locations) perform better than others depending on the driving conditions. This is also true for particular frequency regions as demonstrated in sub-band adaptation. These phenomena were somewhat expected based on the noise analysis in chapter 4 . In light of these phenomena, it would be desirable to develop a multiple reference ANC system which would always make use of the best location and the best frequency contribution. The system could use a parallel architecture similar to that of a beamformer (BF) $[14,19]$. The literature refers to the best location selection as spatial filtering. Further to it, the system should be able to extract from each location the pertinent feature or signal component for achieving a high degree of noise cancellation. For instance, it was shown that some location allows good noise suppression at a particular frequency region. Hence its contrioution should be limited to that frequency region. This frequency region selection is referred to as temporal filtering. In this chapter, two basic configurations based on a BF like approach are presented as well as their results on real car data.

\subsection{General Beamformer Approach}

The introduction of a parallel structure as a back bone for a multiple reference ANC requires a three dimensional analysis i.e. the system output depends on temporal 
and spatial filtering. Temporal for the noise located at a particular frequency region and spatial filtering for selecting the best location for background acoustic noise suppression. Temporal and spatial filtering must be performed simultaneously which renders the system very complex. Figure 6.1 illustrates the concept of a multiple reference ANC.

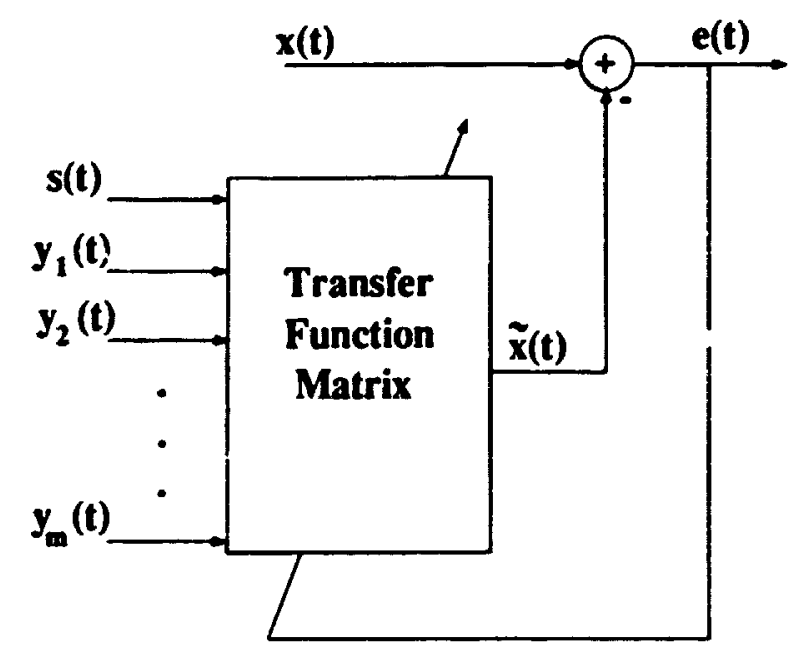

Figure 6.1 Multiple Reference ANC Configuration

A transfer function matrix must be evaluated in order to perform noise cancellation. Using the beamformer approach, the analysis is broken down into two 2-dimensional systems. Each system is analyzed independently. Hence, the structure analysis is broken down into a temporal and spatial part. For ease of analysis, it is assumed that adaptation takes place during speechless period.

\subsubsection{Temporal Filtering}

An algorithm for temporal filtering can be developed in exactly the same way as a 
2 Microphone ANC. Only one reference microphone is considered with respect to all noise sources. This algorithm development was carried through in chapter 5 using the LMS algorithm. Applying, equation 5.27 to the $\mathrm{i}^{\text {th }}$ reference location gives

$$
\underline{\mathbf{w}}_{i}(t+1)=\underline{\mathbf{w}}_{i}(t)+\beta(t) e(t) \underline{\mathbf{y}}_{i}(t)
$$

where $\underline{w}_{1}(t+1):$ is the new set of coefficients;

$\underline{w}_{1}(t):$ is the old set of coefficients;

$\beta(t)$ is the step size normalized to the instantaneous power contained in the sequence $\mathbf{y}_{1}(\mathbf{t})$.

$$
\beta(\mathbf{t})=\frac{\mathbf{a}_{\mathbf{i}}}{\mathbf{b}_{\mathbf{i}}+\underline{\mathbf{y}}^{\mathbf{T}}(\mathbf{t}) \underline{\mathbf{y}_{\mathbf{i}}(\mathbf{t})}}
$$

where $a_{\mathbf{l}}$ and $b_{\mathbf{l}}$ are some limiting constants ensuring system stability when $\mathbf{y}_{\mathbf{I}}(t)$ is close to zero.

$e(t):$ is the difference between the actual primary output $x(t)$ and its estimate $\tilde{\mathbf{x}}(t)$. In the subsequent section a cornplete explanation on the error provenance will be defined in terms of the entire system.

$y_{1}(t)$ : is a $N \times 1$ vector composed of the last $N$ observations of the $i^{\text {th }}$ reference location.

This ends the temporal filtering portion of the analysis. Figure 6.2 illustrated how temporal filtering fits in the entire system.

\subsubsection{Spatial Filtering}

For spatial filtering the system can be represented by figure 6.3. Here the temporal dimension is omitted. This simplifies the spatial filtering analysis. 

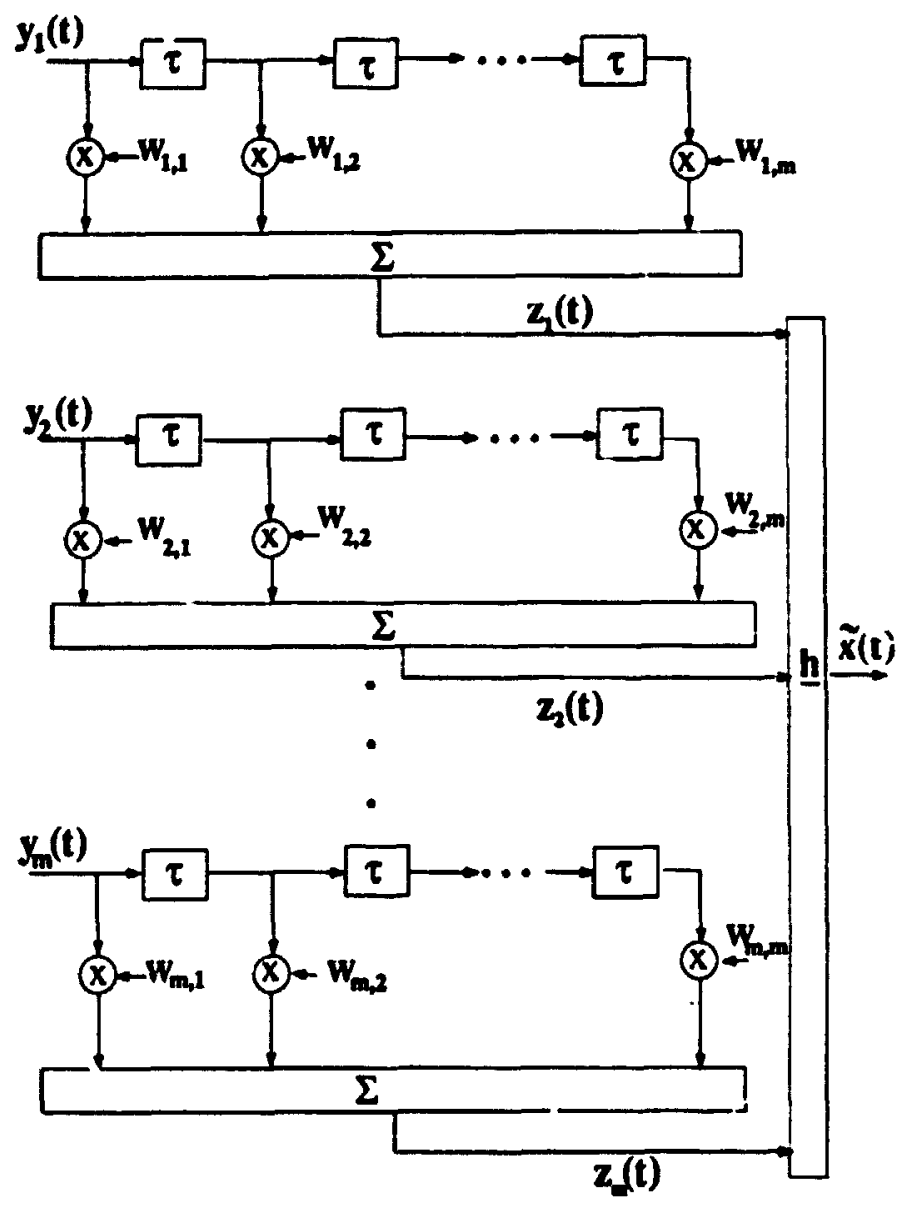

Figure 6.2 Tempora, Filtering in the Multiple Reference ANC

Spatial filtering consists in estimating the primary output $x(t)$ by a linear combination of the filtered reference output (temporally filtered) $\underline{z}(t)$. The linear combination is then 


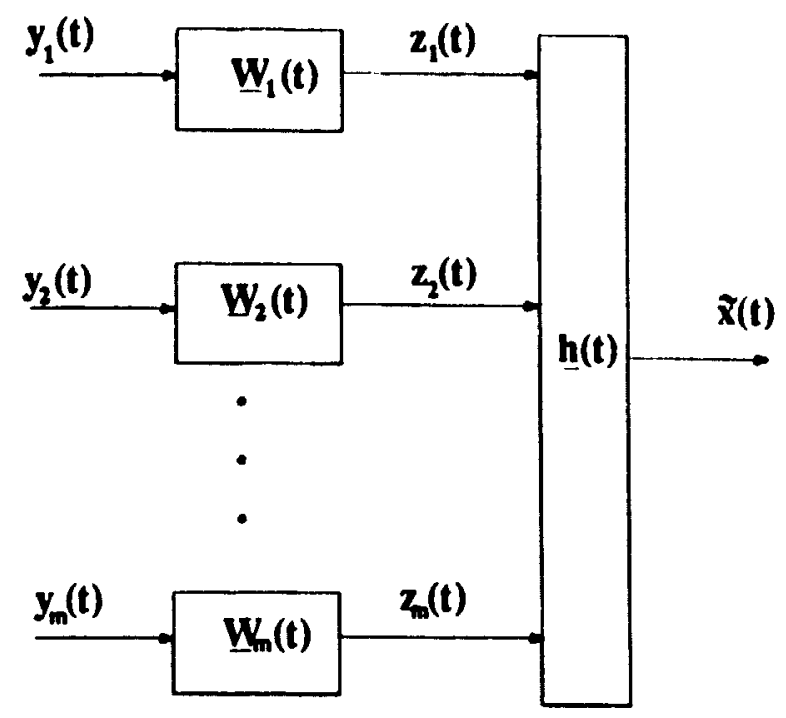

Figure 6.3 Spatial Filtering Configuration

minimized in a mean square sense.

$$
\underline{z}(c)=\left[\begin{array}{c}
z_{1}(t) \\
z_{2}(t) \\
\cdot \\
\cdot \\
\cdot \\
z_{m}(t)
\end{array}\right]
$$

where

$$
\mathbf{z}_{\mathbf{i}}(\mathbf{t})={\underline{y_{i}}}_{\mathbf{T}}^{\mathbf{T}}(\mathbf{t}) \underline{\mathbf{W}}_{\mathbf{i}}(t)
$$

the estimation error is given by the difference between the actual primary output $x(t)$ and its estimate $\tilde{\mathbf{x}}(\mathbf{t})$

$$
e(t)=x(t)-\tilde{x}(t)
$$

where

$$
\overline{\mathbf{x}}(\mathbf{t})=\underline{\mathbf{z}}^{\mathbf{T}}(\mathbf{t}) \underline{\mathbf{h}}(\mathbf{t})
$$


As previously obtained in chapter 5 , the mse is

$$
\text { mse }=E\left[e^{2}(t)\right]=E\left[x^{2}(t)-2 x(t) \tilde{x}(t)+\bar{x}^{2}(t)\right]
$$

substituting equation (6.6) in (6.7) and assuming that the system statistics are constant over the period of observation, the mse becomes

$$
\begin{aligned}
\text { mse }=E\left[x^{2}(t)\right]- & 2 \underline{h}^{T} E[\underline{z}(t) x(t)]+\underline{h}^{T}(t) E\left[\underline{z}(t) \underline{z}^{T}(t)\right] \underline{h}(t) \\
& =\sigma_{x}^{2}-2 \underline{h}^{T}(t) \underline{p}+\underline{h}^{T} R_{z} \underline{h}
\end{aligned}
$$

where $\mathbf{p}$ is the cross-correlation between the primary output $\mathbf{x}(t)$ and the filtered references output $\mathbf{z}(\mathbf{t})$

$$
\underline{p}=\mathbf{E}[\mathbf{x}(\mathbf{t}) \underline{\mathbf{z}}(\mathbf{t})]
$$

$\mathbf{R}_{\mathbf{z}}$ is the autocorrelation of the sequence $\mathbf{z}(\mathbf{t})$

$$
\mathbf{R}_{\mathbf{z}}=\mathbf{E}\left[\underline{\mathbf{z}}(\mathbf{t}) \underline{\mathbf{z}}^{\mathbf{T}}(\mathbf{t})\right]
$$

For ease of manipulation, it is assumed that each reference output are independent of one another with zero mean. The $i^{\text {th }}$ filtered output reference has a variance $\sigma_{\frac{\pi}{4}}^{2}$. Substinuting equation (6.3) in (6.11), the autocor__lation becomes

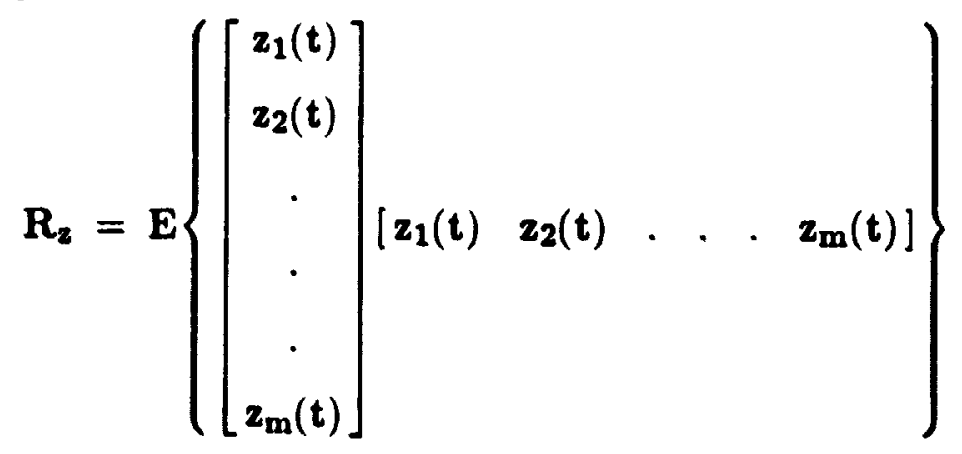




$$
=\left[\begin{array}{cccccc}
\sigma_{\mathrm{z}_{1}}^{2} & 0 & & & 0 \\
0 & \sigma_{\mathrm{z}_{2}}^{2} & & & & \cdot \\
& & \cdot & & \cdot \\
& & & \cdot & & \cdot \\
& & & & \cdot & 0 \\
0 & . & . & \cdot & 0 & \sigma_{z_{w}}^{2}
\end{array}\right]
$$

By inspection of equation (6.7), the mse is strictly positive. It has the shape of a cup facing upward when plotted against the filter coefficient $\underline{h}(\mathbf{t})$. The bottom of the cup coincides with the minimum mse. It is obtained by a set of optimum coefficient hopt. The optimum set of coefficient is obtained by taking the partial derivative of equation (6.9) with respect to $\underline{h}$ and to setting to zero.

$$
\frac{\partial \mathrm{mse}}{\partial \underline{\mathbf{h}}}=-2 \underline{\mathbf{p}}+2 \mathbf{R}_{\mathbf{2}} \underline{\mathbf{h}}_{\text {opt }}=0
$$

the optimum set of coefficient is then given by

$$
\underline{\mathbf{h}}_{\mathrm{opt}}=\mathbf{R}_{\mathbf{z}}^{-1} \underline{\mathbf{p}}
$$

Again, this is the well known Wiener-Hopf equation. This concludes the spatial filtering analysis. Now, the $\boldsymbol{n}$ is to integrate both temporal and spatial filtering in an algorithm using multiple references. Prior to doing so, some constraints may be defined and forced onto the system in order to optimize its use.

\subsection{Use Of Constraint For Algorithm Optimization}

A multiple zeference ANC is a very complex system. As shown in the previous section, two levels of adaptation must be performed: temporal and spatial. In order to 
alleviate the system task and to ensure system stability, sorne constraints may be defined and forced onto it.

The first constraint results trom the work done in chap:sr 5. It was shown that adaptation over the entire acoustic band provides good noise suppression in the lower band while yielding noise enhancement in the upper band. Therefore sub-band adaptation is selected as the first constraint.

The second constraint deals with system stability. Through preliminary testing, a two level adaptation multiple reference ANC was implemented. It will be thoroughly described in a subsequent section. The first adaptation level deals with temporal filtering while the second deals with spatial filtering $[2,691$. It was found that the set of coefficients $\underline{h}(t)$ while minimizing the squared error, would grow without bound. Due to the computer's finite arithmetic limitation, the system would always become unstable. The solution lied in the BF approach. The spatial filtered output must be bounded to the primary output level $\mathbf{x}(t)$. This is achieved by normalizing the spatial filter. Using the Lagrange multipliers [14,19], normalization with respect to the primary output is obtained by simply forcing $\underline{h}(t)$ to have a unity norm. Hence

$$
|\mathbf{h}(\mathbf{t})|=\mathbf{1}
$$

\subsection{Microphone Adaptive Noise Cancellation and Results}

In this section, two 4-micrcphone ANC structures arc presented as well as some experimental results. The first structure is a 2 level adaptation system. Temporal and spatial filtering each form a level. The second structure simultaneously tackles both temporal and spatial filtering using a sequential form. 


\subsubsection{Level Adaptation System and Results}

As mentioned earlier, the 2 level adaptation system structure performs (w') tasks. First, temporal filtering is done, spatial filtering follows. This is done in a quasi independent way. The LMS algorithm is implemented for both levels. Figure 6.4 shows the system configuration.

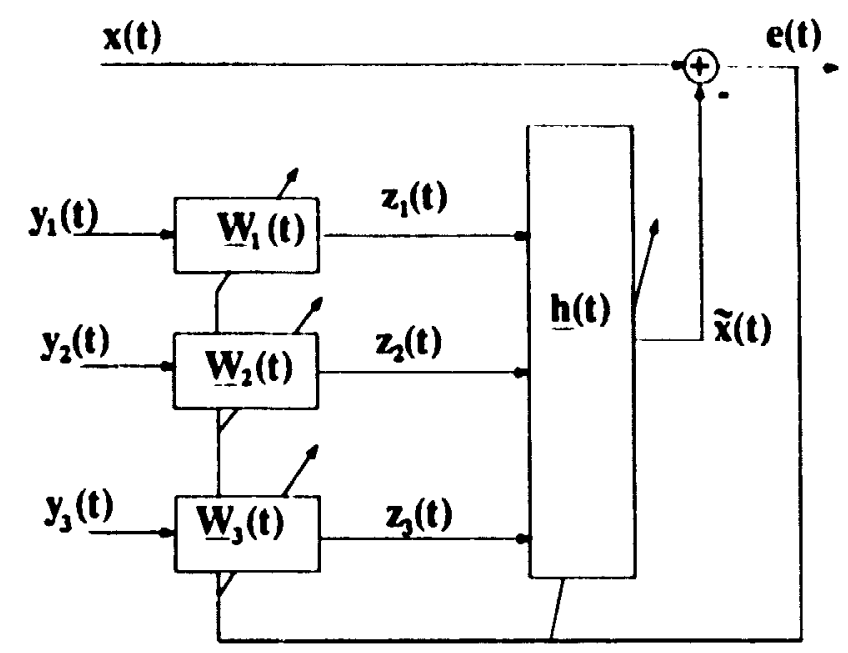

Figure 6.4 Parallel Structure, 2 Level ANC Configuration

At the first level, temporal filtering is performed for each reference microphone. This structure gears or controls each reference microphone contribution with respect to its power level output. I.E. the step size for the $\mathrm{i}^{\text {th }}$ reference microphone $\beta_{i}(t)$ is given by

$$
\beta_{i}(t)=\frac{a_{i}}{b_{i}+\underline{y}_{i}^{T}(t) \underline{y}_{i}(t)}
$$

where $a_{1}$ and $b_{1}$ are some limiting constants bounding the value of $\beta_{1}(t)$. $y_{1}(t)$ is a $N \times 1$ vector composed of the last $\mathrm{N}$ observations from the $\mathrm{i}^{\text {th }}$ reference location. It is known that the power level varies for each location. Evaluating $\beta_{l}(t)$ this way offers a great flexibility since it tends to normalize the primary estimate with respect to the 
power level of each reference. This renders each reference power level transparent to the spatial filter. The system is maximized using a global (or common) error $e(t)$. This error is used for each of the three temporal filters and for the spatial filter. From these definition, equation (5.27) is adapted to the system. Hence each temporal filter set of coefficient are updated using

$$
\underline{W}_{i}(t+1)=\underline{W}_{i}(t)+\beta_{i}(t) e(t) \underline{y}_{i}(t)
$$

where $W_{1}(t+1)$ and $W_{1}(t)$ are respectively the new and old coefficients.

The second level of adaptation performs spatial filtering. The evaluation of the coefficient $\underline{h}(t)$ is crincal in this phase. It was found through simulation and experimentation that $\underline{h}(t)$ must be normalized to unity in order to ensure system stability. Again, using equation (5.27), spatial filtering is ruled by

$$
\underline{h}(t+1)=\underline{h}(t)+\alpha(t) e(t) \underline{z}(t)
$$

subject to

$$
|\underline{h}(t)|=1
$$

this is achieved using

$$
\underline{\mathbf{h}}(\mathbf{t})=\frac{\underline{\mathbf{h}}(\mathbf{t})}{\sum_{\mathbf{i}=1}^{3} h_{i}(t)}
$$

Obviously, $\underline{h}(t+1)$ and $\underline{h}(t)$ are respectively the new and the old coefficients. $\alpha(t)$ is the adaptation step size. This is the most critical factor of the entire systern.

$$
\alpha(t)=\frac{a}{b+\underline{z}^{T}(t) \underline{z}(t)}
$$


$a$ and $b$ are some limiting constant bounding $\alpha(t)$. The variable $z(t)$ is the temporal filter output vector

$$
\underline{z}(t)=\left[\begin{array}{l}
z_{1}(t) \\
z_{2}(t) \\
z_{3}(t)
\end{array}\right]
$$

the error $e(t)$ is the global system error: it is the difference between the primary output and its estimate.

It must be pointed out why evaluating $\alpha(t)$ is so critical. The step size must be such that it tracks the system statistics variation without reacting to the temporal estimation errors. Through experimental fine tuning, it is found that the constant a must be set 0.5 as a maximum otherwise the system is unstable. Despite this, the structure reveals itself to sometimes lose its tracking capability. An in depth investigation should be carried out on the step size selection effect on the system tracking capability.

The 2 level adaptation structure is very effective in suppressing the lower band acoustic noise. This is shown in figures 6.5 and 6.6 .

Figure 6.5 shows a noise suppression of up to $20 \mathrm{dBs}$ in the frequencies below $500 \mathrm{~Hz}$.

Figure 6.6. shows the major problem with the 2 level adaptation structure. For various driving conditions, excellent noise suppression takes place in the lower band while a slight noise enhancement takes place in the upper band. This phenomena occurs even though sub-band adaptation of the lower band is performed. This is believed to be caused by a bad step size $(\alpha(t))$ selection.

A tremendous amount of research needs to be done on the 2 level adaptation structure. If the step size selection problem could be resolved, this would be the best structure ever developed using the simple LMS algorithm. Meanwhile, the sequential structure has proven to being the best solution since it is always stable. 
Parallel Adaptation, Parallel Structure

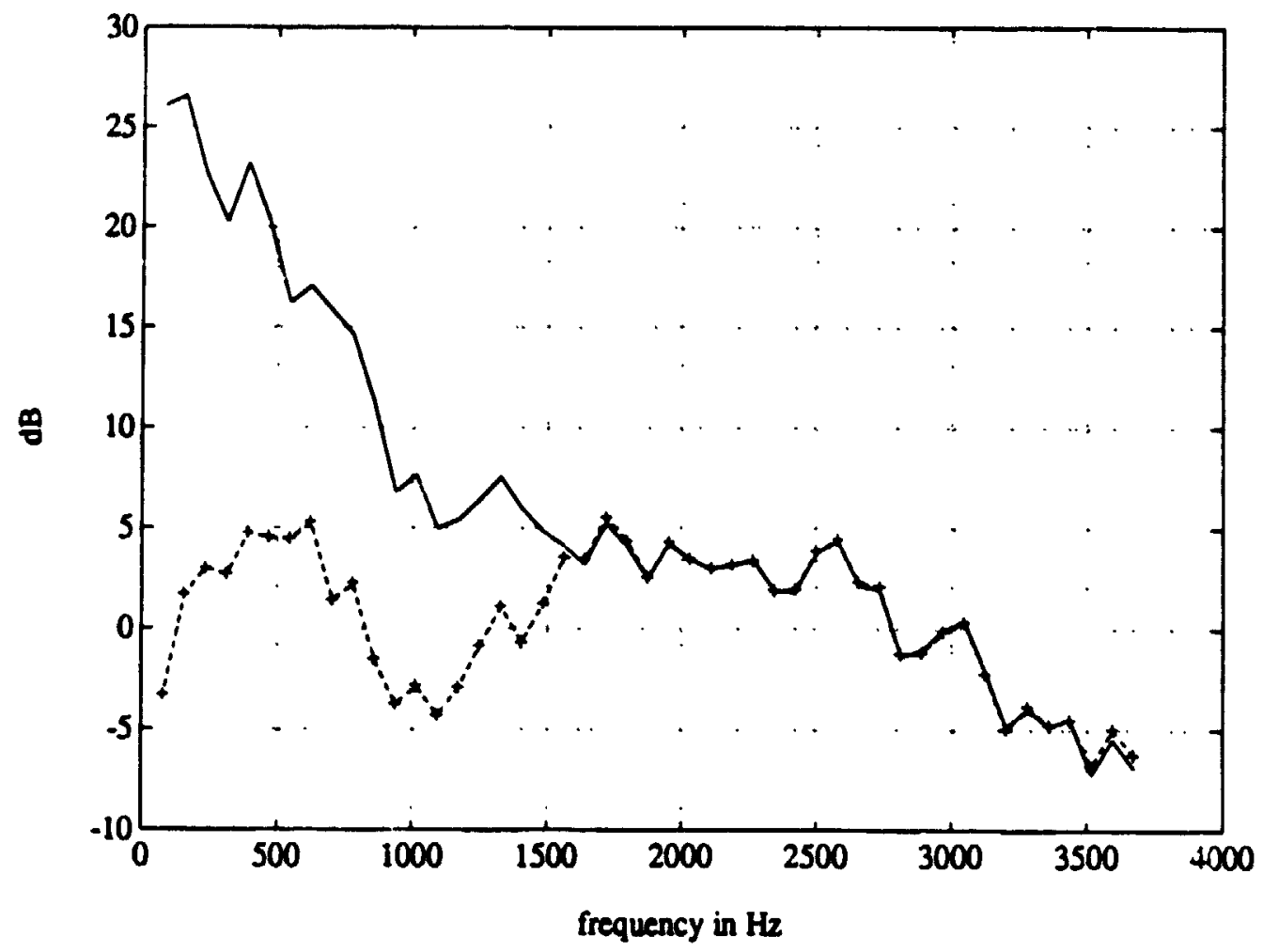

Figure 6.5 Result Of a 4 Microphone Sub-Band ANC (lower band filtering), Car Idling, Fan Off; Output :-t-

\subsubsection{Sequential Approach}

Figure 6.7. illustrates the concept of the sequential approach.

Each of the three reference outputs are fed in the three purt of a global filter $\underline{W}(t)$. The system equations become the same as for a 2 microphone ANC. The primary output estimation is given by

$$
\bar{x}(t)=\underline{y}^{T}(t) \underline{W}(t)
$$




\section{Parallel Adaptation, Parallel Structure}

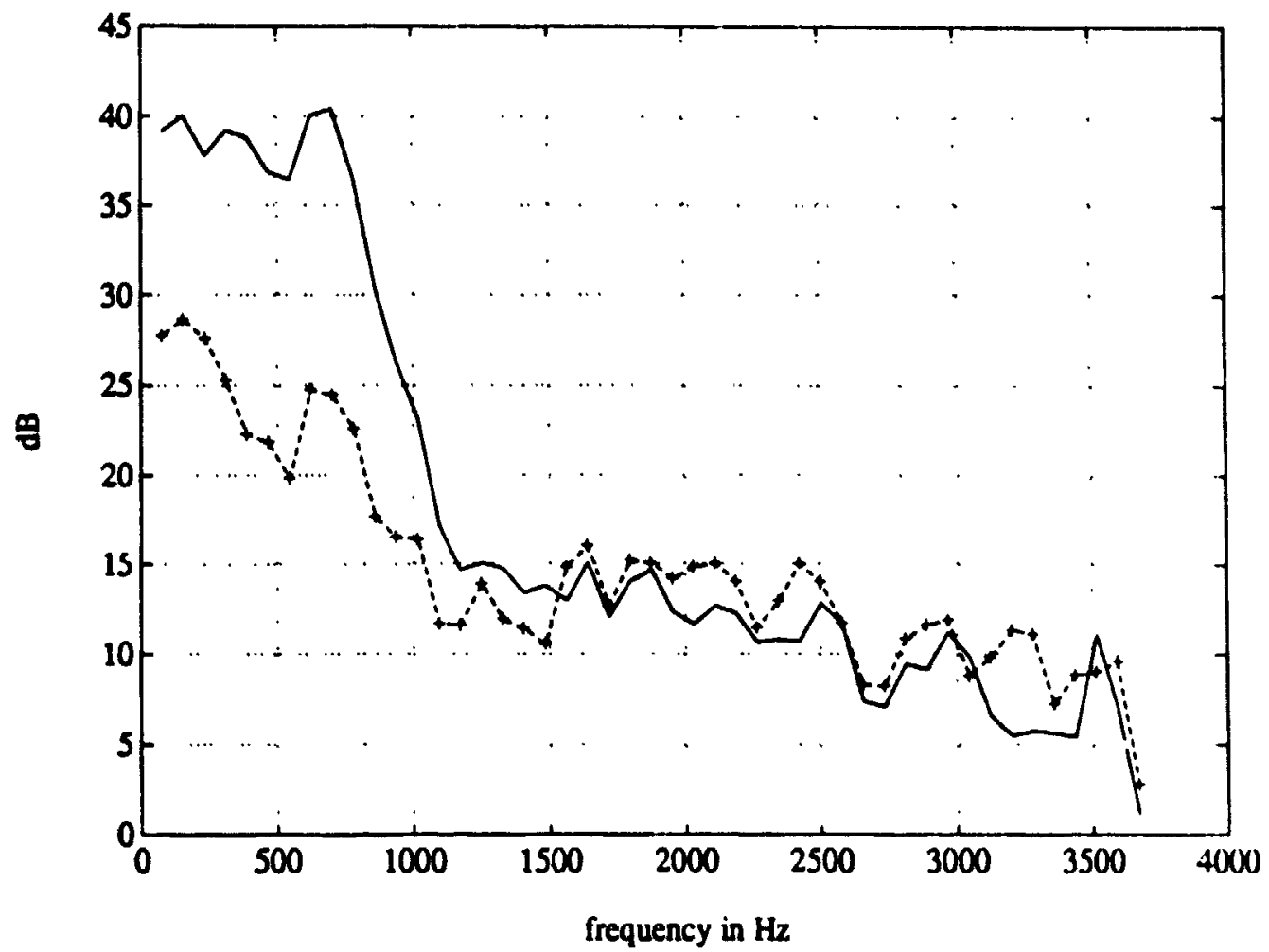

Figure 6.6 Result Of a 4 Microphone Sub-Band ANC (lower band filtering), Car Speed $100 \mathrm{Km} / \mathrm{hr}$, Fan Off; Output :-+where

$$
\underline{y}(t)=\left[\begin{array}{l}
\underline{y}_{1}(t) \\
\underline{y}_{2}(t) \\
\underline{y}_{3}(t)
\end{array}\right]
$$

and

$$
\underline{w}(t)=\left[\begin{array}{l}
\underline{w}_{1}(t) \\
\underline{w}_{2}(t) \\
\underline{w}_{3}(t)
\end{array}\right]
$$




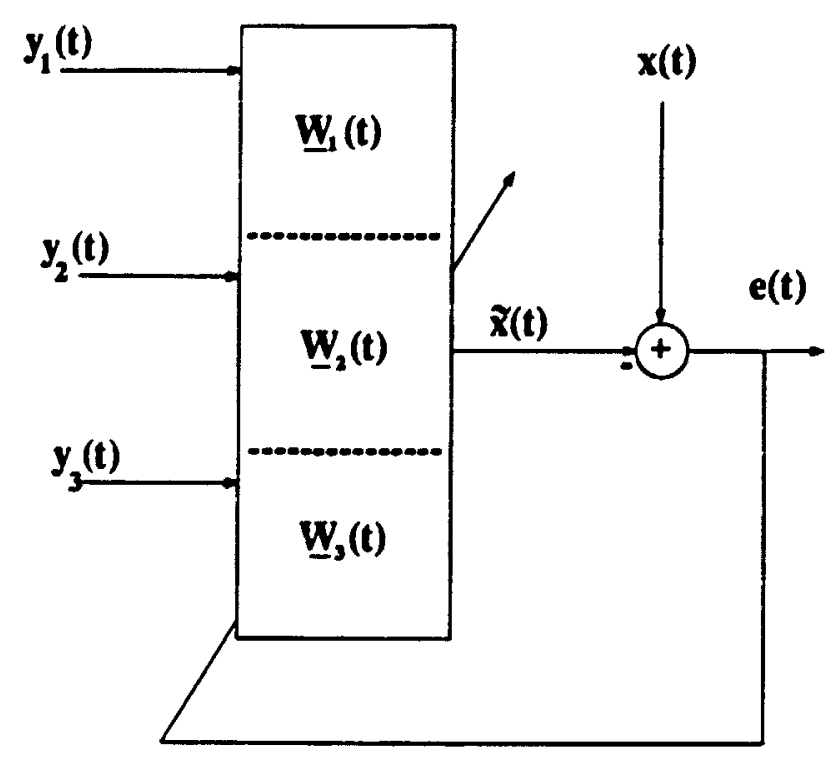

Figure 6.7 Parallel Adaptation, Sequential Structure

$y_{1}(t)$ is a $N \times 1$ vector composed of the last $N$ observations of the $i^{\text {th }}$ reference output. $W_{1}(t)$ is the $N \times 1$ FIR filter associated to the $i^{\text {th }}$ reference output.

Using equation (5.27), the coefficient are updated as such

$$
\underline{\mathbf{W}}(\mathbf{t}+\mathbf{1})=\underline{\mathbf{W}}(\mathbf{t})+\alpha(\mathbf{t}) \mathbf{e}(\mathbf{t}) \underline{\mathbf{y}}(\mathbf{t})
$$

where $\underline{W}(t+1)$ and $\underline{W}(t)$ are respectively the new and the old coefficients. $\alpha(t)$ is the step size. It is evaluated by

$$
\alpha(t)=\frac{a}{b+\sum_{i=1}^{3} \underline{y}_{i}^{T}(t) \underline{y}_{i}(t)}
$$

Evaluating $\alpha(t)$ with respect to the summation of all power level of each reference brings in a limiting factor. This is so since each reference power level is different from one another. The system task is very complex. It must perform temporal and spatial 
filtering simultaneously while gaging the adaptation to various power level. Obviously this does not seem optimum. Therefore, an in depth research should be done on the step size selection. Ideally it should be selected adaptively.

The error e(t) is simply the difference between the primary output $\mathbf{x}(t)$ and its estimate $\tilde{\mathbf{x}}(\mathbf{t})$. The variable $\mathbf{y}(t)$ wâs defined earlier.

This structure is very stable and provides in general, better noise suppression than the 2 microphone noise canceller. The main difference is that it makes use of the best noise cancellation feature of each location.

This structure is implemented using sub-band adaptation of the lower band. Figures 6.8 and 6.9 show that it provides good noise suppression in the lower band without affecting the upper band.

Comparing figures 6.5, 6.6 (parallel structure) with figures 6.8 and 6.9 (sequential structure), it is clear that the parallel structure performs better. However, due to it: step size selection, it sometimes lose its tracking capability. The sequential structure performs better than the 2 microphone ANC and never loses its tracking capability.

The two structures presented in this chapter prove that it is possible to perform excellent noise cancellation using a multiple reference microphone ANC. The system efficiency was about $12 \mathrm{~dB}$ for the sequential structure versus $15 \mathrm{~dB}$ for the parallel structure. The sequential approach prove itself to always be effective while the parallel structure sometimes loses its tracking capability. Further research must be carried through in order to fully define and understand the constraints surrounding the proposed structures. Also research toward an adaptive step size for the parallel structure should be investigated. 
Parallel Adaptation; Si:quential Structure

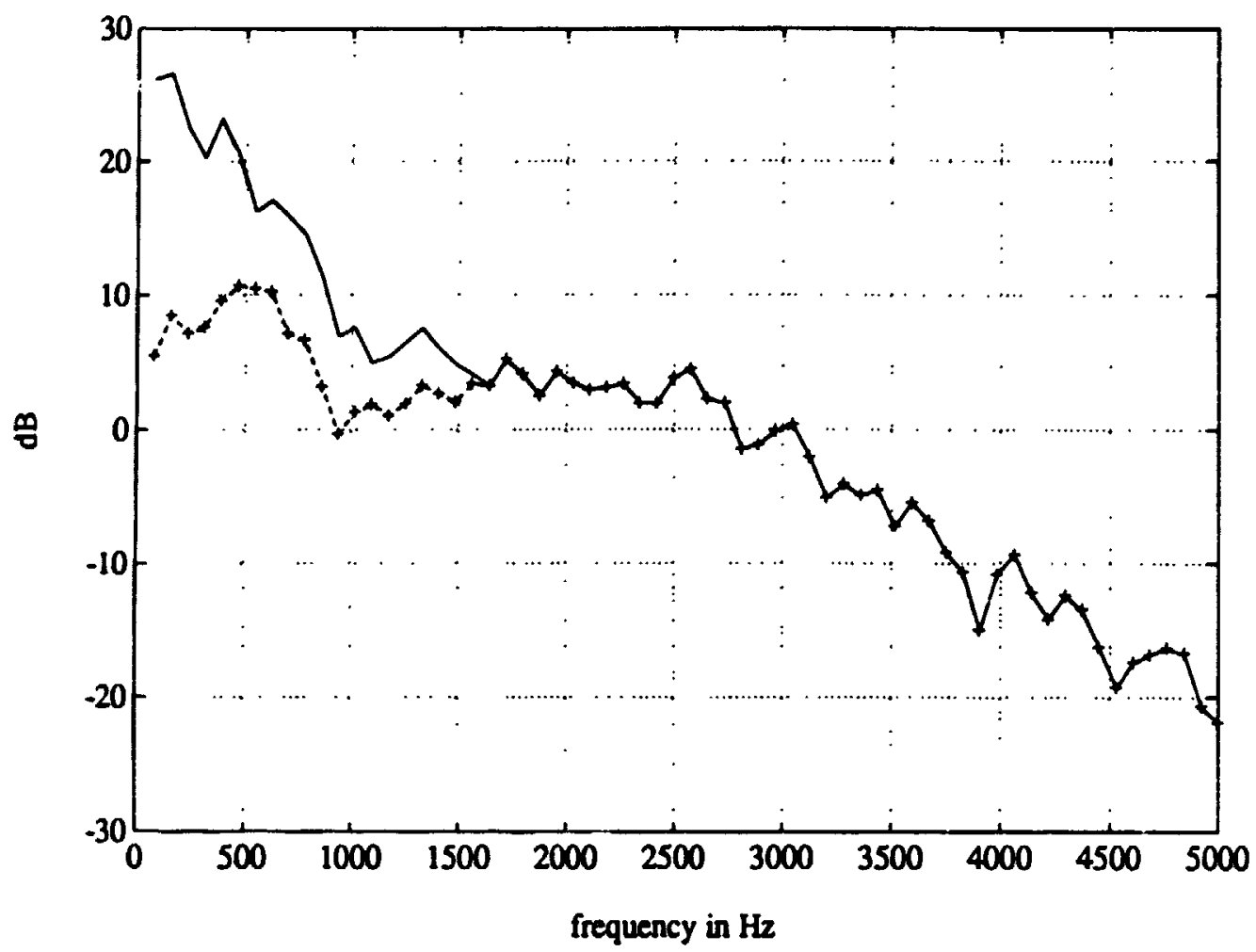

Figure 6.8 Result Of a 4 Microphone Sub-Band ANC (lower band filtering), Car Idling, Fan Off; Output :-+- 
Parallel Adaptation; Sequential Structure

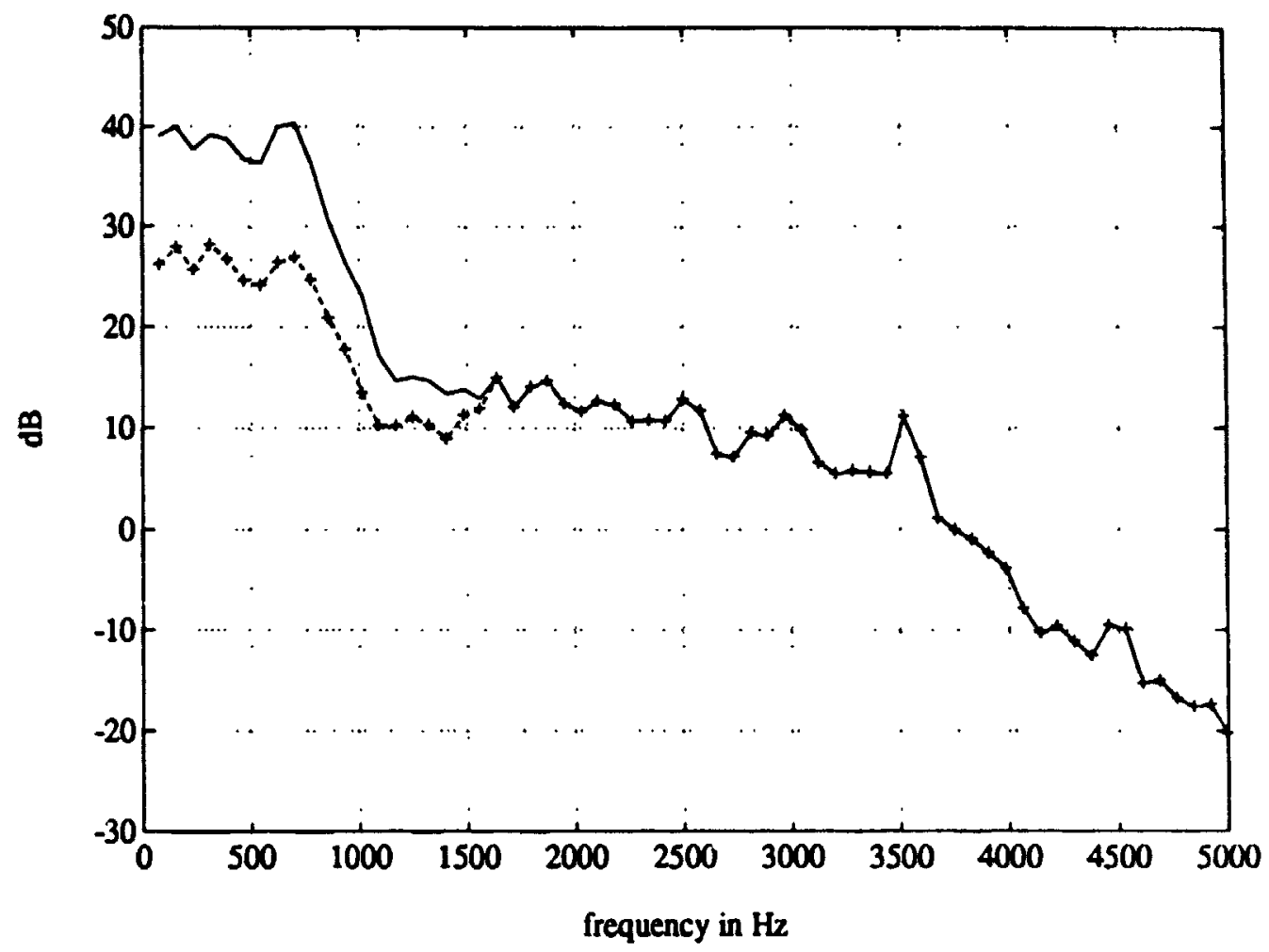

Figure 6.9 Result Of a 4 Microphone Sub-Band ANC (lower band filtering), Car Speed $100 \mathrm{Km} / \mathrm{hr}$, Fan Off; Output :-+- 


\section{Chapter 7 Conclusion}

\subsection{Discussion on the Results}

The thesis aim was to investigate the use of $\mathbf{2}$ or more microphones for cancelling the correlated background acoustic noise in medium size cars. An overview of existing background acoustic noise suppression techniques was presented.

The EM method based on the LMS algorithm was selected for testing the various ANC structures introduced throughout the thesis.

A qualitative noise model defining the interaction that exists between the multiple noise sources surrounding the normal use of a car with the various points of observation was developed. This constituted a first step toward completing the data analysis on real car data. It also helped in optimizing the various structure conceptions.

Using the noise model as system's insight, the data analysis was carried on real car data recorded on an 8 track tape recorder.

It was found that various noise processes associated to specific driving conditions had a particular spectral signature. The latter also depended on the point of observation in the car. Further more, it was found that the PSD could be divided in at least two sub-bands: the upper and the lower acoustic band. Because of the background acoustic noise complexity, the noise characteristics were fairly complex when analyzed in time domain. Hence the correlation could only be used in a qualitative manner. The time domain analysis performed on both sub-bands seemed to indicate that the upper band 
noise process differs from that observed in the lower band. This lead to developing a sub-band ANC.

A sub-band adaptation structure was successfully developed and tested on the car data. Background acoustic noise was achieved to a good level without enhancing the noise in any part of the acoustic band.

It was observed that under particular driving condition, some microphone locations provided a better reference source for noise suppression at specific frequencies. No microphone could prove itself as being the best one. It depended on both the driving condition and the microphone location.

Hence, there was a need for developing an algorithm which would control each microphone contribution with respect to the frequency band of interest. The problem was solved by introducing a two stage adaptation system. The first stage performed temporal filtering (frequency selection). The second performed spatial filtering (mic selection). Two primitive structures were presented, both of which offered concluding results.

Despite the fact that excellent results were obtained, there are still many unanswered question. For example, the mathematical model derived was based on a linear noise system. Recent researches seem to indicate that the background acoustic ri i.je characteristics are non-linear. Nevertheless, the system does react as the model predicted. This could well be due to the intuitive nature of the LMS algorithm. This is the object of further researches.

\subsection{Areas of Further Research}

As previously mentioned, it is a known fact that the background acoustic noise characteristics are rapidly changing. Throughout the thesis these were assumed constant 
for the period of interest. However, it is a factor worth researching. Further to it, the adaptive filter length could have been too long for allowing proper tracking of the system characteristics. I.E. the noise characteristics could vary too much for the adaptive filter response. Also, it is known that the LMS acts intuitively. Adding these factors seem to explain why the conventional ANC provides good noise suppression in a sub-band while yielding noise enhancement in the other band when noise suppression is performed over the entire acous. hand. These factors would also seem to indicate that there just enough time for adapting to the high power peak of the noise signal. Extensive research needs to be done on the matter in order to define an optimum adaptive filter length.

Also, the parallel ANC structures introduced provided good results. However, the better the results, less stable was the algorithm. Hence, research on algorithm stability should be carried on a large parallel structure variety.

Throughout the thesis, the adaptation step size were always set with respect to the noise power level. Various algorithms selecting the adaptation step size should be optimized.

Another area of research is the sub-band selection. I.E. in mobile telephony, the frequency band of interest ranges from about $150 \mathrm{~Hz}$ to $3500 \mathrm{~Hz}$. Adaptation should only be confined to this band. Also, the band could be split in more than two sub-band. This could however lead to a tone in band problem which can only be perceived by a listener. 


\section{References}

1. R.A. Goubran, H.M. Hafez, Background Acoustic Noise Reduction in Mobile Telephony", Proceedings of the $32^{\text {nd }}$ IEEE, Vehicular Technology Conference, Dallas, Texas, pp. 72-75, April 1987.

2. R.A. Goubran, R. Hebert and, H.M. Hafez, "Background Acoustic Noise Suppression Using Regressive Adaptive Filtering," Proceedings of the 36th IEEE Vehicular Technolgy Conference, Orlando, Florida, May 1990.

3. Meir Feder, Alan V. Oppenheim and Ehud Weinstein, "Maximum Likelihood Noise Cancellation Using the EM Algorithm," IEEE Transaction on Acoustic, Speech and, Signal Processing, vol. 37, no. 2, pp. 204-216, Feb 1989.

4. Bernard Widrow et al. "Adaptive Noise Cancelling: Principle and Applications," Proceedings of the IEEE, vol. 63, pp. 1692-1716, Dec 1975.

5. William A. Harrison, J. Lim, and E. Singer, "Adaptive Noise Cancellation in a Fighter Cockpit Environment," IEEE International Conference on Acoustic, Speech and, Signal Processing San Diego, California, pp. 18A.4.1-18A.4.4, April 1984. 
6. Steven F. Boll and D.C. Pulsipher, "Suppression of Acoustic Noise in Speech using Two Microphone Adaptive Noise Cancellation," IEEE Transaction on Acoustic, Speech and. Signal Processing, vol. 28, no. 6, pp. 752-766, Dec. 1980.

7. Svante Gunnarson and Lennart Ljung, "Frequency Domain Tracking Characteristics of Adaptive Algorithms," IEEE Transaction on Acoustic, Speech and, Signal Processing vol. 37, no. 7, pp. 1072-1089, July 1989.

8. Jayant an Noll, "Digital Coding of Waveform," Prentice-Hall, Inc. Englewood Cliffs, New Jersey 07632, Bell Telephone Laboratories, Incorporated, 1984.

9. A.P. French, "Vibration and Waves," Library of Congress, Catalog Card no. 6812181, pp. 209-216, Massachusetts Institute of Technology 1966.

10. Steven F. Boll, "Suppression of Acoustic Noise in Speech using Spectral Subtraction," IEEE Transaction on Acoustic, Speech and, Signal Processing, vol. 27, no. 2. pp. 113-120, April 1979.

11. Jae S. Lim, "Speech Enhancement," Prentice-Hall, Toronto, Canada, 1983.

12. R.D. Strum and D.E. Kirk, "First Principles of Discrete Systems and DSP," AddisonWesley Publishing Company, Don Mills Ontario, 1988.

13. Michael L. Honig, David G. Messerschmitt, "Adaptive Filters: Structure, Algorithms and Applications," Kluwer Academic Publishers, Bell Communication Research, Inc. 1984. 
14. S. Haykin, "Adaptive Filier Theory," Prentice Hall, Englewood Cliffs, New Jersey, 1986.

15. Mirchandani, Gaus and Bechtel, "Performance Characteristics of a Hardware Implementation of The Cross-Talk Adaptive Noise Canceller", IEEE, International Conference on Acoustic, Speech and, Signal Processing, Tokyo, Japan, pp. 93-96, April 86.

16. Pierre Comon and Dinh Tuan Pham, "An Error Bound For a Noise Canceller", IEEE Trans. on Acoustic, Speech and, Signal Processing, vol. 37, no. 10, pp. 1513-1517, Oct. 89.

17. Seiichi Nakamori, "On-Line Identification of Time Variant Parameters Using Covariance Information," IEEE International Conference on Acoustic, Speech and, Signal Processing, Tokyo, Japan, pp. 2723-2726, April 86.

18. Alan V. Oppenheim, "Applications of Digital Signal Processing," Englewood Cliffs, N.J. 07632, pp. 117-168, Prentice-Hall 1978.

19. Barry D. Van Veen, Kevin M Buckley, "Beamforming: A Versatile Approach to Spatial Filtering," IEEE Acoustic, Speech and, Signal Processing Magazine, pp. 4-24, April 1989.

20. W. Ambruster, R. Czarnach and P. Vary, "Adaptive Noise Cancellation With Reference Input-Possible Application And Theoretical Limits," Signal Processing III, EURASIP, pp. 391-394, 1986. 
21. W.P. Mikhael and P.D. Hill, "Acoustic Noise Cancellation in a Multiple Noise Source Environment," IEEE International Symposium on Circuits and Systems, Espoo Finland, pp. 2399-2402, April 1988.

22. I. Lecompte et al., "Car Noise Processing For Speech Input," IEEE, Conference on Acoustic, Speech and, Signal Processing, Glasgow, Scotland, pp. 512-5,5, May 1989.

23. John J. Shink, Bernard Widrow, "Bandpass Adaptive Pole-Zero Filtering," IEEE,Intemational Conference on Acoustic, Speech and, Signal Processing, Tokyo, Japan, pp. 2107-2110, April 1986.

24. Andre Gilloire, "Experiments With Sub-Band Acoustic Echo Cancellers For Teleconferencing," IEEE, International Conference on Acoustic, Speech and, Signal Processing, Dallas, Texas, pp. 2141-2144, April 1987.

25. W.P. Mikhael and P.D. Hill, "Performance Evaluation of a Real-Time TMS32010Based ANC," IEEE, International Conference on Acoustic, Speech and, Signal Processing, New York, New York, pp. 411-412, April 1988.

26. M.M. Goulding and J.S. Bird, "Speech Enhancement in Small Noisy Reverberant Enclosures," Canadian Conference on Electrical and Computer Engineering, Vancouver, Canada, pp. 219-222, 3-4 Nov 88.

27. H. Liang and N. Malik, "Reducing Cocktail Party Noise By Adaptive Array Filtering," Proceeding of the IEEE International Conference on Communications, Dallas, Texas, pp. 485-488, April 1987. 
28. A. Gilloire and M. Vetterli, "Adaptive Filtering in Sub-Bands," IEEE, International Conference on Acoustic, Speech and, Signal Processing, New York. New York. April 1988, pp.1572-1574.

29. W-Y Chan and D.D. Falconer, "Speech Detection For a Voice/Data Mobile Radio Terminal," Proceeding of the IEEE Intermational Conference on Communicaticns, Boston, MA., pp. 1650-1654, June 1983.

30. J.J. Shynk, R.P. Gooch anf B. Widrow," A Frequency-Domain Adaptive Pole-Zero Filter With Applications," IEEE, Intemational Conference on Acoustic, Speech and, Signal Processing, Tokyo, Japan, pp. 299-303, April 1986.

31. G.S. Kang and L.J. Fransen, "Experimentation Wi:h an Adaptive Noise-Cancellation Filter," IEEE, Trans. on Circuits and System, vol. CAS-34, no. 7, pp. 753-758, July 87.

32. P.M. Peterson, "Using Linearly-Constrained Adaptive Beamforming To Reduce Interference In Hearing Aids From Competing Talkers In Reverberant Rooms," IEEE, International Conference on Acoustic, Speech and, Signal Processing, Dallas, Texas, pp. 2364-2367, April 1987.

33. M. Feder et al. "Methods for Noise Cancellation Based on the EM Algorithm," IEEE, International Conference on Acoustic, Speech and, Signal Processing, Dallas, Texas, pp. 201-204, April 1987.

34. W.B. Mikhael and P.D. Hill , "Performance Evaluation Of A Real-Time TMS32010. 
Based Adaptive Noise Canceller," IEEE International Symposium on Circuits and Systems, San Jose, California, pp. 892-895, May 1986.

35. E. Andresdottir and R.W. Schafer, "Application of Adaptive Noise Cancelling in a Noisy Reverberant Environment," IEEE International Conference on Acoustic, Speech and, Signal Processing, Boston, Ma., pp. 57-60, April 1983.

36. C.P. Kwong, "Control-Theoretic Design of the LMS and the Sign Algorithms in Nonstationary Environments," IEEE Trans. on Acoustic, Speech and, Signal Processing, vol. 38, no. 2, pp. 253-259, Feb 1990.

37. G. Long et al., "Adaptive Transversal Filters With Delayed Coefficient Adaptation," IEEE, International Conference on Acoustic, Speech and, Signal Processing, Dallas, Texas, pp. 431-434, April 1987.

38. P. Darlington et al., "Adaptive Noise Reduction In Aircraft Communication Systems," IEEE, International Conference on Acoustic, Speech and, Signal Processing, Tampa, Fla., pp. 716-719, March 1985.

39. F. Ling, "Convergence Characteristics of LMS and LS Adaptive Algorithms For Signals With Rank-Deficient Correlation Matrices," IEEE, International Conference on Acoustic, Speech and, Signal Processing, New York, pp.1499-1502, New York, April 1988.

40. D.B. Roe, "Speech Recognition With A Noise-Adapting Codebook," IEEE, International Conference on Acoustic, Speech and, Signal Processing, Dallas, Texas, pp. 1139-1142, April 1987. 
41. W.B. Mikhael et al., "Optimum Adaptive Algorithms With Applications to Noise Cancellation," IEEE Trans. on Circuit and Systems,vol. CAS-31, no. 3, pp.312-315, March 84.

42. S. Jaggi and A.B. Martinez, "Upper and Lower Bounds of the Misadjustment in the LMS Algorithm," IEEE Trans. on Acoustic, Speech and, Signal Processing, vol. 38. no. 1, pp.164-166, Jan 1990.

43. W.A. Gardner and B.G. Agee, "Two-Stage Adaptive Noise Cancellation for Intermittent-Signal Applications," IEEE Trans. on Information Theory, Vol. 26, no. 6, pp. 746-750, Nov 1980.

44. D.W. Griffin and J.S. Lim, "Multiband Excitation Vocoder," IEEE Trans. on Acoustic, Speech and, Signal Processing Vol. 36, no. 8, pp. 1223-1235, Aug 88.

45. M.R. Sambur, "Adaptive Noise Canceling for Speech Signals," IEEE Trans. on Acoustic, Speech and, Signal Processing, vol. 26, no. 5, pp. 419-423, Oct 78.

46. J. Dunlop and M.J. Al-Kindi, "Applications of Adaptive Noise Cancelling To Diver Voice Communications," IEEE, International Conference on Acoustic, Speech and, Signal Processing, Dallas, Texas, pp. 1708-1711, April 1987.

47. R.R. Bitmead and B. Anderson, "Performance of Adaptive Estimation Algorithms in Dependent Random Environments," IEEE Trans. on Automatic Control, vol. AC. 25, no. 4, pp. 788-794, Aug 80. 
48. M. Bellanger and C.C. Evci, "Coefficient Wordlength Limitation In FLS Adaptive Filters," IEEE, International Conference on Acoustic, Speech and, Signal Processing, Tokyo, Japan, pp. 3011-3014, April 86.

49. D.H. Brandwood, "Noise-Space Projection:Music Without Eigenvectors," IEEE Proceeding, vol. 134, Pt. H, no. 3, pp. 303-309, June 87.

50. A. Sugiyama et al., "A Fast Convergence Algorithm For Adaptive FIR Filters," IEEE, International Conference on Acoustic, Speech and, Signal Processing, Glasgow, Scotland, pp. 892-895, May 1989.

51. C.P. Downing et al., "Improved Noise Canceller Performance By Means Of An Adaptive Arrangement Of IIR And FIR Filters," IEEE, International Conference on Acoustic, Speech and, Signal Processing, Glasgow, Scotland, pp. 2005-2008, May 1989.

52. M. Feder et al., "A New Class of Sequential and Adaptive Algorithms with Application to Noise Cancellation," IEEE, International Conference on Acoustic, Speech and, Signal Processing, New York, New York, pp. 557-560, April 1988.

53. P. Comon and J.L. Lacoume, "A Robust Adaptive Filter For Noise Reduction Problems," EEE, International Conference on Acoustic, Speech and, Signal Processing, Tokyo, Japan. pp. 2599-2602, April 86.

54. Y. Ariki et al., "Acoustic Noise Reduction By Two Dimensional Spectral Smoothing and Spectral Amplitude Transformation," IEEE, International Conference on Acoustic, Speech and, Signal Processing, Tokyo, Japan, pp. 97-100, April 86. 
55. M.G. Amin, "Adaptive Noise Cancelling in the Spectrum Domain," IEEE, Intermational Conference on Acoustic, Speech and, Signal Processing, Tokyo, Japan, pp. 2959-2962, April 86.

56. G. Faucon et al., "Study and Comparison of Three Structures For Enhancement of Noisy Speech," IEEE, International Conference on Acoustic, Speech and, Signal Processing, Glasgow, Scotland, pp. 385-388, May 1989.

57. M. Feder and E. Weinstein, "Parameter Estimation of Superimposed Signals Using the EM Algorithm," IEEE Trans. on Acoustic, Speech and, Signal Processing vol. 36, no. 4, pp. 477-489, April 88.

58. F. Mosteller and J.W. Tukey, "Data Analysis and Regression," Addison-Wesley Publishing Company, Don Mills, Ontario, 1977.

59. R.H. Myers, "Classical and Modern Regression With Applications," Duxbury Press, Boston, Ma., 1986.

60. M.H. Pesaron and L.J. Slater, "Dynamic Regression: Theory and Algorithms". Halsted Press, Toronto, Ontario, 1980.

61. N. Kerlinger and E. Pedhazur, "Multiple Regression in Behavior Research," Holt, Rinehart and Winston Inc., Montreal 1973.

62. W.A. Harrison, "Speech Enhancement Using Multiple Microphones," MIT Lincoln Laboratory, Technical Report 691, 15 Nov 1984. 
63. Y.H. Lee et al., "Efficient Impulsive Noise Suppression Via Nonlinear Recursive Filtering," IEEE Trans. on Acoustic, Speech and, Signal Processing, vol. 37, no. 2. pp. 317-319, Feb 89.

64. R.P. Ramachandran and P. Kabal, "Pitch Prediction Filters in Speech Coding," IEEE Trans. on Acoustic, Speech and, Signal Processing, yol. 37, no. 4, pp. 99-105,April 89.

65. J.B. Burl, "Estimating the Basis Functions of the Karhunen-Love Transform," IEEE Trans. on Acoustic, Speech and, Signal Processing, vol. 37, no. 1, pp. 107-108, Jan 89.

66. M. Shensa, "Performance of the Adaptive Noise Canceller with a Noisy Reference - Non-Wiener Solutions," Technical Report 381, Naval Ocean Systems Center, San Diego California, 92152, 15 March 1979.

67. Friedland, "Control System Design, An Introduction to State-Space Methods," McGraw-Hill Book Company, Montreal, 1986.

68. R.C. Gonzalez and P. Wintz, "Digital Image Processing," Addison-Wesley Publishing Company, 2nd Edition, Don Mills, Ontario, 1987.

69. R. Hebert, R.A. Goubran and, H.M. Hafez, "Analysis of Background Acoustic Noise in Cars," Canadian Conference on Electrical and Computer Engineering, Ottawa, Ontario, Canada, 4-6 Septemter 1990. 
70. Ronald A. Belaire, "Noise Cancellation Using a Reference Noise Signal," 4th Year Project, Department of Systems and Computer Engineering, Carleton University, Ottawa, Canada, K1S 5B6, April 7, 1989 

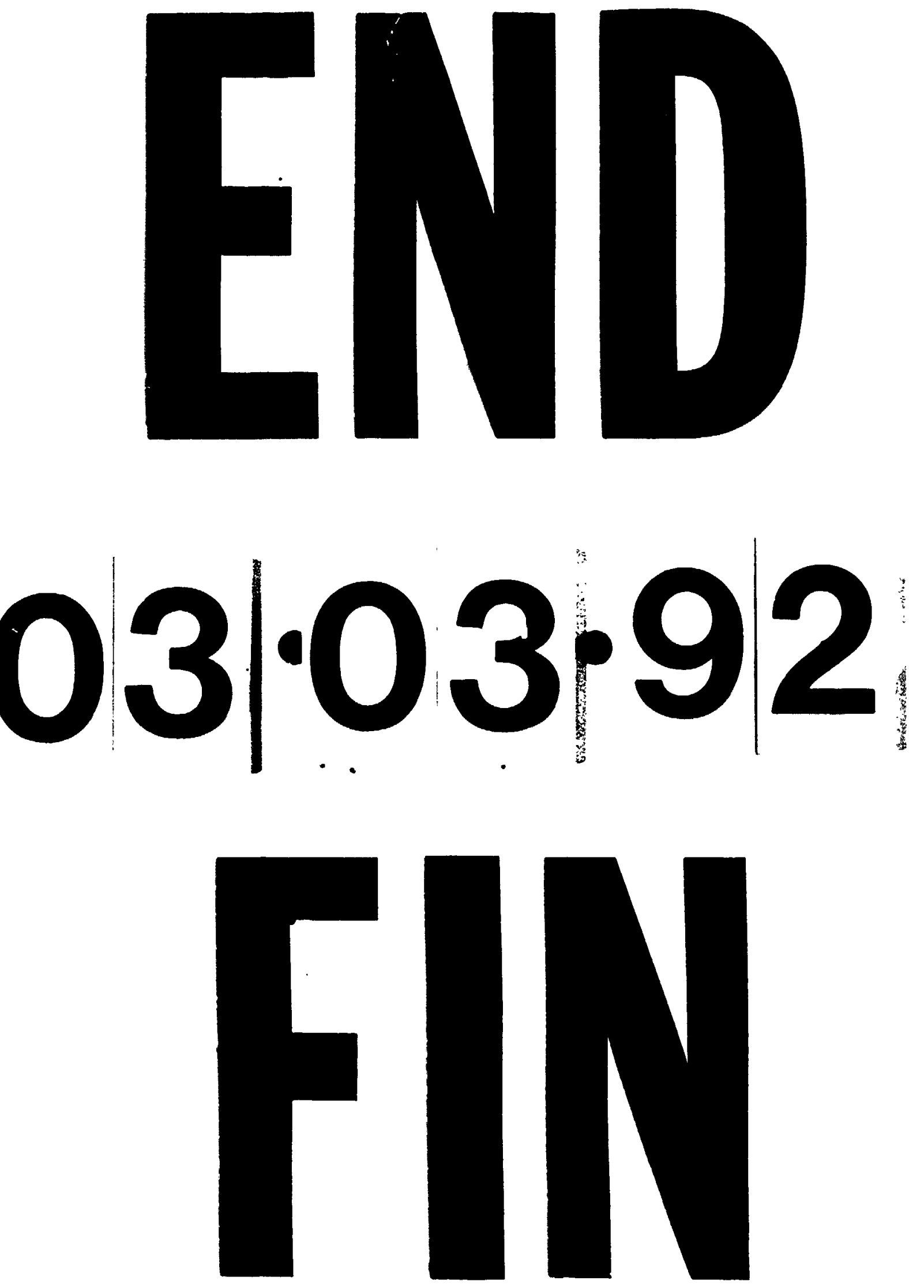